\title{
Molecular gas in the Galactic center region
}

\section{Data from a large scale $\mathrm{C}^{18} \mathrm{O}(J=1 \rightarrow 0)$ survey}

G. Dahmen ${ }^{1,4}$, S. Hüttemeister ${ }^{2,1,5}$, T.L. Wilson ${ }^{1}$, R. Mauersberger ${ }^{6,1}$, A. Linhart ${ }^{1}$, L. Bronfman ${ }^{3}$, A.R. Tieftrunk ${ }^{1,7}$, K. Meyer ${ }^{1}$, W. Wiedenhöver ${ }^{1}$, T.M. Dame ${ }^{2}$, E.S. Palmer ${ }^{2}$, J. May ${ }^{3}$, J. Aparici ${ }^{3}$, and F. Mac-Auliffe $\mathrm{e}^{3,7}$

1 Max-Planck-Institut für Radioastronomie, Auf dem Hügel 69, 53121 Bonn, Germany

2 Harvard-Smithsonian Center for Astrophysics, 60 Garden Street, Cambridge, MA 02138, U.S.A.

3 Departamento de Astronomía, Universidad de Chile, Casilla 36-D, Santiago de Chile, Chile

4 Physics Department, Queen Mary \& Westfield College, University of London, Mile End Road, London E1 4NS, UK

${ }^{5}$ Radioastronomisches Institut, Universität Bonn, Auf dem Hügel 71, 53121 Bonn, Germany

${ }^{6}$ Steward Observatory, University of Arizona, Tucson, AZ 85721, U.S.A.

7 Swedish-ESO Submillimetre Telescope, European Southern Observatory, Casilla 19001, Santiago de Chile 19, Chile

Received July 1, 1996; accepted February 24, 1997

\begin{abstract}
A large scale survey of the Galactic center region in the $\mathrm{C}^{18} \mathrm{O}(J=1 \rightarrow 0)$ transition is presented. This survey was obtained with the $1.2 \mathrm{~m}$ Southern Millimeter-Wave Telescope (SMWT) at the Cerro Tololo Interamerican Observatory (CTIO) near La Serena, Chile. It covers the region $-1.05 \leq l \leq+3.6$ and $-0.9 \leq b \leq$ +0.75 with a grid spacing of $9^{\prime}$, i.e. the sampling is at full FWHP beamwidth. 357 positions were in total observed. After reviewing the instrumentation of the $1.2 \mathrm{~m}$ SMWT, the observing techniques, and the methods used in the data reduction, the data of the survey are presented and morphologically described. In addition, data of the $\operatorname{HNCO}\left(5_{0,5}-4_{0,4}\right)$ line are presented, which was also included in the large bandwidth of the spectrometer. ${ }^{12} \mathrm{CO}(1-0)$ measurements performed for comparison purposes are presented and compared with other ${ }^{12} \mathrm{CO}$ results. The maps of the $\mathrm{C}^{18} \mathrm{O}(1-0)$ survey demonstrate that there are great differences between the distribution of the optically thin $\mathrm{C}^{18} \mathrm{O}(1-0)$ emission and the usually optically thick ${ }^{12} \mathrm{CO}(1-0)$ emission.
\end{abstract}

Key words: telescopes — surveys — ISM: molecules, structure - Galaxy: center — radio lines: ISM

\section{Introduction}

The molecular gas in the central region of our Galaxy has been studied often and extensively on several scales.

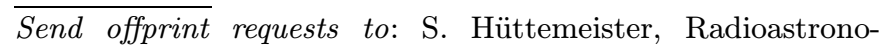
misches Institut, Universität Bonn, Germany.
However, on the scale of the complete central region or even on the scale of a few degrees only very few molecular transitions have been mapped; the large extent of the Galactic center region makes such surveys very time-consuming. So far, relatively complete maps exist in ${ }^{12} \mathrm{CO}(1-0)$ (e.g. Bitran 1987/Bitran et al. 1997; Jackson et al. 1996; Uchida et al. 1997), ${ }^{13} \mathrm{CO}(1-0)$ (e.g. Heiligman 1987; Bally et al. 1987, 1988), $\operatorname{HCN}(1-0)$ (Jackson et al. 1996), and ${ }^{12} \mathrm{CO}(2-1)$ (Oka et al. 1996). There are also maps covering at least a few degrees in the $\mathrm{OH} 1665$ - and $1667-\mathrm{MHz}$ ground state lines (Cohen \& Few 1976; Cohen \& Dent 1983), in $\mathrm{H}_{2} \mathrm{CO}\left(1_{1,1}-1_{1,0}\right)$ (Whiteoak \& Gardner 1979; Zylka et al. 1992), and in CS(2 - 1) (Bally et al. $1987,1988)$. It is, of course, desirable to obtain more information on the distribution and abundance of other important molecules as tracers of physical properties toward the central region of the Milky Way. Since the $J=1 \rightarrow 0$ or $2 \rightarrow 1$ transition of even ${ }^{13} \mathrm{CO}$ is in many cases saturated, it is interesting to compare the distribution of the $J=1 \rightarrow 0$ transition of ${ }^{12} \mathrm{CO}^{1}$, which many studies have assumed to be always optically thick, with that of the (at least on large scales, and in most cases also on small scales) optically thin $\mathrm{C}^{18} \mathrm{O}(J=1 \rightarrow 0)$ line.

In this work, we present a large scale survey of the Galactic center region in the $J=1 \rightarrow 0$ transition of $\mathrm{C}^{18} \mathrm{O}$ at $109.782160 \mathrm{GHz}$ (Lovas 1992). The survey was carried out using the $1.2 \mathrm{~m}$ Southern Millimeter-Wave Telescope (SMWT) at the Cerro Tololo Interamerican Observatory

1 Following the typical astronomical convention, the atomic weight of the main isotopes will not be given explicitly, e.g., ${ }^{13} \mathrm{CO}$ and $\mathrm{C}^{18} \mathrm{O}$. However, to better distinguish different carbon monoxide isotopomers we will denote $\mathrm{CO}$ as ${ }^{12} \mathrm{CO}$. 
(CTIO) near La Serena, Chile. This telescope was built for large scale surveys of the southern sky, especially of the Galactic center region. The receiver of the telescope was optimized for carbon monoxide observations at $3 \mathrm{~mm}$ wavelength and has a tuning range from $109 \mathrm{GHz}$ to $120 \mathrm{GHz}$. In this frequency range, the telescope has a beamsize of about $9^{\prime}$ and hence is the appropriate instrument for large scale surveys. The SMWT and its northern twin telescope, the $1.2 \mathrm{~m}$ Northern Millimeter-Wave Telescope (NMWT), now at the Harvard-Smithsonian Center for Astrophysics (CfA) in Cambridge (Mass.), U.S.A., have been used to map the complete Galactic plane and several other regions of interest in ${ }^{12} \mathrm{CO}(1-0)$ (Dame et al. 1987).

The survey presented here constitutes the first major observing project in the rare isotopomer $\mathrm{C}^{18} \mathrm{O}$ to be carried out by one of the $1.2 \mathrm{~m}$ Millimeter-Wave Telescopes (MWTs). It covers the range from $l=-1.05$ to +3.6 in Galactic longitude and the range from $b=-0.9$ to +0.75 in Galactic latitude. In total, 357 positions were observed. The final spectra at each position consist of an average of 12 or more individual spectra, altogether more than 6500 spectra were taken. Preliminary presentations of the data are given in Dahmen et al. (1993), Linhart (1994), and Mauersberger (1994), a first glance of the complete data is presented in Dahmen et al. (1996). Because of the large bandwidth of the backend, the $5_{0,5}-4_{0,4}$ transition of isocyanic acid (HNCO) at $109.905573 \mathrm{GHz}$ (Lovas 1992) could be observed simultaneously with the $\mathrm{C}^{18} \mathrm{O}(1-0)$ line as long as the emission did not appear at too negative velocities. In addition, ${ }^{12} \mathrm{CO}(1-0)$ observations were performed to directly compare the ${ }^{12} \mathrm{CO}$ data obtained with the $1.2 \mathrm{~m}$ SMWT in the configuration used to other ${ }^{12} \mathrm{CO}$ data and, thus, check the comparability and calibration of the data presented here.

In Sect. 2, the instrumentation of the $1.2 \mathrm{~m}$ SMWT is outlined. In Sect. 3, the observations and data reduction are described. In Sect. 4 , the results of the $\mathrm{C}^{18} \mathrm{O}(1-0)$ survey are presented. These consist of the full set of survey spectra, spatial maps of integrated intensities, longitudevelocity plots, and latitude-velocity plots. The section also includes a morphological description of the features mapped. In Sect. 5, the $\operatorname{HNCO}\left(5_{0,5}-4_{0,4}\right)$ data are presented and described in the same way as the $\mathrm{C}^{18} \mathrm{O}$ data. In Sect. 6 , the ${ }^{12} \mathrm{CO}$ data are shown and compared to other ${ }^{12} \mathrm{CO}(1-0)$ measurements. Finally, in Sect. 7, preliminary conclusions are drawn from the morphological information presented so far. A detailed analysis and discussion of the data will be given in a follow-up paper (Paper II, Dahmen et al. 1997).

\section{The Instrumentation of the $1.2 \mathrm{~m}$ SMWT}

\subsection{The basic telescope system}

The $1.2 \mathrm{~m}$ SMWT was built in the period from 1980 to 1983 as the southern twin of the $1.2 \mathrm{~m} \mathrm{NMWT}$, then at
Columbia University, New York City, U.S.A. The SMWT started operation at CTIO at the beginning of 1983 (Cohen 1983). The antenna is a fast Cassegrain reflector with a 1.2 meter parabolic primary and a $18.8 \mathrm{~cm}$ hyperbolic secondary (Grabelsky et al. 1987). The effective f/D of the telescope is 3.79 , and the surface accuracy of the primary is $20 \mu \mathrm{m}$, which is better than $\lambda / 100$ at the wavelength of the $J=1 \rightarrow 0$ line of ${ }^{12} \mathrm{CO}, \lambda^{12} \mathrm{CO}=2.6 \mathrm{~mm}$ (Cohen 1983). The primary is made from a single aluminium casting, and the complete telescope is housed in an astrodome whose slit is completely covered by a thin screen of Griffolyn, a polyolefin fabric almost totally transparent at $2.6 \mathrm{~mm}$. The mount of the telescope can move by $5^{\circ}$ in less than $1 \mathrm{~s}$, and hence for position switching one can use reference locations degrees away from the source position in switching cycles of only $30 \mathrm{~s}$. This allows rapid position switching (Bronfman et al. 1988).

The telescope has a superheterodyne receiver, which is tunable from $109 \mathrm{GHz}$ to $120 \mathrm{GHz}$. Its first stage is cooled to $77 \mathrm{~K}$ by liquid nitrogen and consists of a resonant ring LO diplexer with a signal loss of $0.2 \mathrm{~dB}$, a double-sideband Schottky barrier diode mixer with a noise temperature of $110 \mathrm{~K}$ and a conversion loss of $5.2 \mathrm{~dB}$, an impedance matching transformer, and a GaAs field-effect-transistor (FET) amplifier operating at the first intermediate frequency (IF) of $1390 \mathrm{MHz}$. The amplifier has a noise temperature of $15 \mathrm{~K}$ and a gain of $\sim 30 \mathrm{~dB}$, constant to $1 \mathrm{~dB}$ over a bandwidth of $150 \mathrm{MHz}$. The second stage, at ambient temperature, consists of standard commercial components which further amplify the IF signal and convert it to the second IF of $150 \mathrm{MHz}$ (Bronfman et al. 1988, 1989).

The full width at half-maximum (FWHM) of the main beam of the telescope was measured to be 8.8 at 115.3 GHz, with sidelobes more than $18 \mathrm{~dB}$ below the main beam (Bronfman et al. 1988, 1989).

The spectrometer permanently installed at the telescope is a 256 channel filterbank with a resolution of $100 \mathrm{kHz}$ (Palmer 1984). Thus, the total bandwidth of this backend is $25.6 \mathrm{MHz}$. This corresponds to only $69.9 \mathrm{~km} \mathrm{~s}^{-1}$ at $109.8 \mathrm{GHz}$, the $\mathrm{C}^{18} \mathrm{O}(1-0)$ frequency. This small bandwidth is insufficient for Galactic center observations since the $\mathrm{CO}$ emission is known from previous surveys to cover the velocity range from -250 to $+300 \mathrm{~km} \mathrm{~s}^{-1}$ (see e.g. Bania 1977, 1980, 1986 for ${ }^{12} \mathrm{CO}$, or Bally et al. 1987, 1988 for $\left.{ }^{13} \mathrm{CO}\right)$.

\subsection{The system upgrade of the MPIfR in 1993}

Before the $\mathrm{C}^{18} \mathrm{O}(1-0)$ survey could be started, the telescope control software and hardware had to be improved, a broadband backend installed, and data reduction facilities established.

First, a computer system based on an Apple Macintosh was installed at the SMWT. This allowed to run the control software of the NMWT after adjusting it to the Cerro Tololo site. 
The Max-Planck-Institut für Radioastronomie (MPIfR) in Bonn, Germany, made available a broadband (total bandwidth $=795 \mathrm{MHz}$ ) acousto-optical spectrometer, hereafter AOS, (see Linhart 1994 for a detailed description of the AOS). The optical system of the AOS laser is designed to illuminate 1499 pixels of the charge coupled device (CCD). Each pixel has a frequency separation of $0.536695 \mathrm{MHz}$. The frequency resolution of the dispersion of the laser due to the acoustical signal is $0.78 \mathrm{MHz}$.

For the use at the $1.2 \mathrm{~m}$ SMWT, the AOS had to be modified in a number of ways to be compatible with the existing system (see Linhart 1994 for details). One requirement of the system affected the observations significantly: Because the telescope control software was designed to work with 256 channel backends, the AOS had to be adjusted to read out only 512 of the 1499 channels; furthermore, 2 AOS channels were combined to produce 1 channel. Thus, the AOS became a 256 channel backend with a frequency separation of $1.07339 \mathrm{MHz}$ per channel. This corresponds to $2.93 \mathrm{~km} \mathrm{~s}^{-1}$ at the $\mathrm{C}^{18} \mathrm{O}(1-0)$ frequency and a total bandwidth of $274.8 \mathrm{MHz}\left(=750 \mathrm{~km} \mathrm{~s}^{-1}\right)$. This nearly completely utilized the total receiver bandwidth of $300 \mathrm{MHz}$. It covered the total velocity range of CO emission in the Galactic center region and, in addition, allowed for enough range in velocity to accurately determine baselines. The resolution was adequate for accurate measurements of the wide lines common in the Galactic center region.

On-site data reduction, essential to ensure homogenous, high quality data, was made possible by the installation of a separate workstation, and the implementation of the Grenoble Image and Line Data Analysis System (GILDAS) software package. A dedicated program package, called CTOL, for data transfer via serial link and translation to the format used by the Continuum and Line Analysis Single-dish Software (CLASS) which is part of GILDAS was developed (see Dahmen 1995 for details).

The complete system including all upgrades is illustrated in Fig. 1.

\section{Observations and data reduction}

The observations were carried out between the beginning of August 1993 and the end of August 1994. On Cerro Tololo, the Galactic center is above an elevation of $20^{\circ}$ for 11 hours per day. Because the telescope cannot track sources at elevations higher than $78^{\circ}$, the total daily observing time was 9 hours.

The $\mathrm{C}^{18} \mathrm{O}(1-0)$ line was placed in the lower sideband of the receiver because the line frequency is close to the lower limit of the tuning range. With this, the image frequency of the system was $112.556165 \mathrm{GHz}$. The doublesideband receiver temperature, $T_{\text {rec }}$, determined with a standard "Hot-Cold-Test" using the filterbank backend, was always in the range of 180 to $220 \mathrm{~K}$. The radial veloc-

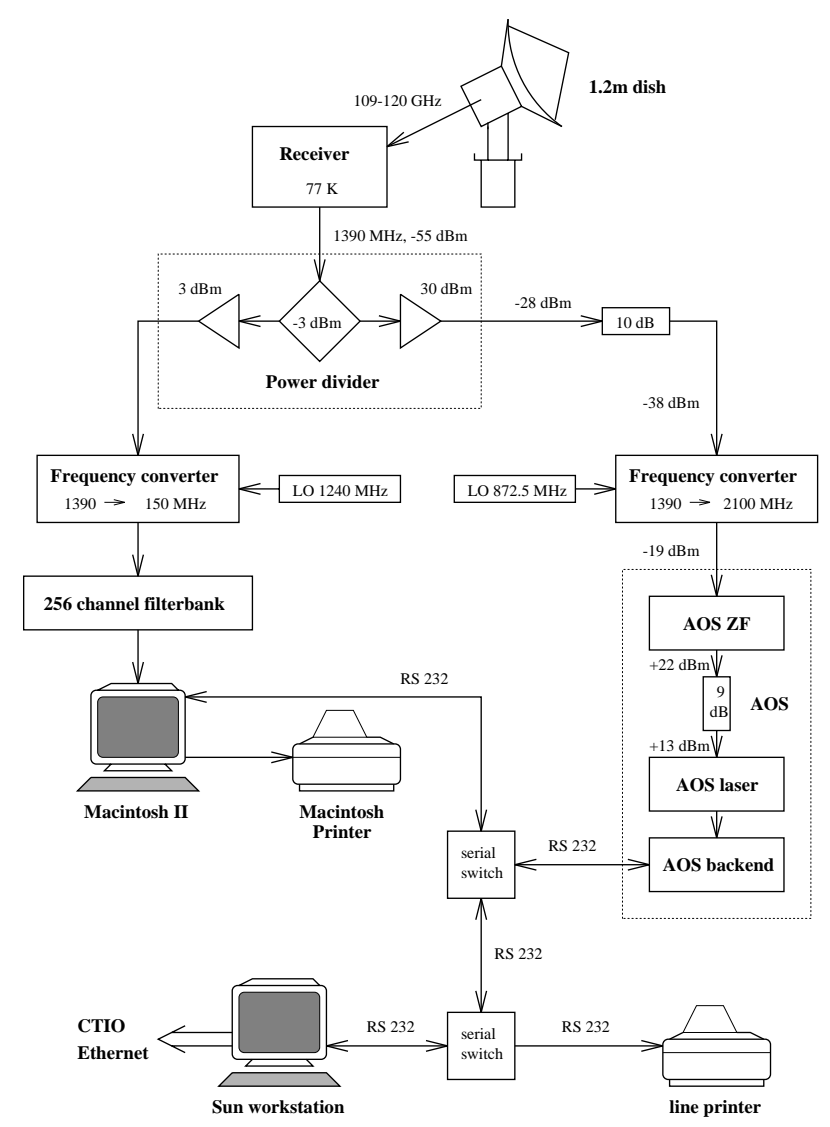

Fig. 1. The complete system of the $1.2 \mathrm{~m}$ SMWT on Cerro Tololo (CTIO) for the $\mathrm{C}^{18} \mathrm{O}(1-0)$ Galactic Center Survey. The receiver signal was split into the branch for the filterbank and the branch for the AOS. The Macintosh controls the telescope drive, the frequency synthesizer, the filterbank, and the AOS. It is connected to the Sun workstation for data reduction via the serial link

ity of the center channel of the AOS was set to $0 \mathrm{~km} \mathrm{~s}^{-1}$ with respect to the Local Standard of Rest (LSR).

A detailed description of the observing procedure is given in Appendix A. This includes the scan integration with weighted-OFFs position switching, the methods of scan calibration, and the telescope pointing.

\subsection{Survey plan}

Because the FWHM beam width of the telescope is 9.2 at the $\mathrm{C}^{18} \mathrm{O}(1-0)$ line frequency, a fully sampled map requires data spaced at 4.6 . As a compromise to cover a larger region of the Galactic center, we have used a grid spacing of $9^{\prime}(=0.15)$, that is half-sampling. We chose the grid axes to follow Galactic coordinates centered on $\left(l, b=0^{\circ}, 0^{\circ}\right)$. Since the $\mathrm{C}^{18} \mathrm{O}$ emission is weak and the AOS was available only for a limited time, we decided to concentrate on the inner region of the Galactic center 
and to extend the map only to the Clump 2 region (Bania 1977 ) in the range of $l=+3.0$ to +3.5 .

\subsection{Data acquisition}

Each position was observed with a series of 10-minutescans. Typically, a single scan had an rms noise temperature per channel of about 0.12 to $0.15 \mathrm{~K}$, with extremes from 0.08 to $0.25 \mathrm{~K}$, on a $T_{\mathrm{A}}^{*}$ scale, as obtained with the AOS (see Sect. 3.4.2). The scans were averaged until the rms noise temperature per channel in the average spectrum was less than $0.05 \mathrm{~K}$. Typically, twelve 10-minutescans per position were needed to reach this limit, and on average $0.04 \mathrm{~K}$ was reached. Thus, two to three positions per day could be completely measured.

This long total integration time was necessary to reach a reasonable signal-to-noise ratio in the spectra. The most intense $\mathrm{C}^{18} \mathrm{O}(1-0)$ emission, at $\mathrm{Sgr} \mathrm{B} 2$, is only $0.45 \mathrm{~K}$, i.e. for this source, the maximum signal-to-noise ratio per channel was about 10. Away from this peak the emission decreases quickly to about $0.2 \mathrm{~K}$ peak temperature and outside the inner region to below the $3 \sigma$ detection limit of $0.15 \mathrm{~K}$. At many grid positions $\mathrm{C}^{18} \mathrm{O}(1-0)$ emission is only detected by integrating over a number of velocity channels.

The OFF positions used, verified to be free of ${ }^{12} \mathrm{CO}(1-0)$ emission stronger than $0.04 \mathrm{~K}$, are given in Appendix B. Since these OFF positions are appropriate for ${ }^{12} \mathrm{CO}(1-0)$ observations, they are very well suited for measurements of the much weaker $\mathrm{C}^{18} \mathrm{O}(1-0)$ emission.

\subsection{Data reduction}

Each spectrum was examined individually, and bad spectrometer channels, difficult to avoid on a CCD chip with 1499 channels, were removed and replaced by interpolating the neighbouring channels. After this cleaning, all spectra of the same position were averaged, the regions for the baseline subtraction were selected and a polynomial baseline was subtracted. By inspecting every single spectrum individually we checked for artefacts which might mimic emission in the averaged spectrum. Such features occur rarely. These were not considered to be emission but rather as instrumental baseline effects contributing to the uncertainties.

The reduction procedure was as follows: If the noise in all spectra was roughly the same, as was the case for most positions, the spectra were first averaged, weighted by time, i.e. equally. Then, the regions for the baseline subtraction were selected. The average spectrum was used for this selection, because here weak line emission could be recognized more easily and, therefore, the window regions, where the channel content could not be used for the baseline fit, could be set accordingly. In cases of uncertainty these windows were determined by comparison with ${ }^{12} \mathrm{CO}(1-0)$ spectra. If the noise of individual spectra

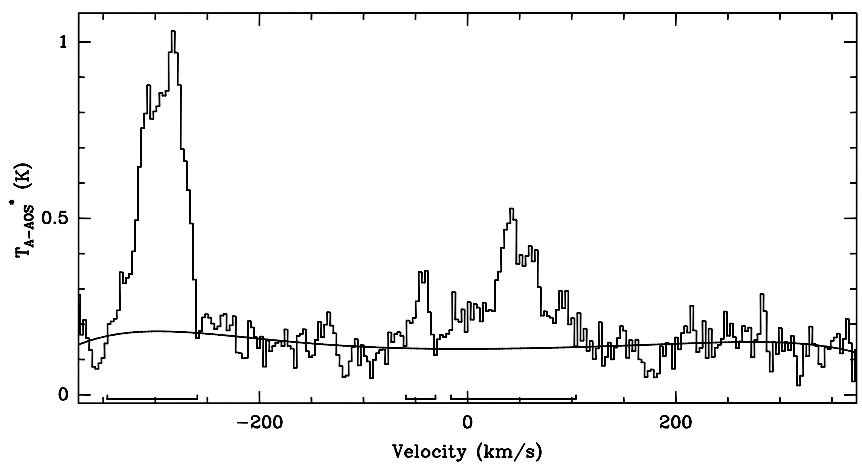

Fig. 2. The averaged spectrum of 13 individual 10-minute-spectra toward the position $l=0.75, b=-0^{\circ} .15$ of the $\mathrm{C}^{18} \mathrm{O}(1-0)$ Galactic Center Survey. The line windows and the $6^{\text {th }}$ order baseline are also displayed. The rms of the fit of the baseline to the spectrum is $0.047 \mathrm{~K}$

for one position was significantly different, first, the line windows were set in a provisional average of the spectra. Then, baselines were subtracted from the individual spectra and these spectra were averaged, weighted by the rms per channel, determined from the baseline fit. Finally, a baseline of order 0 was subtracted from the average spectrum. We have checked that averaging weighted by rms did not have any effect on the intensity and line shape compared to averaging weighted by time. Such an average yielded lower rms per channel only if the noise of the single spectra contributing to one position was significantly different.

The polynomial baseline subtracted was typically of order 6 with long "periods" $\left(\sim 600 \mathrm{~km} \mathrm{~s}^{-1}\right)$ and low amplitudes $(\sim 0.05 \mathrm{~K})$. This baseline was caused by standing waves from reflections in the telescope. The FWZI extent of the $\mathrm{C}^{18} \mathrm{O}$ emission is typically 50 to $250 \mathrm{~km} \mathrm{~s}^{-1}$, which is less than in ${ }^{12} \mathrm{CO}$. In addition, the FWHP width of individual $\mathrm{C}^{18} \mathrm{O}$ emission features are typically about 30 to $60 \mathrm{~km} \mathrm{~s}^{-1}$ which is one order of magnitude less than the period of the subtracted baseline. Therefore, we found that the baselines and $\mathrm{C}^{18} \mathrm{O}$ emission were clearly distinguishable and we could ascertain that the $6^{\text {th }}$ order baselines do not affect the intensities of the $\mathrm{C}^{18} \mathrm{O}$ emission systematically nor introduce artefacts. In Fig. 2, we show a typical example of an averaged spectrum with the chosen emission regions and the subtracted baseline of $6^{\text {th }}$ order.

To check that the observations were reproduceable the innermost positions of the Galactic center region $(l=$ -0.15 to $1.35, b=-0.15$ to 0.0$)$, which were first observed in August 1993, were remeasured in March 1994. In Fig. 3, the integrated intensities, $\int T_{\mathrm{A}}^{*} \mathrm{~d} v$, from both observing periods are compared for the velocity ranges from -373.7 to -225.0 , from -225.0 to +225.0 , and from +225.0 to $+373.7 \mathrm{~km} \mathrm{~s}^{-1}$ which represent the emission of the $\operatorname{HNCO}\left(5_{0,5}-4_{0,4}\right)$ line, the emission of the $\mathrm{C}^{18} \mathrm{O}(1-0)$ line, and an emission-free region, respectively. These plots 
a) Velocity range from -373.729 to $-225.0 \mathrm{~km} \mathrm{~s}^{-1}$
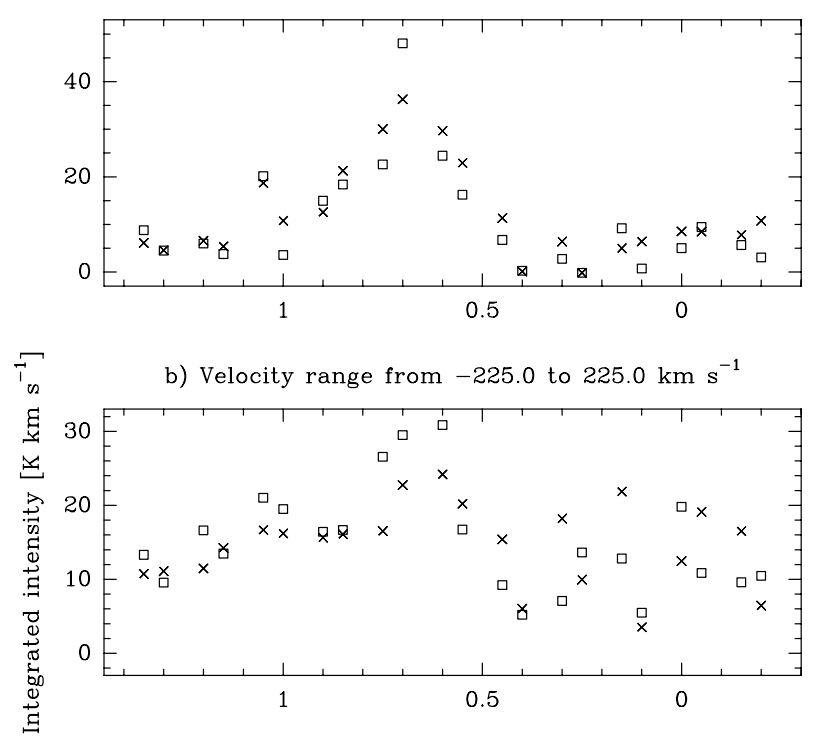

c) Velocity range from 225.0 to $373.729 \mathrm{~km} \mathrm{~s}^{-1}$

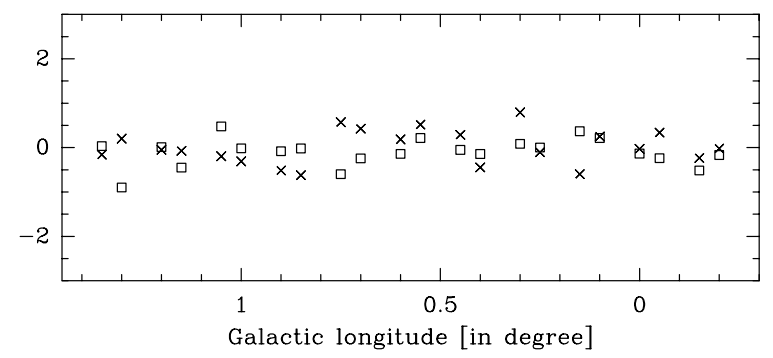

Fig. 3. The integrated intensity of the innermost $b=0^{\circ}$ and $b=-0.15$-positions of the Galactic center region which were measured twice. The open squares represent the results of August 1993, the crosses the results of March 1994. The positions at $b=-0.15$ are plotted with their $l$ value reduced by 0.05

show that the observations were reproducible within a certain scatter and that no time-dependent systematic changes were present in the data. A good measure for the scatter is the mean deviation of the integrated intensity of the two periods which is given by:

$$
\Delta I_{i}=\sqrt{\left(I_{i}^{1993}\right)^{2}+\left(I_{i}^{1994}\right)^{2}-\frac{1}{2}\left(I_{i}^{1993}+I_{i}^{1994}\right)^{2}}
$$

where $I_{i}$ is the integrated intensity of position $i$. The average value of this mean deviation, $\Delta I$, is given in Table 1 and compared to the expected value, $\Delta I_{\mathrm{ev}}$, from the average rms per channel, $0.04 \mathrm{~K}$, determined by:

$\Delta I_{\mathrm{ev}}=\sqrt{N_{\mathrm{ch}}} v_{\mathrm{ch}} \mathrm{rms}$

where $N_{\text {ch }}$ is the number of channels covered by the integration area and $v_{\mathrm{ch}}$ is the channel width. From this comparison, it becomes clear that in the emission-free area the scatter is within the expected limit whereas in the areas containing emission it is larger. This indicates that the uncertainties of the integrated intensities in areas of emission is mostly caused by uncertainties in the baselines and that the rms noise per channel plays a minor role. The reason for the rather strong influence of the baseline choice on the integrated intensity is that the observed emission lines are rather weak but have extended wings. Therefore, a small error in the baseline causes a relatively large error in the integrated intensity. This effect is even larger for the $\operatorname{HNCO}\left(5_{0,5}-4_{0,4}\right)$ line due to additional uncertainties caused by this line being at the edge of the frontend and backend bands. It illuminates the necessity of having not only a sensitive system but also a stable system with flat baselines when broad lines are observed (see also Radford et al. 1996 for an analysis of baseline problems when observing broad, in their case extragalactic, lines).

Table 1. The averaged relative deviation of the integrated intensity of the innermost Galactic center region of the two observation shifts in August 1993 and March 1994 and the expected deviation

\begin{tabular}{cccc}
$\begin{array}{c}\text { Velocity range } \\
\mathrm{km} \mathrm{s}^{-1}\end{array}$ & Line & $\begin{array}{c}\Delta \boldsymbol{I} \\
\mathrm{K} \mathrm{km} \mathrm{s}^{-1}\end{array}$ & $\begin{array}{c}\Delta \boldsymbol{I}_{\mathbf{e v}} \\
\mathrm{K} \mathrm{km} \mathrm{s}^{-1}\end{array}$ \\
\hline$-373.7--225.0$ & $\mathrm{HNCO}$ & 7.10 & 0.84 \\
$-225.0-+225.0$ & $\mathrm{C}^{18} \mathrm{O}$ & 5.42 & 1.45 \\
$+225.0-+373.7$ & - & 0.25 & 0.84 \\
\hline
\end{tabular}

\subsection{Data calibration}

The calibration of the scans follows the standard chopper wheel method first described by Penzias \& Burrus (1973) and applied at many other millimeter telescopes. See Appendix A.2 for a detailed description of this method.

\subsubsection{Calibration stability}

In addition to daily antenna tippings, the calibration stability was monitored by taking a 10-minute-spectrum toward the position $l=0.625, b=0.0$ (Sgr B2) once or twice per day. With a peak temperature of $1.14 \mathrm{~K}$, the $\operatorname{HNCO}\left(5_{0,5}-4_{0,4}\right)$ line is considerably stronger than the $\mathrm{C}^{18} \mathrm{O}(1-0)$ line $(0.45 \mathrm{~K})$ toward this position and its intensity, integrated over a velocity range from -322.0 to $-245.0 \mathrm{~km} \mathrm{~s}^{-1}$ (with respect to the $\mathrm{C}^{18} \mathrm{O}(1-0)$ line frequency) is a good indicator for the calibration stability.

The stability of the calibration, throughout the course of the survey, proved to be excellent (better than $1 \%$ on different days), and the mean uncertainty in the calibration of a single scan is $\sim 20 \%$. For 12 scans, the typical number of scans per position, the calibration uncertainty just from statistics is $\sim 6 \%$ and probably better, because a large part of the calibration uncertainty is caused by 
the uncertainty in the determination of the baseline which was much better determined in the averaged spectrum (see also Sect. 3.3).

\subsubsection{Calibration scaling of the AOS spectra}

The chopper wheel method calibrates the signal coming out of the backend to the antenna temperature, $T_{\mathrm{A}}^{*}$. This calibration bases on the scaling factor $T_{\mathrm{CAL}}$ (see Appendix A.2), which, for instrumental reasons, could be determined only with the filterbank. Because the signal path to the AOS differed from that to the filterbank, the response of the two backends was not equal. Therefore, as a last step in the calibration process, the correct scaling of the spectra taken with the AOS to $T_{\mathrm{A}}^{*}$, the AOS "efficiency", had to be determined. The effect of different response could be seen most clearly toward positions where the $\mathrm{C}^{18} \mathrm{O}(1-0)$ line emission was narrow enough and close enough to $v_{\mathrm{LSR}}=0 \mathrm{~km} \mathrm{~s}^{-1}$ to be also observed with the filterbank. A comparison of more than 150 spectra, taken simultaneously with the AOS and the filterbank toward 13 different positions, yielded a ratio of peak antenna temperatures between the AOS and the filterbank, resampled to the resolution of the AOS, of $1.34 \pm 0.18$, and a ratio of $1.46 \pm 0.33$ for integrated intensities (see Dahmen 1995 for more details).

However, to finally determine the different scaling factor of the AOS it is necessary to compare an emission feature of the AOS, which is well resolved and which lies well above the detection limit, with the result of an observation toward the same position with the same beamwidth and with comparable frequency resolution the calibration of which is well known. Obviously, $\operatorname{Sgr} \mathrm{B} 2(l=0.625$, $b=0.0)$ is a suitable position for this purpose. Sgr B2 was observed in $\mathrm{C}^{18} \mathrm{O}(1-0)$ with the $0.5 \mathrm{MHz}$-resolutionfilterbank at the $1.2 \mathrm{~m}$ NMWT during November 1994. This telescope has the same beamwidth as the SMWT and the calibration is well established (Cohen et al. 1986). Because the scaling factor from $T_{\mathrm{A}}^{*}$ to $T_{\mathrm{MB}}, \eta_{\mathrm{mf}}=\frac{B_{\text {eff }}}{F_{\text {eff }}}$, is the same for both telescopes (see Appendix A.2) the $T_{\mathrm{A}}^{*}$ values could be compared directly. This comparison was done using peak intensities instead of integrated intensities because a significant part of the integrated intensity originates in the extended wings of the broad line. Thus, small errors in the baseline give rise to larger errors in the integrated intensity, since the integrated intensity is the product of the velocity window and the average intensity in this window. An identical absolute baseline error is translated to a larger relative error if related to the average intensity than if related to the peak intensity. In both spectra, the peak intensity was fitted, with a Gaussian curve. With these fits, the "efficiency" of the AOS calibration at the $\mathrm{C}^{18} \mathrm{O}(1-0)$ line emission, $\eta_{\mathrm{AOS}-\mathrm{C}^{18} \mathrm{O}}$, is:

$\eta_{\mathrm{AOS}-\mathrm{C}^{18} \mathrm{O}}=1.320 \pm 0.172$.
This value is, within the noise, the same as the one determined by comparing spectra obtained with the AOS with spectra obtained with the FB at the $1.2 \mathrm{~m}$ SMWT. Thus, the main (and probably single) cause for scaling differences between spectra taken with the SMWT and the $1.2 \mathrm{~m} \mathrm{NMWT}$ is the integration of the AOS into the system of the SMWT. The calibration of the SMWT filterbank is identical to within the noise with the calibration obtained at the NMWT, and the following final conversions for $T_{\mathrm{A}}^{*}$ and $T_{\mathrm{MB}}$ can be established:

$$
\begin{aligned}
T_{\mathrm{A}}^{*} & =\frac{T_{\mathrm{A}-\mathrm{AOS}}^{*}}{\eta_{\mathrm{AOS}-\mathrm{C}^{18} \mathrm{O}}}=\frac{T_{\mathrm{A}-\mathrm{AOS}}^{*}}{1.320} \\
T_{\mathrm{MB}} & =\frac{T_{\mathrm{A}}^{*}}{\eta_{\mathrm{mf}}}=\frac{T_{\mathrm{A}-\mathrm{AOS}}^{*}}{\eta_{\mathrm{mf}} \eta_{\mathrm{AOS}-\mathrm{C}^{18} \mathrm{O}}}=\frac{T_{\mathrm{A}-\mathrm{AOS}}^{*}}{1.082}
\end{aligned}
$$

\section{Results of the $\mathrm{C}^{18} \mathrm{O}(\mathbf{1}-0)$ Galactic Center Survey}

In this section, we present the results of the $\mathrm{C}^{18} \mathrm{O}(1-0)$ Galactic Center Survey. In Fig. 4, the full set of survey spectra is given. The mean rms noise per channel of all 357 spectra is $36.0 \pm 6.6 \mathrm{mK}$. Note that the strongest emission, which appears in the inner Galactic center region at velocities more negative than $-200 \mathrm{~km} \mathrm{~s}^{-1}$, is attributed to the $\operatorname{HNCO}\left(5_{0,5}-4_{0,4}\right)$ line.

\subsection{The integrated intensity}

\subsubsection{The total integrated $\mathrm{C}^{18} \mathrm{O}$ emission}

In Fig. 5, we show a contour map of the integrated intensity, $\int T_{\mathrm{MB}} \mathrm{d} v$, of the $\mathrm{C}^{18} \mathrm{O}(1-0)$ line, covering the velocity range from -225.0 to $+225.0 \mathrm{~km} \mathrm{~s}^{-1}$, i.e., the complete emission range of the Galactic center region. To produce smooth contours in the contour map the number of points in the data base for the contour map was increased by a factor of 5 per axis with a standard CLASS interpolation routine, resulting in an increase in the density of points by a factor of 25 . The main $\mathrm{C}^{18} \mathrm{O}$ emission regions coincide with the known continuum and CO peaks Sgr A (with the extension to Sgr B1), Sgr B2 (Lequeux 1962), Sgr C and Sgr D (Hoffmann et al. 1971). In addition, Clump 2 (Bania 1977) in the range from $l=3.0$ to 3.5 is weak but visible. The most prominent source by far, both in Fig. 5 and, even more strikingly, in the channel maps (Fig. 6) is Sgr B2. The sources G 0.50 .0 and G $0.9+0.1$ (Downes \& Maxwell 1966), which are distinct emission peaks in radio continuum maps (see e.g. Reich et al. 1984 at $2.695 \mathrm{GHz}$, Altenhoff et al. 1978 at $4.875 \mathrm{GHz}$, and Handa et al. 1987 at 10.05 and $10.55 \mathrm{GHz}$ ), are not visible. In Table 2 , the coordinates of the main radio and FIR continuum emission sources are summarized. 


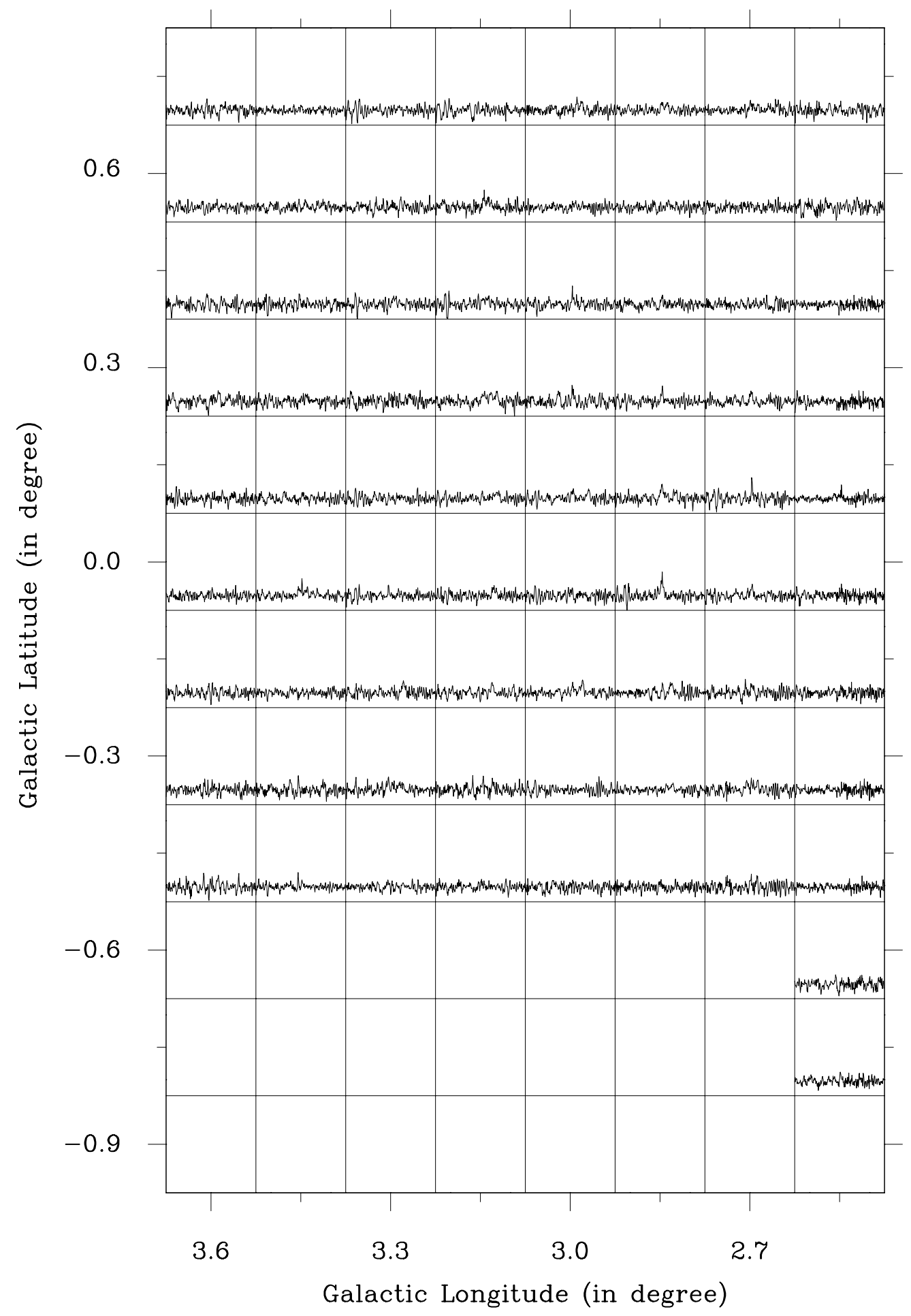

Fig. 4. The full set of spectra of the $\mathrm{C}^{18} \mathrm{O}(1-0)$ Galactic Center Survey. The $x$-axis of the spectra is $v_{\text {LSR }}$ with respect to the $\mathrm{C}^{18} \mathrm{O}(1-0)$ line frequency and ranges from -375.2 to $+375.2 \mathrm{~km} \mathrm{~s}^{-1}$, the $y$-axis is $T_{\mathrm{MB}}$ and ranges from -0.15 to $0.85 \mathrm{~K}$. The latitude range is, in every case, from $b=-0.9$ to +0.75 . Note that the strongest emission, which appears in the inner Galactic center region at velocities lower than $-200 \mathrm{~km} \mathrm{~s}^{-1}$, is caused by the $\operatorname{HNCO}\left(5_{0,5}-4_{0,4}\right)$ line. a) Longitude range from $l=3.6$ to 2.55 


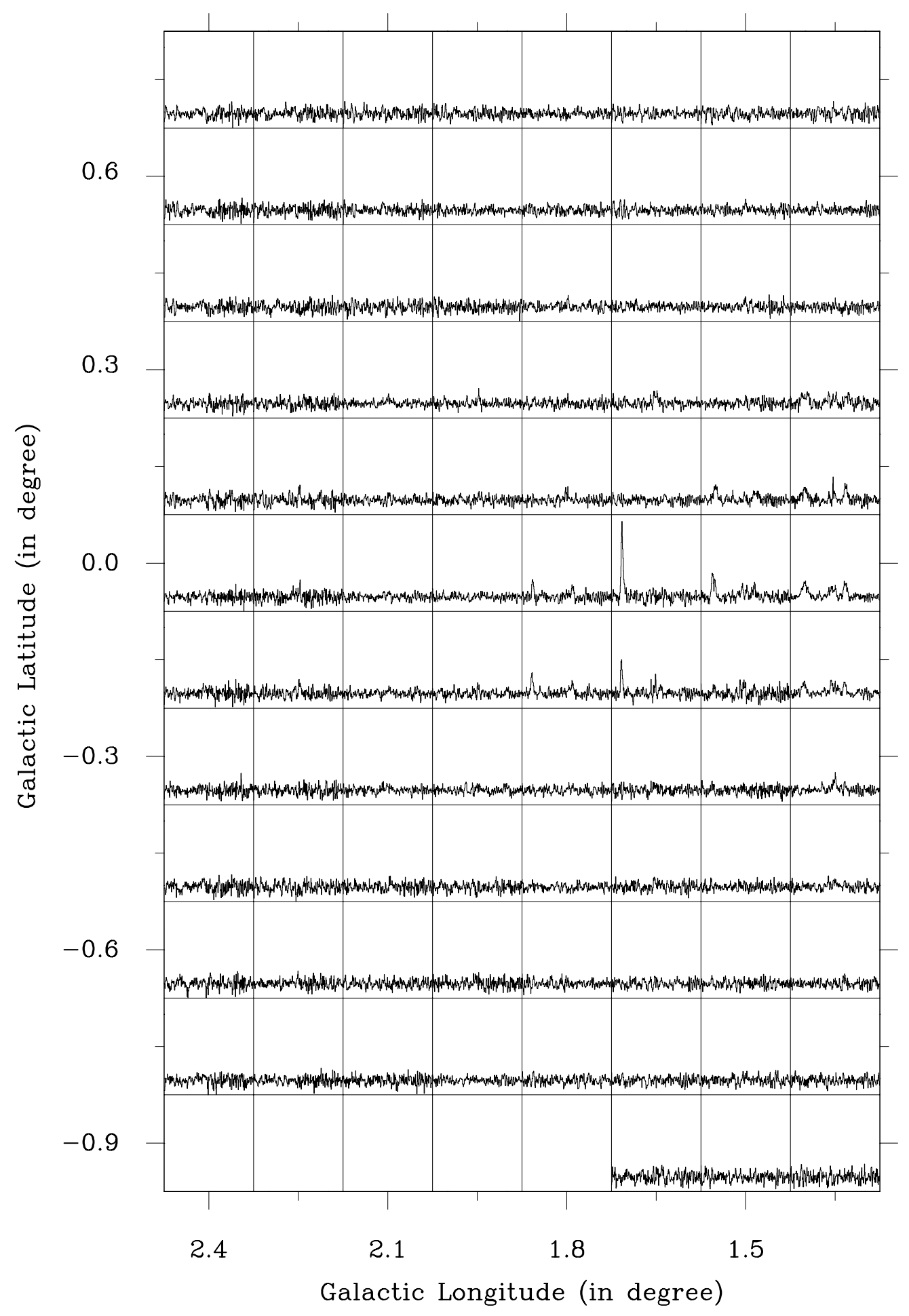

Fig. 4. b) Longitude range from $l=2.4$ to 1.35

4.1.2. Channel maps of $50 \mathrm{~km} \mathrm{~s}^{-1}$ width

In Fig. 6, we present channel maps integrated over $50 \mathrm{~km} \mathrm{~s}^{-1}$ windows. The central velocity of these maps ranges from $-200 \mathrm{~km} \mathrm{~s}^{-1}$ to $+200 \mathrm{~km} \mathrm{~s}^{-1}$.
At $-200 \mathrm{~km} \mathrm{~s}^{-1}$, no $\mathrm{C}^{18} \mathrm{O}$ is visible above the noise; the feature at $(l=1.0, b=-0.15)$ is caused by the $\operatorname{HNCO}\left(5_{0,5}-4_{0,4}\right)$ line the emission of which extends to 


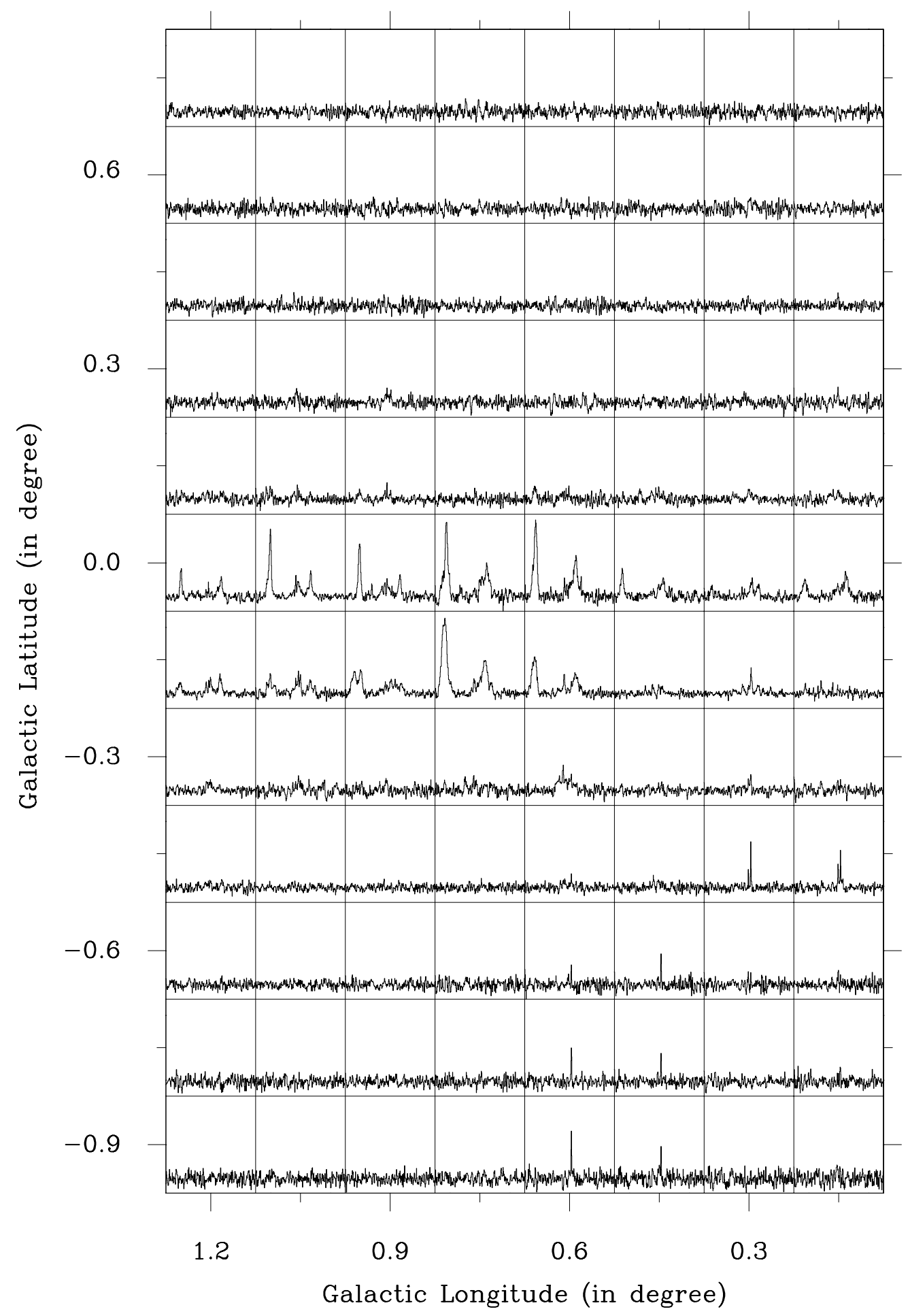

Fig. 4. c) Longitude range from $l=1.2$ to 0.15

more than $+100 \mathrm{~km} \mathrm{~s}^{-1}$ at this position (compare the respective feature of $\mathrm{C}^{18} \mathrm{O}$ in the top panel of Fig. 6c).
At $-150 \mathrm{~km} \mathrm{~s}^{-1}$ and at $-100 \mathrm{~km} \mathrm{~s}^{-1}$, only marginal $\mathrm{C}^{18} \mathrm{O}$ emission is visible, mainly at $\operatorname{Sgr} \mathrm{C}(l=-0.5, b=$ $0.0)$ and between $l=0^{\circ}$ and $1^{\circ}$ at about $b=-0^{\circ} 3$. 


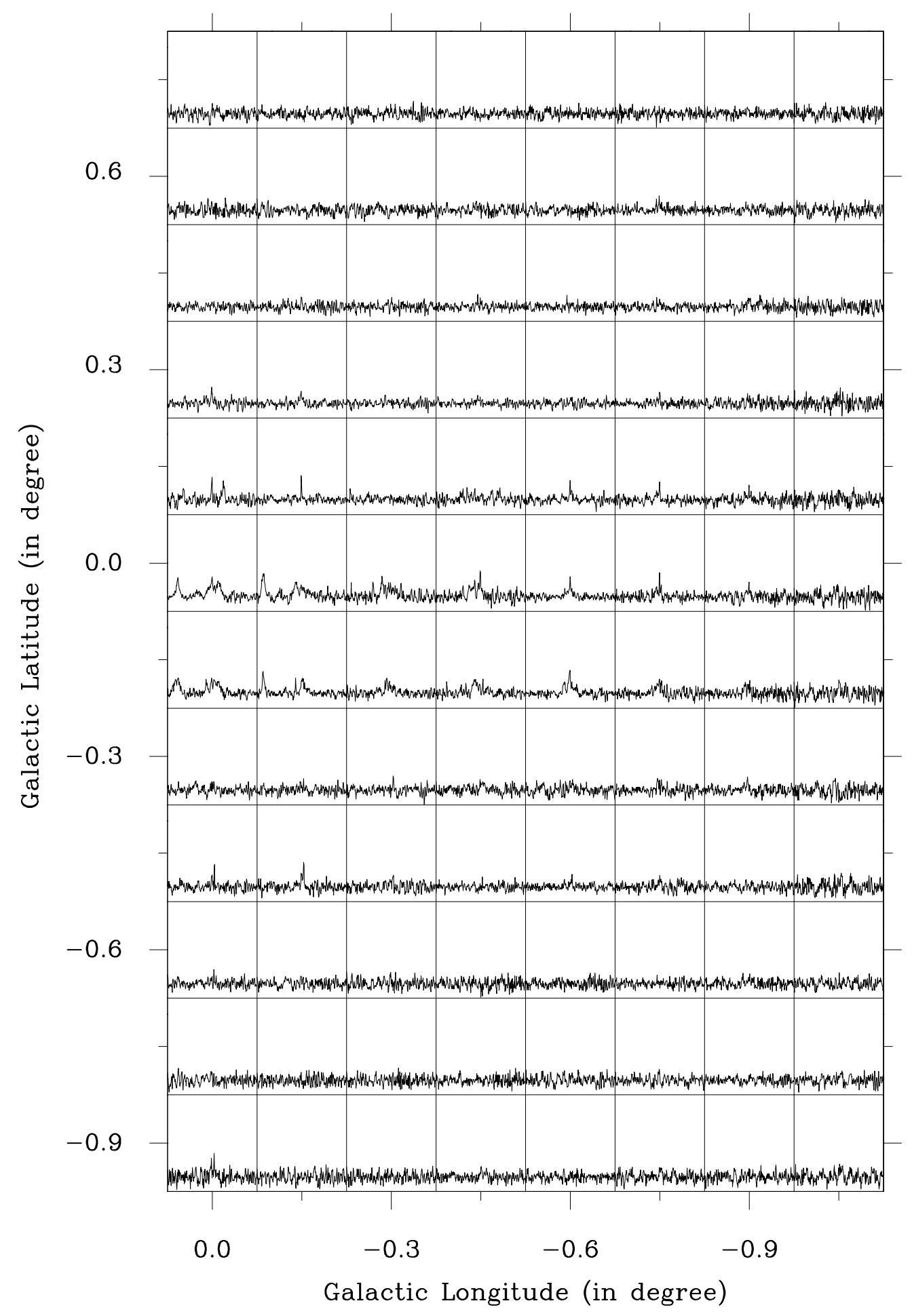

Fig. 4. d) Longitude range from $l=0.0$ to -1.05

At $-50 \mathrm{~km} \mathrm{~s}^{-1}$, the rather widespread emission of the 3 -kpc-arm (Rougoor \& Oort 1960) can be seen; this peaks at $(l=-0.4, b=-0.1)$ and at $(l=0.6, b=-0.3)$. The weak emission maximum in the area of $l=0.8$ to
1.1 and $b=-0.2$ to +0.15 might correspond to the radio continuum source G $0.9+0.1$.

At $0 \mathrm{kms}^{-1}$, the Galactic center emission is superposed on the emission of local gas. The local component 


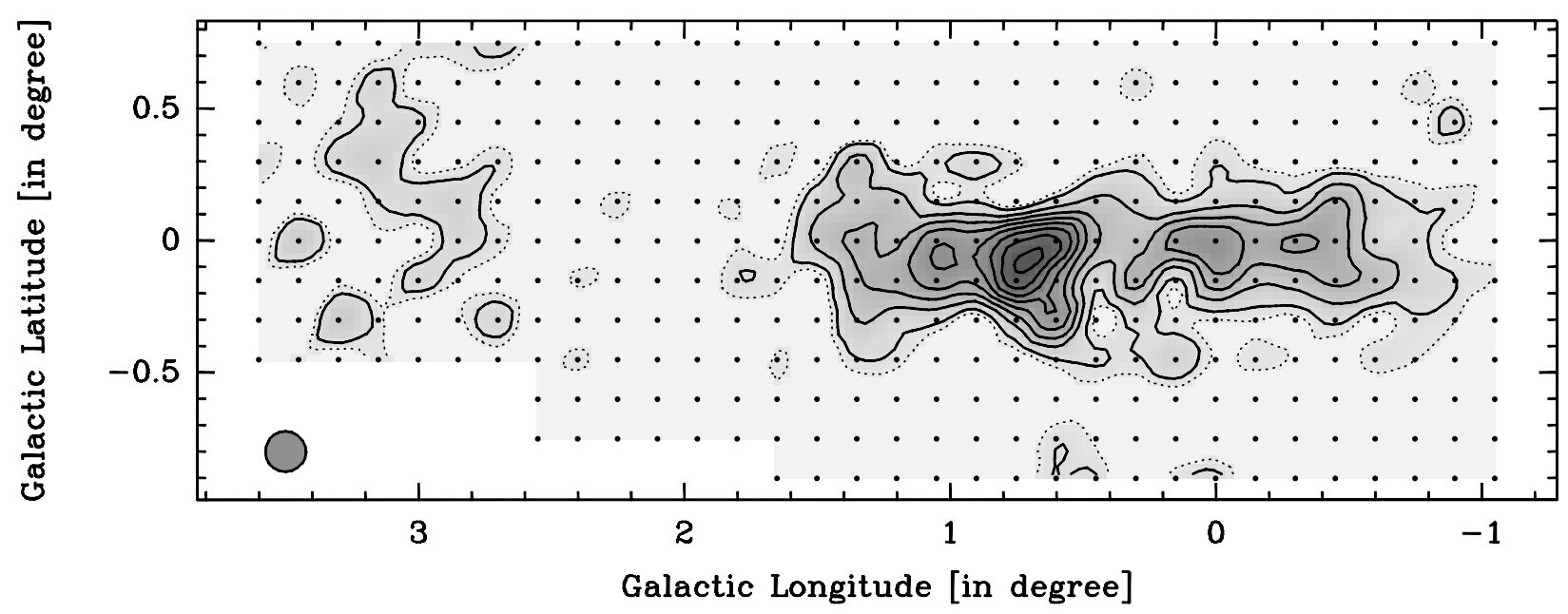

Fig. 5. The integrated intensity of the Galactic center region in $\mathrm{C}^{18} \mathrm{O}(1-0)$. The velocity over which the intensity is integrated ranges from -225.0 to $+225.0 \mathrm{~km} \mathrm{~s}^{-1}$. The solid contour levels range from 3.9 to 28.05 in steps of $3.45 \mathrm{~K} \mathrm{~km} \mathrm{~s}^{-1}$ where the lowest level is the $3 \sigma$-value. The dashed contour is at $2.6 \mathrm{~K} \mathrm{~km} \mathrm{~s}^{-1}$ which is the $2 \sigma$-value. The circle in the lower left corner of the plot indicates the beam size of 9 '.

Table 2. The main radio and FIR continuum emission sources of the inner Galactic center region

\begin{tabular}{|c|c|c|c|c|c|}
\hline $\begin{array}{l}\text { Source } \\
\text { name }\end{array}$ & $\begin{array}{l}\text { Radio } \\
\text { name }\end{array}$ & $\begin{array}{l}\text { Equatorial } \\
\text { R.A.(1950) }\end{array}$ & $\begin{array}{l}\text { Coordinates } \\
\text { Decl.(1950) }\end{array}$ & $\begin{array}{c}\text { Galacti } \\
l^{\text {II }}\end{array}$ & $\begin{array}{c}\text { Coordinates } \\
b^{\text {II }}\end{array}$ \\
\hline Sgr A & G $0.00 .0^{\mathrm{b}}$ & $17^{\mathrm{h}} 42^{\mathrm{m}} 30^{\mathrm{s}} \mathrm{f}$ & $-28^{\circ} 59^{\prime} 06^{\prime \prime f}$ & -0.052 & -0.046 \\
\hline $\mathrm{Sgr} B 1^{\mathrm{a}}$ & G $0.20 .0^{\mathrm{b}}$ & $17^{\mathrm{h}} 43^{\mathrm{m}} 01^{\mathrm{s}} \mathrm{f}$ & $-28^{\circ} 47^{\prime} 12^{\prime \prime} \mathrm{f}$ & 0.177 & -0.038 \\
\hline $\mathrm{Sgr} \mathrm{B} 2^{\mathrm{a}}$ & G $0.70 .0^{\mathrm{b}}$ & $17^{\mathrm{h}} 44^{\mathrm{m}} 09^{\mathrm{s}} \mathrm{f}$ & $-28^{\circ} 21^{\prime} 54^{\prime \prime \mathrm{f}}$ & 0.666 & -0.030 \\
\hline $\operatorname{Sgr} C^{c}$ & $\mathrm{G}-0.6-0.1^{\mathrm{b}}$ & $17^{\mathrm{h}} 41^{\mathrm{m}} 26^{\mathrm{s}} \mathrm{f}$ & $-29^{\circ} 27^{\prime} 18^{\prime \prime \mathrm{f}}$ & -0.574 & -0.096 \\
\hline Sgr $D^{c}$ & $\mathrm{G} 1.1-0.1^{\mathrm{b}}$ & $17^{\mathrm{h}} 45^{\mathrm{m}} 33^{\mathrm{s}} \mathrm{f}$ & $-28^{\circ} 00^{\prime} 30^{\prime \prime \mathrm{f}}$ & 1.131 & -0.107 \\
\hline FIR $18^{\mathrm{f}}$ & G $0.50 .0^{\mathrm{b}}$ & $17^{\mathrm{h}} 43^{\mathrm{m}} 53^{\mathrm{s}} \mathrm{f}$ & $-28^{\circ} 30^{\prime} 12^{\prime \prime} \mathrm{f}$ & 0.518 & -0.052 \\
\hline No. $71^{\mathrm{d}}$ & G $0.9+0.1^{\mathrm{b}}$ & $17^{\mathrm{h}} 44^{\mathrm{m}} 12^{\mathrm{s}} \mathrm{e}$ & $-28^{\circ} 08^{\prime} 24^{\prime \prime}{ }^{\mathrm{e}}$ & 0.863 & +0.080 \\
\hline \multirow{3}{*}{\multicolumn{3}{|c|}{${ }^{a}$ from Lequeux (1962) }} & \multicolumn{3}{|c|}{${ }^{\mathrm{d}}$ from Downes et al. (1978) } \\
\hline & & & \multicolumn{3}{|c|}{ e from Altenhoff et al. (1978) } \\
\hline & & & \multicolumn{3}{|c|}{${ }^{\mathrm{f}}$ from Odenwald \& Fazio (1984). } \\
\hline
\end{tabular}

can be distinguished by inspecting the individual spectra; the linewidth of this emission is comparatively narrow $\left(\sim 5 \mathrm{~km} \mathrm{~s}^{-1} \mathrm{FWHM}\right)$ whereas Galactic center emission is characterized by its broad $\left(\sim 50 \mathrm{~km} \mathrm{~s}^{-1} \mathrm{FWHM}\right)$ lines. From this, it is clear that the emission in the area of $l=-0.3$ to 0.6 and $b=-0.9$ to -0.3 is almost exclusively of local origin whereas the emission elsewhere is dominated by Galactic center gas. The Galactic center gas is most widespread and uniformly distributed at this velocity. The Galactic center emission peaks at Sgr A $(l=0.0, b=0.0)$, at $(l=0.75, b=-0.1)$, somewhat east of Sgr B2, and at $(l=-0 \circ 6, b=-0.15)$ (roughly Sgr C). The emission covers the inner region of the Galactic cen- ter completely, as seen in the contour map of the total emission of the Galactic center region (Fig. 5). In addition, the emission extends to $b$ values up to +0.6 and fills the gap between the inner region and Clump 2. It is possible that the remaining narrow gap at $l=2{ }^{\circ} 4$ is connected to Clump 2 by the weak emission at $-50 \mathrm{~km} \mathrm{~s}^{-1}$ at this longitude. Clump 2 has weak but clearly visible emission in the area of $l=2.6$ to $3.0, b=-0.15$ to +0.35 and around $(l=2.6, b=0.0)$.

At $+50 \mathrm{~km} \mathrm{~s}^{-1}, \mathrm{Sgr}$ B2 is the most prominent emission feature by far. It peaks at $(l=0.68, b=-0.06)$, and it is, with an integrated intensity of more than $15 \mathrm{~K} \mathrm{~km} \mathrm{~s}^{-1}$, the strongest emission feature in all the channel maps. 

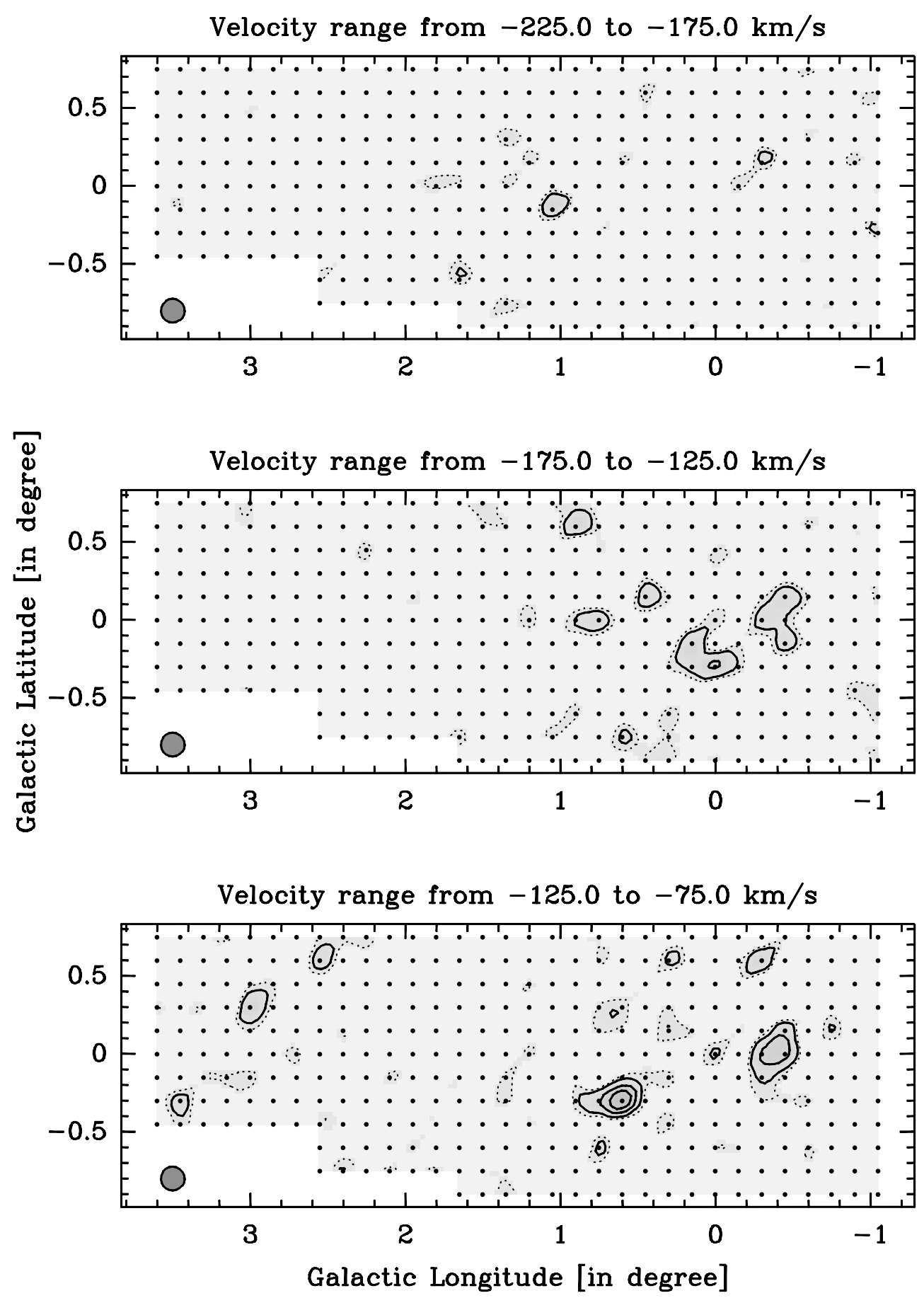

Fig. 6. The integrated intensity of the Galactic center region in $\mathrm{C}^{18} \mathrm{O}(1-0)$ in velocity intervals of $50 \mathrm{~km} \mathrm{~s}^{-1}$ width. The solid contour levels start at $1.3 \mathrm{~K} \mathrm{~km} \mathrm{~s}^{-1}$, which is the $3 \sigma$-level, and increase in steps of $1.15 \mathrm{~K} \mathrm{~km} \mathrm{~s}^{-1}$. The dashed contour is at $0.8667 \mathrm{~K} \mathrm{~km} \mathrm{~s}^{-1}$ which is the $2 \sigma$-value. The circle in the lower left corner of the plots indicates the beam size of 9'2. a) At the top the integrated intensity in the velocity range from -225.0 to $-175.0 \mathrm{~km} \mathrm{~s}^{-1}$ is plotted, in the middle panel the velocity ranges from -175.0 to $-125.0 \mathrm{~km} \mathrm{~s}^{-1}$, and at the bottom the velocity range from -125 to $-75.0 \mathrm{~km} \mathrm{~s}^{-1}$ is shown 

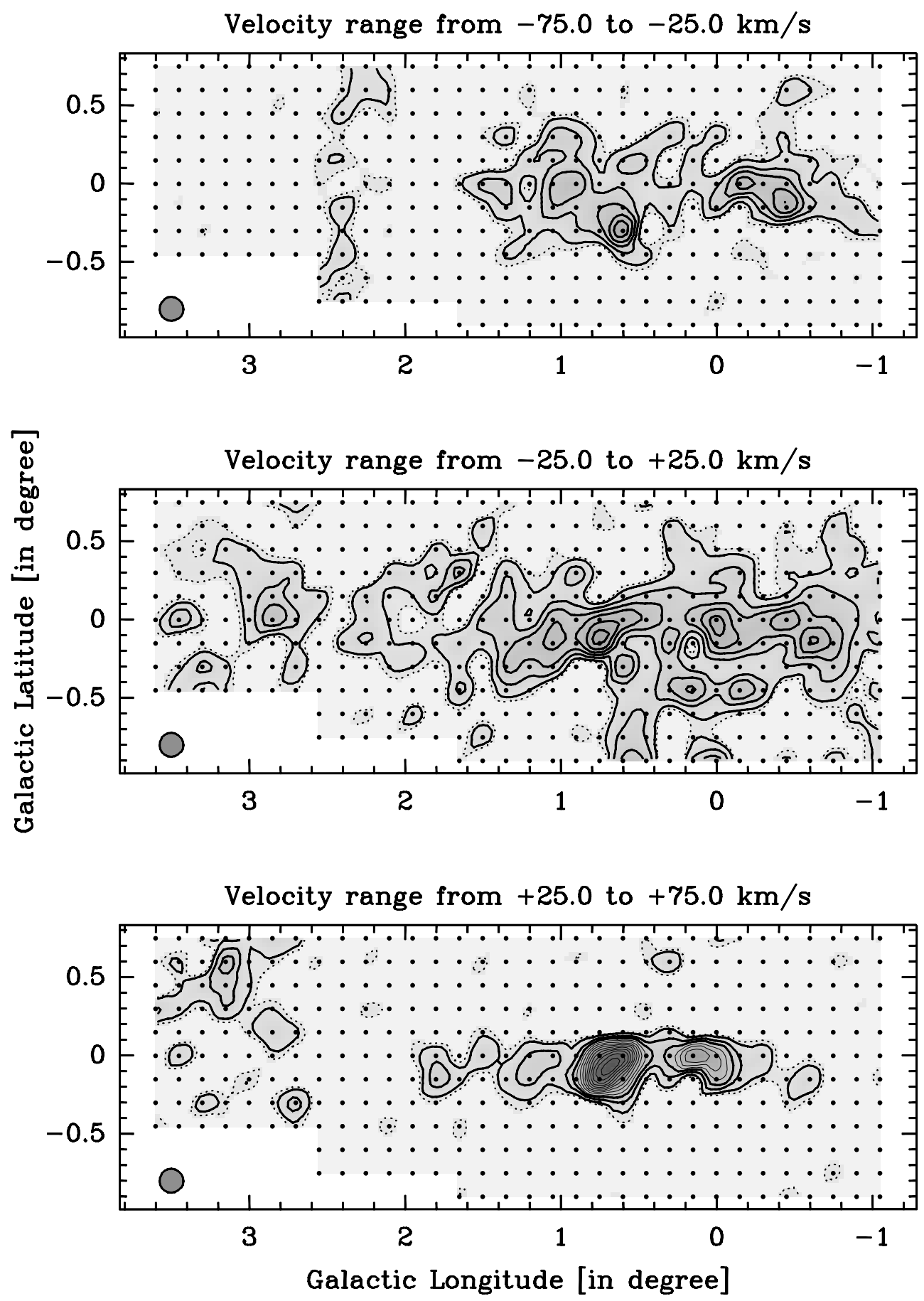

Fig. 6. b) At the top the integrated intensity of the velocity range from -75.0 to $-25 \mathrm{~km} \mathrm{~s}^{-1}$ is plotted, in the middle the velocity ranges from -25 to $+25 \mathrm{~km} \mathrm{~s}^{-1}$, and at the bottom the velocity range from +25 to $+75.0 \mathrm{~km} \mathrm{~s}^{-1}$ is shown

Compared to Sgr B2, the emission from Sgr A is rather weak, and $\mathrm{Sgr} \mathrm{C}$ and D are only marginally visible. Again, Clump 2 appears weak but is clearly visible. However, at this velocity the emission peaks at $(l=3.15, b=+0.6)$ and covers the area of $l=3.0$ to $3.3, b=+0.3$ to +0.7 with extensions to $(l=2.8, b=+0 \circ 1)$ and $(l=3.6$, 

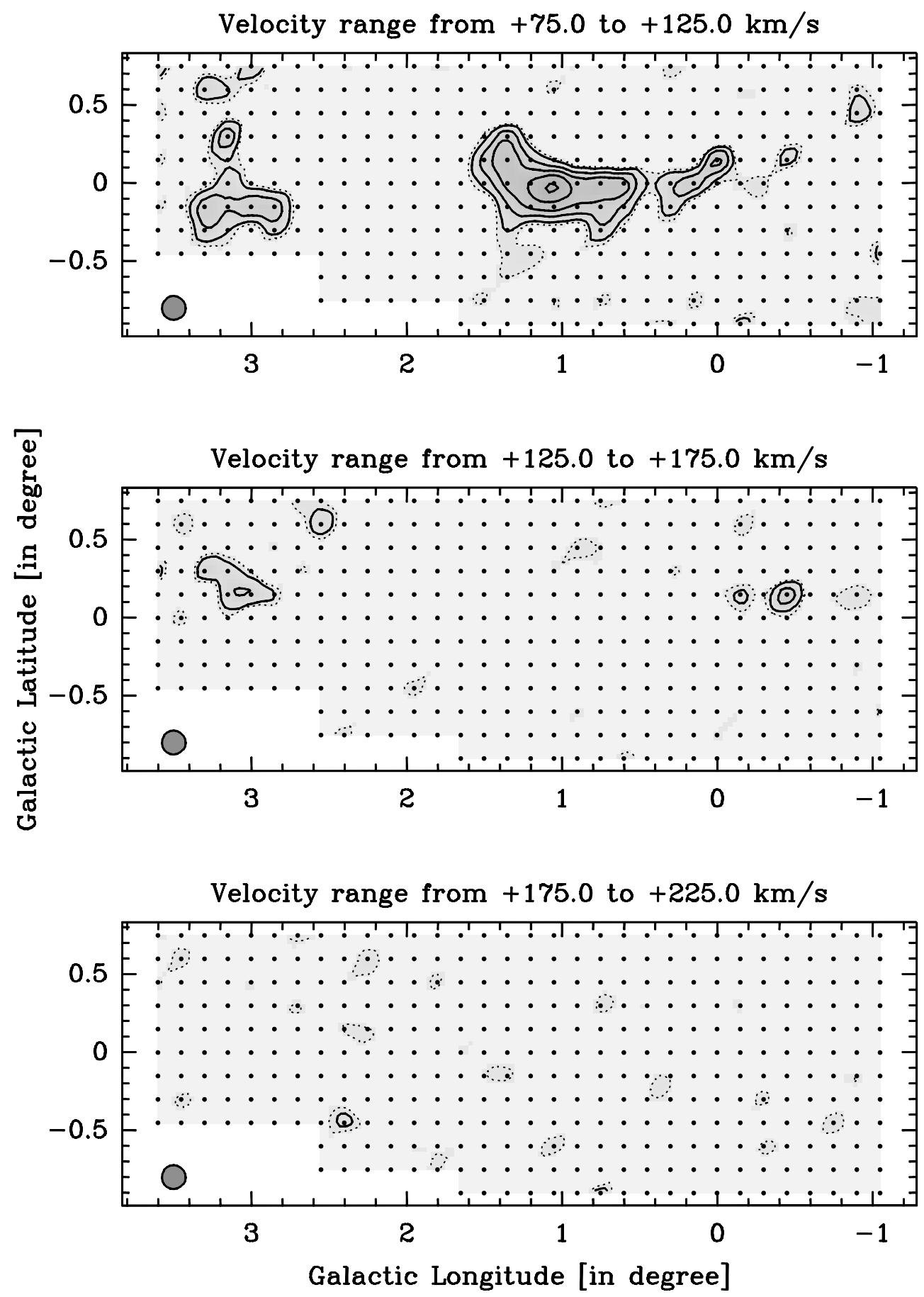

Fig. 6. c) At the top the integrated intensity of the velocity range from +75.0 to $+125 \mathrm{~km} \mathrm{~s}^{-1}$ is plotted, in the middle the velocity ranges from +125 to $+175 \mathrm{~km} \mathrm{~s}^{-1}$, and at the bottom the velocity range from +175 to $+225.0 \mathrm{~km} \mathrm{~s}^{-1}$ is shown

$b=+0$. 3$)$. In addition, the emission of Clump 2 might be extended to $b$ values beyond the map at $(l=3$. $1, b=$ +0 .75) and to larger $l$ values at $(l=3.6, b=+0.3)$.
At $+100 \mathrm{~km} \mathrm{~s}^{-1}$, Sgr D is the dominant emission feature (called the $l=1.5$-complex in Bally et al. 1988). It peaks at $(l=1.0, b=0.0)$ and ranges from 0.5 to 1.5 
in $l$ and from -0.3 to $+0 \circ 1$ in $b$. At longitudes greater than 1.0, this emission feature curves upward to a latitude of +0.4 at $l=1.35$. Apart from this main feature, some emission is visible near Sgr A. Clump 2 peaks in the area of $l=2.8$ to $3 \circ 4, b=-0.3$ to 0.0 with an extension to $(l=3.15, b=+0 \circ 3)$. Some additional emission in the area of $l=3.0$ to $3.4, b=+0.55$ to +0.75 seems to be present.

At $+150 \mathrm{~km} \mathrm{~s}^{-1}$, nearly no $\mathrm{C}^{18} \mathrm{O}$ emission was observed at our detection level. Some marginal features appear between Sgr A and Sgr C. However, Clump 2 is still visible in the area of $l=2.85$ to $3.35, b=+0.05$ to +0.4 .

At $+200 \mathrm{~km} \mathrm{~s}^{-1}$, no $\mathrm{C}^{18} \mathrm{O}$ emission is visible.

The channels at very high velocity $\left(+250 \mathrm{~km} \mathrm{~s}^{-1}\right.$ to $+350 \mathrm{~km} \mathrm{~s}^{-1}$ ) were also checked for emission. As expected from the ${ }^{12} \mathrm{CO}$ data, with one exception they show no emission in $\mathrm{C}^{18} \mathrm{O}$ : At $+250 \mathrm{~km} \mathrm{~s}^{-1}$ some marginal emission might be visible in the area of $l=1.9$ to $2.4, b=-0.3$ to -0.1 .

\subsection{Longitude-velocity plots}

\subsubsection{Plots integrated over ranges of latitude}

In Fig. 7, the intensity integrated over two latitude ranges is plotted. These longitude-velocity plots ( $l v$-plots) show the velocity distribution of the $\mathrm{C}^{18} \mathrm{O}(1-0)$ emission a) in the complete latitude range from -0.90 to +0.75 and $\mathrm{b}$ ) in the inner latitude range from -0.30 to +0.30 . To improve the signal-to-noise ratio, four velocity channels were averaged so that the velocity resolution is about $11.7 \mathrm{~km} \mathrm{~s}^{-1}$. The dots in the plot indicate the remaining data points in $l$ and $v$. The data for the contour maps were interpolated between these points, increasing the number of points by a factor of 5 per axis. The $1 \sigma$-value was calculated using the following equation:

$\sigma_{l v}=\sqrt{N_{b-\mathrm{pts}}} b_{b-\mathrm{pts}} \mathrm{rms}$

where $N_{b-\text { pts }}$ is the number of latitude points covered by the latitude integration - which was 12 in case a) and 5 in case b) - and $b_{b-\text { pts }}$ is the spacing in latitude which was 0.15 in both cases. The mean rms per channel used was $16.85 \mathrm{mK}$, the value reached by averaging four velocity channels.

In general, both plots show the same features. In plot a) one finds that Clump 2, which is more extended in $b$, is more widespread in the $l v$-space whereas in plot b) the features toward Sgr A to D, which are more concentrated toward the plane, stand out more clearly. The emission of local gas and of Galactic center gas almost at rest with respect to the LSR frame appears mainly in a line from $l=-1.05$ to +1.8 at about $-5 \mathrm{~km} \mathrm{~s}^{-1}$. At +1.8 , the emission shifts to a center of about $+10 \mathrm{~km} \mathrm{~s}^{-1}$ and continues to +3.3 at about $+20 \mathrm{~km} \mathrm{~s}^{-1}$. The 3 -kpc-arm at about $-50 \mathrm{~km} \mathrm{~s}^{-1}$ is visible in the range from -0.6 to $+1.5 \mathrm{but}$ the emission is not very homogeneous. In the range from
-0.6 to 0.0 ( $\operatorname{Sgr} \mathrm{C}$ ) and from +0.6 to +1.5 (Sgr B2 and $\mathrm{D})$, there are large transition areas between the 3 -kpc-arm gas and the gas at rest with respect to the LSR frame. The most prominent feature is the diagonal chain of emission centers ranging from $\operatorname{Sgr} \mathrm{C}\left(l \approx-0.6, v \approx-50 \mathrm{~km} \mathrm{~s}^{-1}\right)$ over $\operatorname{Sgr} \mathrm{A}\left(l \approx 0.0, v \approx-5 \mathrm{~km} \mathrm{~s}^{-1}\right), \operatorname{Sgr} \mathrm{B} 1(l \approx+0.3$, $\left.v \approx+15 \mathrm{kms}^{-1}\right), \operatorname{SgrB} 2\left(l \approx+0.65, v \approx+50 \mathrm{kms}^{-1}\right)$ to $\operatorname{Sgr} \mathrm{D}\left(l \approx+1.3, v \approx+90 \mathrm{~km} \mathrm{~s}^{-1}\right)$. Note the weak tail of emission which ranges from $\operatorname{Sgr} \mathrm{A}$ to about $(l \approx+0.3$, $\left.v \approx+80 \mathrm{~km} \mathrm{~s}^{-1}\right)$. At $\left(l \approx+1.8, v \approx+50 \mathrm{~km} \mathrm{~s}^{-1}\right)$, there appears a weak and isolated emission feature which, however, is rather strong in the $\operatorname{HNCO}\left(5_{0,5}-4_{0,4}\right)$ line. From the channel maps (Fig. 6b), it is clear that this feature peaks at $b \approx-0.15$. The main peak of Clump 2 appears at $\left(l \approx+2.8, v \approx+20 \mathrm{~km} \mathrm{~s}^{-1}\right)$, and the emission is extended to $\left(l \approx+3.2, v \approx+150 \mathrm{~km} \mathrm{~s}^{-1}\right)$. At the so-called forbidden velocities $\left(v_{\mathrm{LSR}}<0 \mathrm{~km} \mathrm{~s}^{-1}\right.$ for $l>0^{\circ}$ and $v_{\mathrm{LSR}}>$ $0 \mathrm{kms}^{-1}$ for $l<0^{\circ}$ ) only little $\mathrm{C}^{18} \mathrm{O}$ emission is visible. Further significant maxima are located at $(l \approx-0.5$, $\left.v \approx-150 \mathrm{kms}^{-1}\right),\left(l \approx+0.1, v \approx-150 \mathrm{~km} \mathrm{~s}^{-1}\right)$, and $\left(l \approx+0.7, v \approx-115 \mathrm{~km} \mathrm{~s}^{-1}\right)$. Note also the $2 \sigma$-emission features at $\left(l \approx-0.5, v \approx+140 \mathrm{~km} \mathrm{~s}^{-1}\right)$ and $(l \approx+1.0$, $\left.v \approx+200 \mathrm{kms}^{-1}\right)$. The $\operatorname{HNCO}\left(5_{0,5}-4_{0,4}\right)$ emission at velocities lower than $-220 \mathrm{~km} \mathrm{~s}^{-1}$ in the $\mathrm{C}^{18} \mathrm{O}$ reference frame will be discussed in Sect. 5 .

\subsubsection{Latitude cuts}

In Fig. 8, we have plotted the intensity for $l$ versus $v_{\text {LSR }}$ at a number of individual latitudes. These $l v$-plots show the spectra (smoothed in velocity as described above) of the $\mathrm{C}^{18} \mathrm{O}(1-0)$ emission in a contour map representation covering the whole longitude range. The levels shown are the intensities of the spectra obtained at a certain latitude multiplied by the latitude spacing of the map. The plotted latitude cuts range from $b=-0.45$ to $b=+0.30$ containing the most interesting features.

At $b=-0.45$ (plot a) in Fig. 8, the emission of local origin showing a double peak in $v$ is the prominent feature (see the description of the $0-\mathrm{km} \mathrm{s}^{-1}$-channel-map in Sect. 4.1.2). It peaks at about $-5 \mathrm{~km} \mathrm{~s}^{-1}$ and $+15 \mathrm{~km} \mathrm{~s}^{-1}$.

The features at $b=-0.30$ (plot b) in Fig. 8 show a rather different shape compared to the integrated plots. Sgr B2 peaks at $-55 \mathrm{~km} \mathrm{~s}^{-1}$ (the $3 \mathrm{kpc}$-arm), and the emission extends from $-130 \mathrm{~km} \mathrm{~s}^{-1}$ to $+65 \mathrm{~km} \mathrm{~s}^{-1}$. In longitude the emission extends to $\mathrm{Sgr} \mathrm{D}$, with a velocity shift to $0 \mathrm{~km} \mathrm{~s}^{-1}$. An inspection of the spectra makes clear that the two weak lines of emission at about $-5 \mathrm{~km} \mathrm{~s}^{-1}$ and $+15 \mathrm{~km} \mathrm{~s}^{-1}$ between Sgr A and B2 are again of local origin. At negative longitudes $\mathrm{Sgr} \mathrm{C}$ is weakly visible, ranging from about $+20 \mathrm{~km} \mathrm{~s}^{-1}$ at $l=-0.5$ to about $-20 \mathrm{~km} \mathrm{~s}^{-1}$ at $l=-0.9$.

At $b=-0.15$ (plot c) in Fig. 8, the emission features as described in Sect. 4.2.1 are present. The more prominent features are the 3-kpc-arm emission, Sgr B1 and B2. Note, that at this latitude the $\operatorname{HNCO}\left(5_{0,5}-4_{0,4}\right)$ emission 
a) Latitude range from -0.90 to +0.75 degree

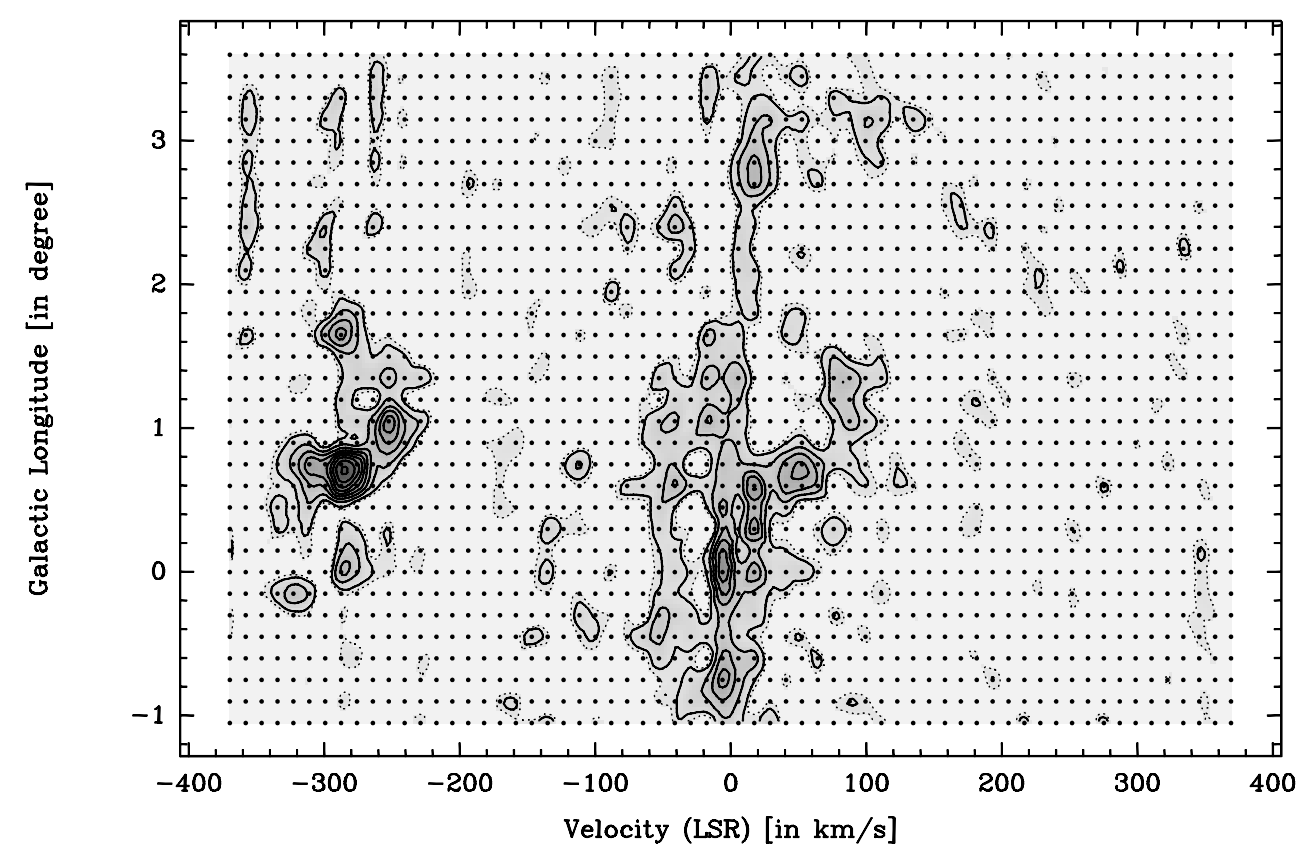

b) Latitude range from -0.30 to +0.30 degree

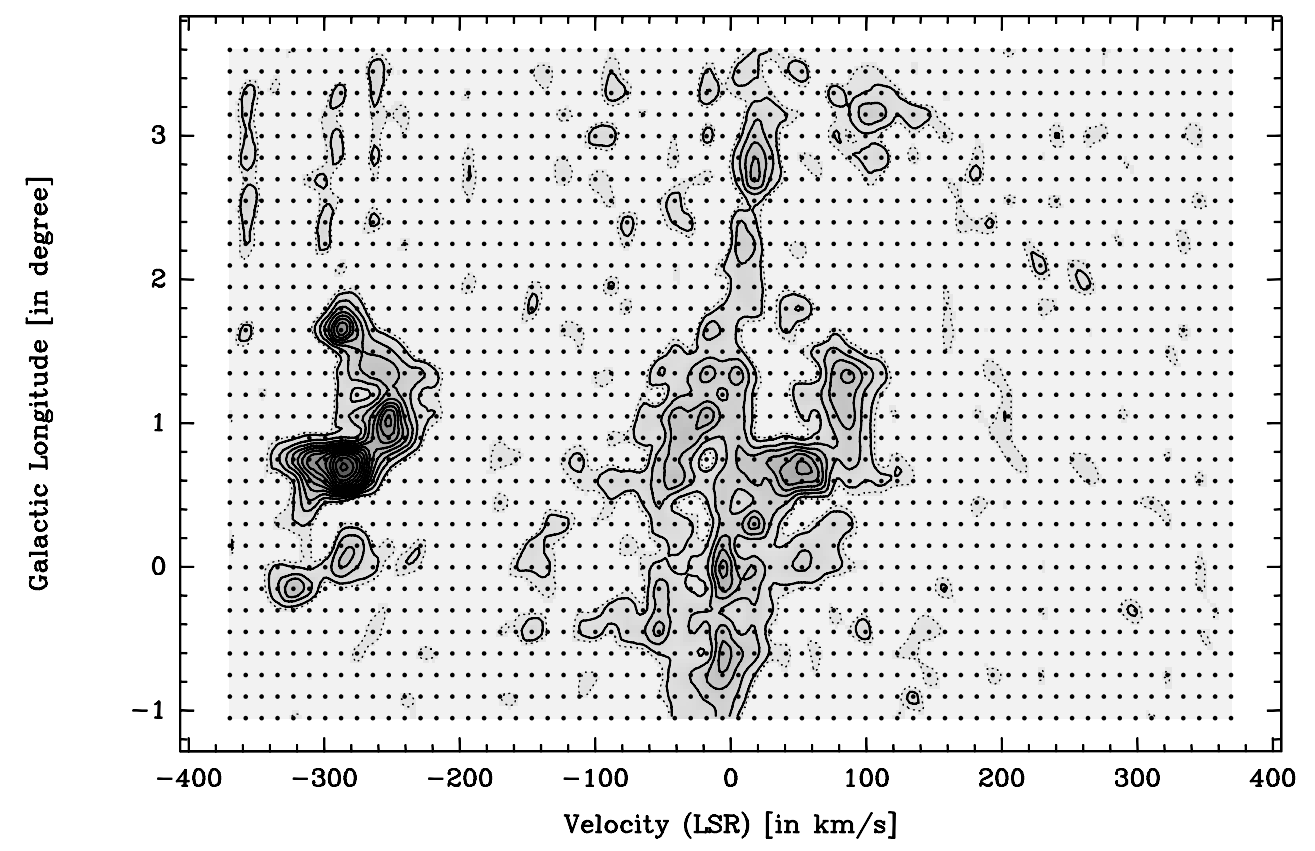

Fig. 7. Longitude-velocity plots showing the velocity distribution of the $\mathrm{C}^{18} \mathrm{O}(1-0)$ emission. Note that the strong emission, which appears at velocities lower than $-200 \mathrm{~km} \mathrm{~s}^{-1}$, is caused by the $\operatorname{HNCO}\left(5_{0,5}-4_{0,4}\right)$ line. a) The velocity distribution in the complete latitude range from -0.90 to $+0^{\circ} .75$. The solid contour levels range from 0.0282 to 0.2506 in steps of $0.0278 \mathrm{~K}$ arcdeg where the lowest level is the $3 \sigma$-value. The dashed contour is at $0.0188 \mathrm{~K}$ arcdeg which is the $2 \sigma$-value. $\mathbf{b}$ ) The velocity distribution in the inner latitude range from -0.30 to +0.30 . The solid contour levels range from 0.018 to 0.222 in steps of $0.017 \mathrm{~K}$ arcdeg where the lowest level is the $3 \sigma$-value. The dashed contour is at $0.012 \mathrm{~K}$ arcdeg which is the $2 \sigma$-value 
a) Latitude: -0.45 degree

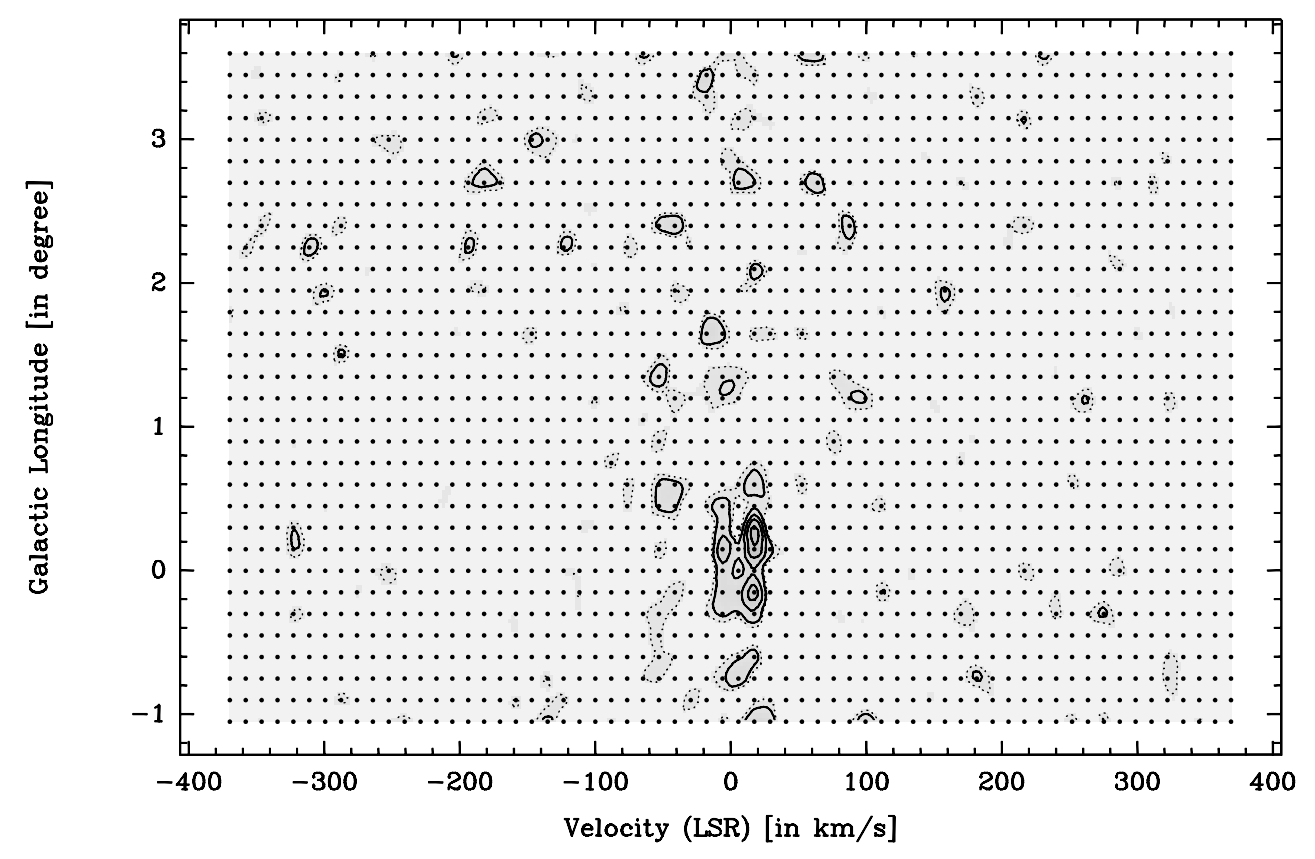

b) Latitude: -0.30 degree

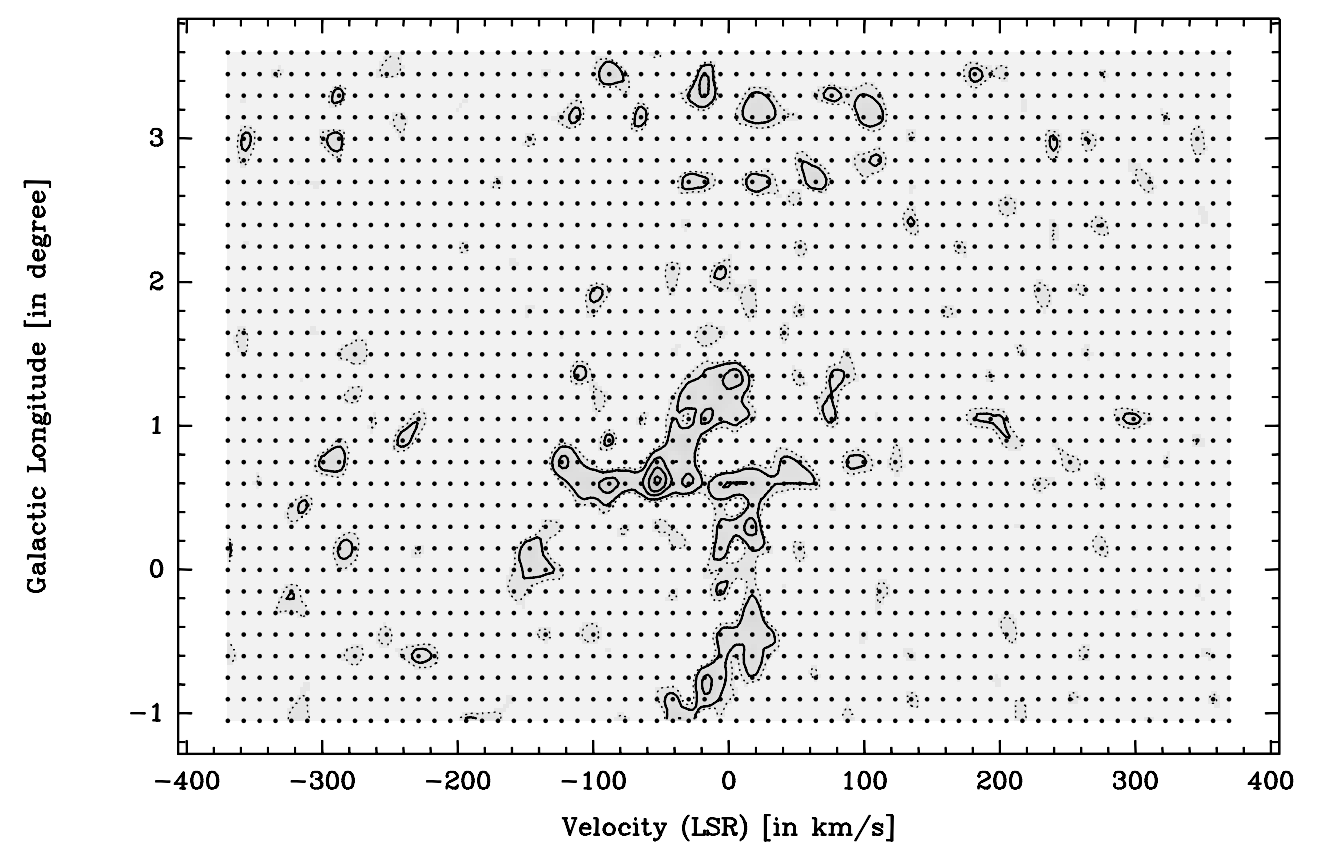

Fig. 8. Longitude-velocity plots of cuts, at single $b$, showing the (smoothed) spectra of the $\mathrm{C}^{18} \mathrm{O}(1-0)$ emission in a contour map representation. The solid contour levels start at $0.0081 \mathrm{~K}$ arcdeg (corresponding to $0.054 \mathrm{~K}$ ), which is the $3 \sigma$-level, and increase in steps of $0.0075 \mathrm{~K}$ arcdeg (corresponding to $0.050 \mathrm{~K}$ ). The dashed contour is at $0.0054 \mathrm{~K}$ arcdeg (corresponding to $0.036 \mathrm{~K}$ ) which is the $2 \sigma$-value. Note that the strong emission, which appears at velocities lower than $-200 \mathrm{~km} \mathrm{~s}^{-1}$, is caused by the $\operatorname{HNCO}\left(5_{0,5}-4_{0,4}\right)$ line. a) In the top panel, the $l v$-plot at $b=-0.45$ is shown. b) In the bottom panel, the $l v$-plot at $b=-0.30$ is shown 

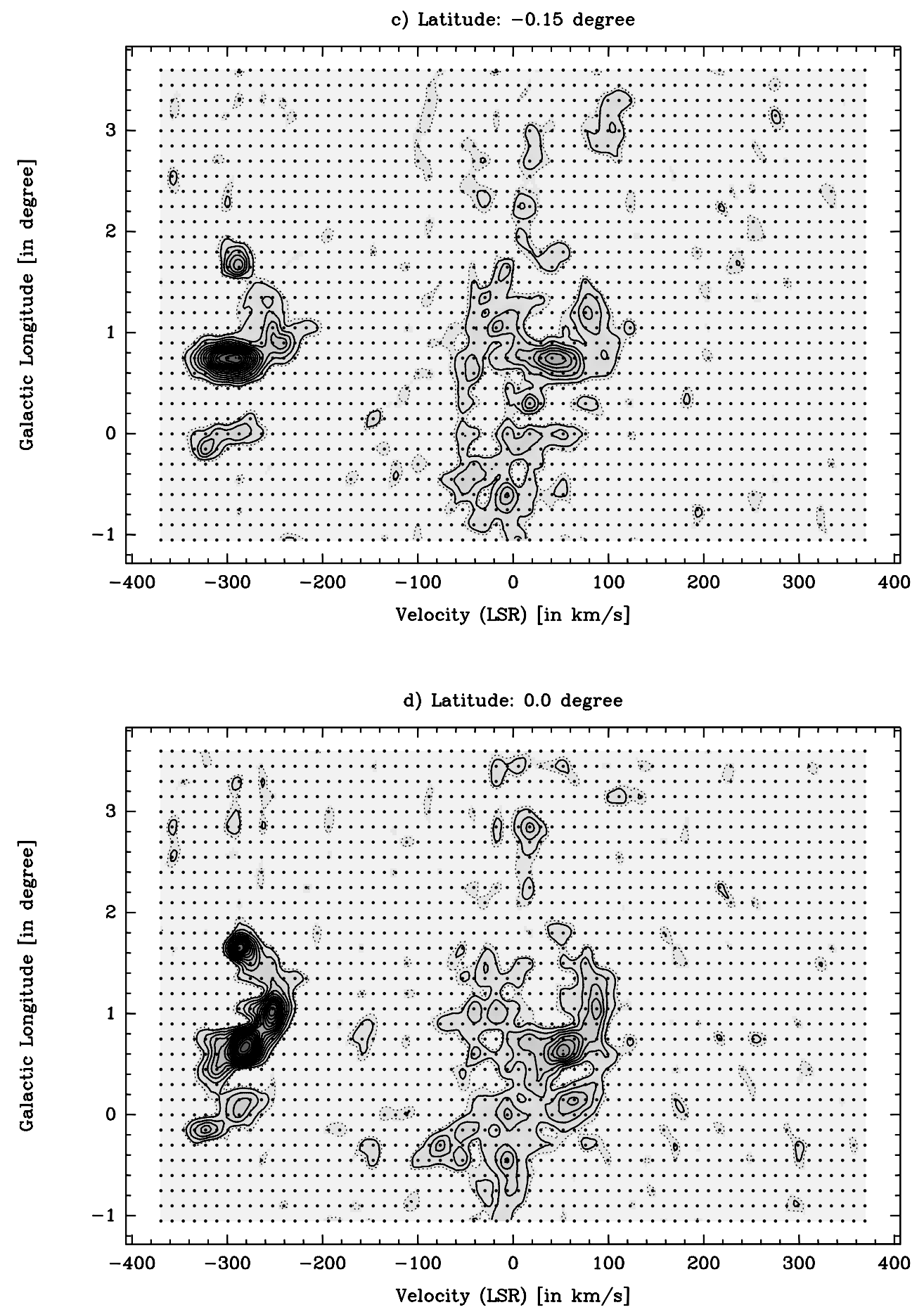

Fig. 8. c) In the top panel, the $l v$-plot of $b=-0^{\circ} 15$ is shown. d) In the bottom panel, the $l v$-plot of $b=0.0$ is shown

appears abruptly whereas it is not visible at more negative latitudes.

At $b=0.0$ (plot d) in Fig. 8, the emission features as described in Sect. 4.2.1 are again present. At this latitude the more prominent features are the emission line at $-5 \mathrm{~km} \mathrm{~s}^{-1}$ ranging from $l=-0.9$ to $l=+1.5$, the diagonal chain of emission centers ranging from $\mathrm{Sgr} C$ to Sgr A to Sgr B2 and further to Sgr D, and the emission 

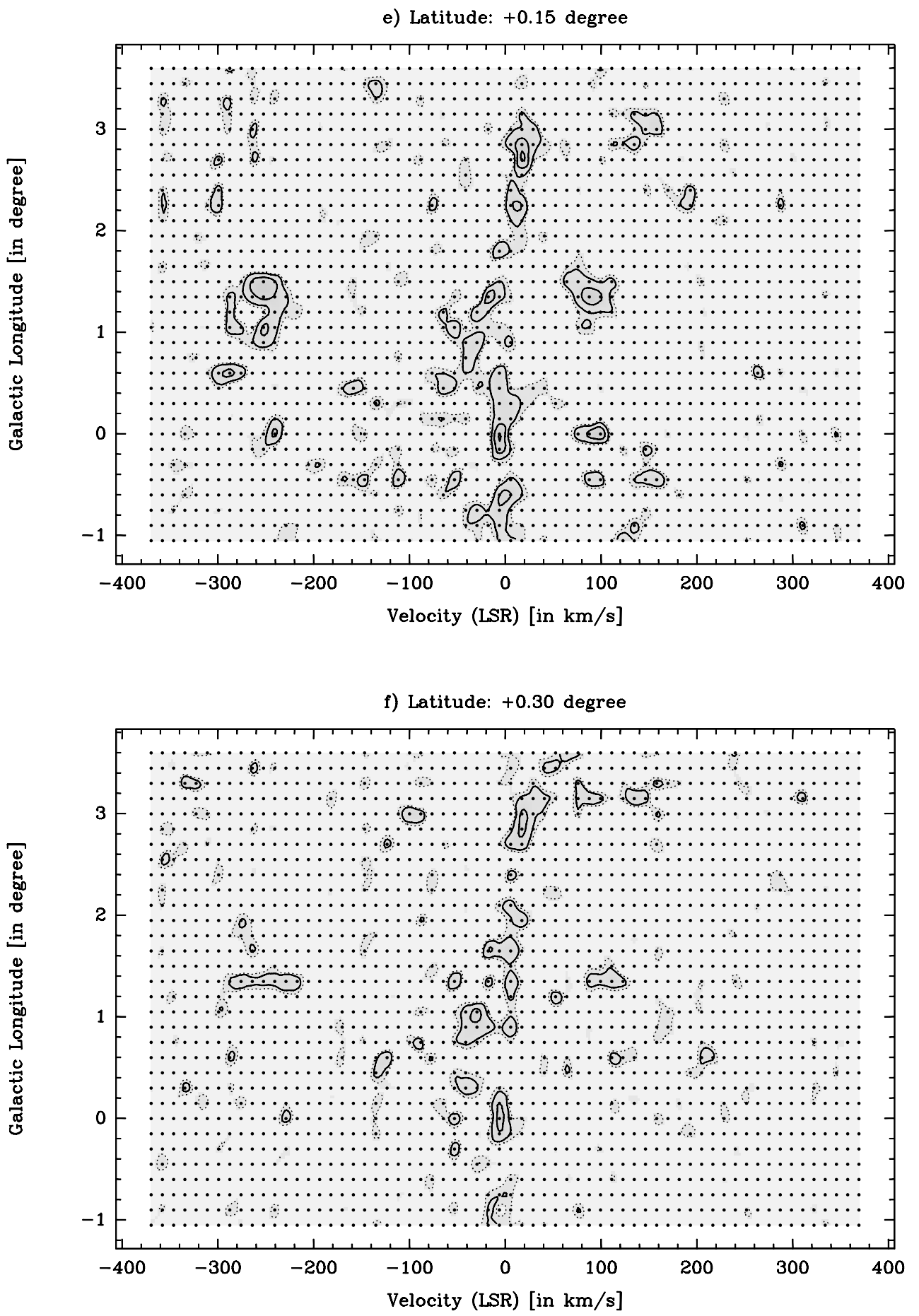

Fig. 8. e) In the top panel, the $l v$-plot of $b=+0^{\circ} 15$ is shown. f) In the bottom panel, the $l v$-plot of $b=+0^{\circ} 30$ is shown

associated with Sgr A extended to $+95 \mathrm{~km} \mathrm{~s}^{-1}$. Note that at this latitude Sgr B1 does not appear in this chain. The presence of this feature in the plot at $b=-0.15$ and its absence at $b=0.0$ cannot be distinguished in the plot integrated over a range of latitudes.

At $b=+0.15$ (plot e) in Fig. 8, the emission line at $-5 \mathrm{~km} \mathrm{~s}^{-1}$ is visible, including the velocity shift at +1.8 

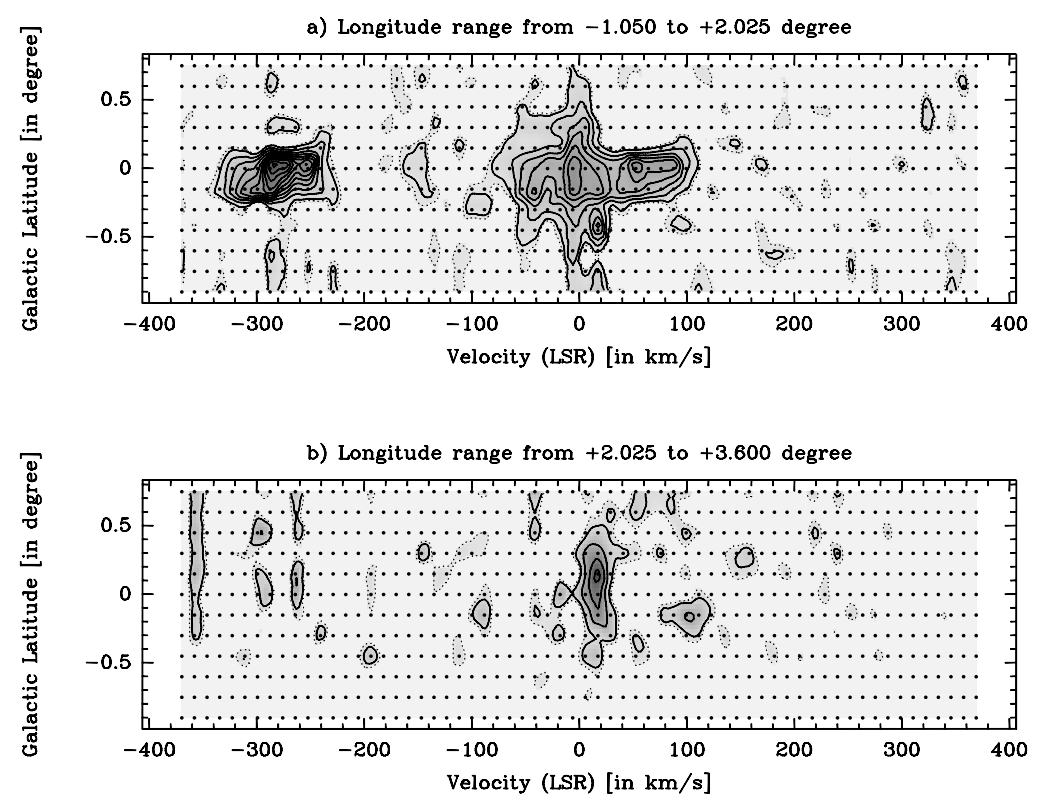

Fig. 9. Latitude-velocity plots showing the velocity distribution of the $\mathrm{C}^{18} \mathrm{O}(1-0)$ emission. a) The velocity distribution in the longitude range from -1.05 to +2.025 which represents the inner Galactic center region. The solid contour levels range from 0.039 to 0.429 in steps of $0.039 \mathrm{~K}$ arcdeg where the lowest level is the $3 \sigma$-value. The dashed contour is at $0.026 \mathrm{~K}$ arcdeg which is the $2 \sigma$-value. $\mathbf{b})$ The velocity distribution in the longitude range from +2.025 to +3.60 which represents Clump 2 . The solid contour levels range from 0.027 to 0.108 in steps of $0.027 \mathrm{~K}$ arcdeg where the lowest level is the $3 \sigma$-value. The dashed contour is at $0.018 \mathrm{~K}$ arcdeg which is the $2 \sigma$-value

to roughly $+10 \mathrm{kms}^{-1}$ and the continuation to +3.1 at about $+20 \mathrm{kms}^{-1}$. In addition, Sgr D shows an isolated feature at about $+95 \mathrm{~km} \mathrm{~s}^{-1}$ and a loop-like structure that seems to connect its zero velocity emission to the zero velocity emission of Sgr B2, passing over $-40 \mathrm{~km} \mathrm{~s}^{-1}$ at $l=0.9$. Clump 2 has an additional emission feature at about $+150 \mathrm{~km} \mathrm{~s}^{-1}$. Note that at higher latitudes than $b=$ 0.0 the $\operatorname{HNCO}\left(5_{0,5}-4_{0,4}\right)$ emission disappears abruptly; only at Sgr D some emission appears at those latitudes.

The emission at $b=+0^{\circ} 30$ (plot f) in Fig. 8 looks similar to that at $b=+0.15$, except that it is somewhat weaker.

\subsection{Latitude-velocity plots}

In Fig. 9, we have plotted the intensity integrated over two longitude ranges. These latitude-velocity plots ( $b v$ plots) show the velocity distribution of the $\mathrm{C}^{18} \mathrm{O}(1-0)$ emission a) in the longitude range from -1.05 to +2.025 , which represents the inner Galactic center region, and b) in the longitude range from +2.025 to +3.60 which represents Clump 2. The averaging of velocity channels, data interpolation and determination of the $1 \sigma$-level were done following the procedures used for the $l v$-plots.

Plot a) in Fig. 9 displays the inner Galactic center region. It shows a cross-shaped emission feature in
$\mathrm{C}^{18} \mathrm{O}(1-0)$. The horizontal bar follows the $b=0^{\circ}$ line in the range from $-80 \mathrm{~km} \mathrm{~s}^{-1}$ to $+110 \mathrm{~km} \mathrm{~s}^{-1}$. Sgr C, A, and $\mathrm{B} 2$, respectively, can be seen as peaks at $-40,-5$, and $+55 \mathrm{~km} \mathrm{~s}^{-1}$. The extension toward $+110 \mathrm{~km} \mathrm{~s}^{-1}$ represents Sgr D. The vertical bar of the cross toward negative latitude seems to represent gas of local origin. It is partly due to the double-peaked local gas feature already discussed in Sect. 4.2 .2 (plots a) and b) in Fig. 8. But in general this vertical bar shows the larger $b$-extension of the $\mathrm{C}^{18} \mathrm{O}(1-0)$ emission around $v=0 \mathrm{~km} \mathrm{~s}^{-1}$ (see Fig. $6 \mathrm{~b}$ ).

In Plot b) of Fig. 9 mainly Clump 2 is visible. The emission shows the large extent of Clump 2 to positive latitudes at about $+15 \mathrm{~km} \mathrm{~s}^{-1}$. In addition, the higher velocity component of Clump 2 becomes visible near $(b=$ $\left.-0.15, v=+105 \mathrm{~km} \mathrm{~s}^{-1}\right)$.

\section{The $\mathrm{HNCO}\left(5_{0,5}-4_{0,4}\right)$ line in the survey}

Because of the large bandwidth of the AOS, covering $\pm 375 \mathrm{~km} \mathrm{~s}^{-1}$, the $5_{0,5}-4_{0,4}$ transition of isocyanic acid (HNCO) at $109.905573 \mathrm{GHz}$ (Lovas 1992), which corresponds to a velocity shift of $-337.5 \mathrm{~km} \mathrm{~s}^{-1}$ with respect to the $\mathrm{C}^{18} \mathrm{O}(1-0)$ line frequency, fell into the range of the spectrometer as long as the $v_{\mathrm{LSR}}$ of the emission was not very negative. 


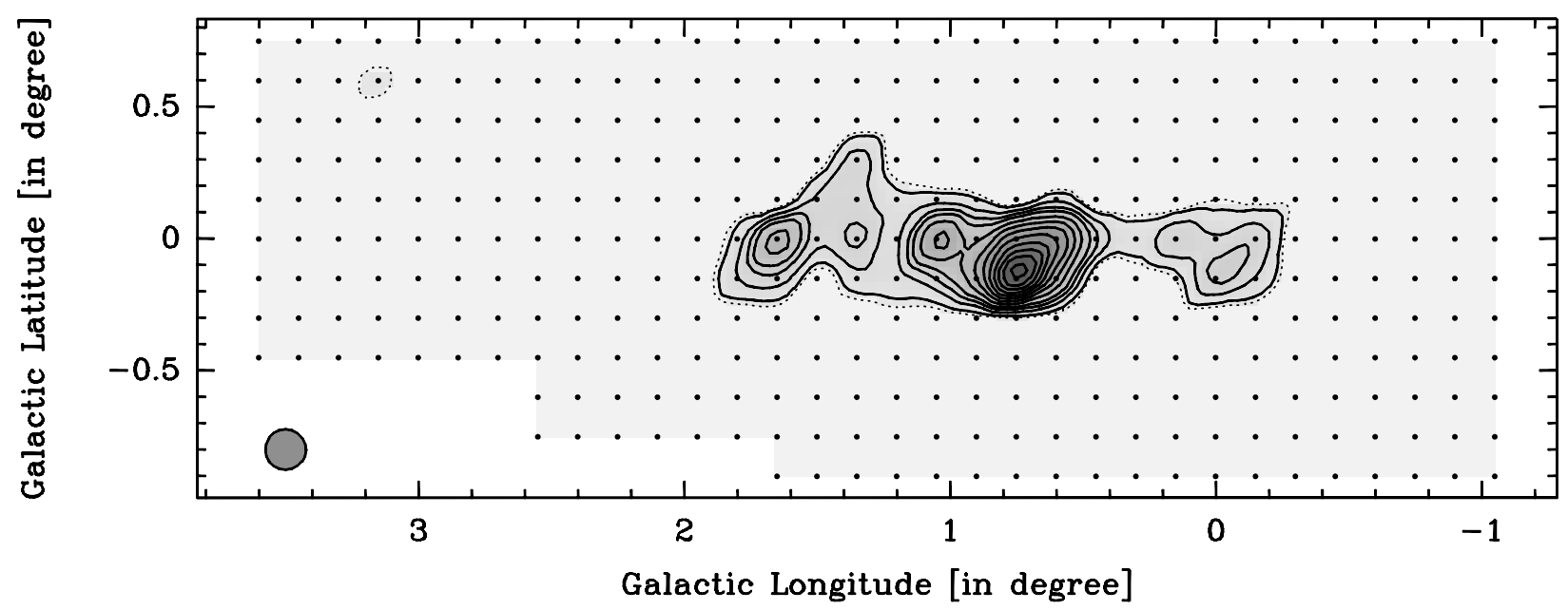

Fig. 10. The integrated intensity of the Galactic center region in $\operatorname{HNCO}\left(5_{0,5}-4_{0,4}\right)$. The velocity over which the intensity is integrated ranges from -37.5 to $+137.5 \mathrm{~km} \mathrm{~s}^{-1}$. The solid contour levels are 2.4, 5.0, 7.5, and from 10.0 to 42.0 in steps of $4.0 \mathrm{~K} \mathrm{~km} \mathrm{~s}^{-1}$ where the lowest level is the $3 \sigma$-value. The dashed contour is at $1.6 \mathrm{~K} \mathrm{~km} \mathrm{~s}^{-1}$ which is the $2 \sigma$-value. The circle in the lower left corner of the plot indicates the beam size of 9 '.2

\subsection{Introductory notes on $\mathrm{HNCO}$}

HNCO is a molecule with a slight asymmetry caused by the protrusion of the hydrogen atom and it has an unusual energy structure: The $K_{-1}>0$ ladders of rotational levels lie tens of degrees $\mathrm{K}$ above the corresponding levels in the $K_{-1}=0$ ladder. Hence, nearly all HNCO should be in states of the $K_{-1}=0$ ladder if the molecule is collisionally excited. In this case, the two level model of Jackson et al. (1984) indicates that the $\mathrm{H}_{2}$ density has to be at least $310^{4} \mathrm{~cm}^{-3}$ and is typically $\gtrsim 10^{6} \mathrm{~cm}^{-3}$. Thus, if excited collisionally HNCO is a very high density tracer. On the other hand, the lowest $K_{-1}=1$ levels are separated from the lowest $K_{-1}=0$ levels by energies which correspond to far-infrared (FIR) wavelengths. Thus, a significant population in the $K_{-1}>0$ levels is a clear indication of radiative instead of collisional excitation. See Winnewisser et al. (1976) for a detailed study of the molecular parameters of $\mathrm{HNCO}$.

HNCO was first detected in Sgr B2 (Snyder \& Buhl 1972). Subsequent observations in the Galactic disk (Jackson et al. 1984) show the $5_{0,5}-4_{0,4}$ transition to be distinctly weaker than the $\mathrm{C}^{18} \mathrm{O}(1-0)$ line. The abundance found for disk clouds roughly agrees with chemical models (Iglesias 1977; Huntress \& Mitchell 1979), whereas the emission in Sgr A (Armstrong \& Barrett 1985) and Sgr B2 (Churchwell et al. 1986; Cummins et al. 1986) is more than an order of magnitude stronger than expected. At least in Sgr B2, HNCO seems to be mainly excited by FIR radiation.
Throughout the Galactic center, there is, however, a large variation in the $\operatorname{HNCO}\left(5_{0,5}-4_{0,4}\right) / \mathrm{C}^{18} \mathrm{O}(1-0)$ ratio. From a sample of 34 clouds in the inner $500 \mathrm{pc}$ of the Galactic center region, Hüttemeister (1993), Chapter 5 , found both low values, $<0.2$, for this intensity ratio, similar to those found for the Galactic Disk by Jackson et al. (1984), and high values of 3 to 5, as found in Sgr A by Jackson et al. In addition, Hüttemeister (1993) could not find any correlation between clouds with strong HNCO emission and IRAS point sources which might be a possible indicator for the presence of strong (local) FIR radiation.

\subsection{Presentation of the $\mathrm{HNCO}$ data}

For the full set of survey spectra refer to the presentation of the $\mathrm{C}^{18} \mathrm{O}$ spectra in Fig. 4 of Sect. 4. These spectra includes the complete bandwidth of the backend so that the $\operatorname{HNCO}\left(5_{0,5}-4_{0,4}\right)$ line is also visible (with the mentioned shift in the velocity scale of $\left.-337.5 \mathrm{~km} \mathrm{~s}^{-1}\right)$.

\subsubsection{The integrated intensity}

In Fig. 10, the integrated intensity is plotted as a contour map, covering the velocity range from -37.5 to $+137.5 \mathrm{~km} \mathrm{~s}^{-1}$. This is the complete emission range of the $\operatorname{HNCO}\left(5_{0,5}-4_{0,4}\right)$ line which is covered by the spectra. Toward negative velocities this is limited by the edge of the spectrometer. Toward positive velocities there is no confusion with the $\mathrm{C}^{18} \mathrm{O}$ emission, as can be seen in the 

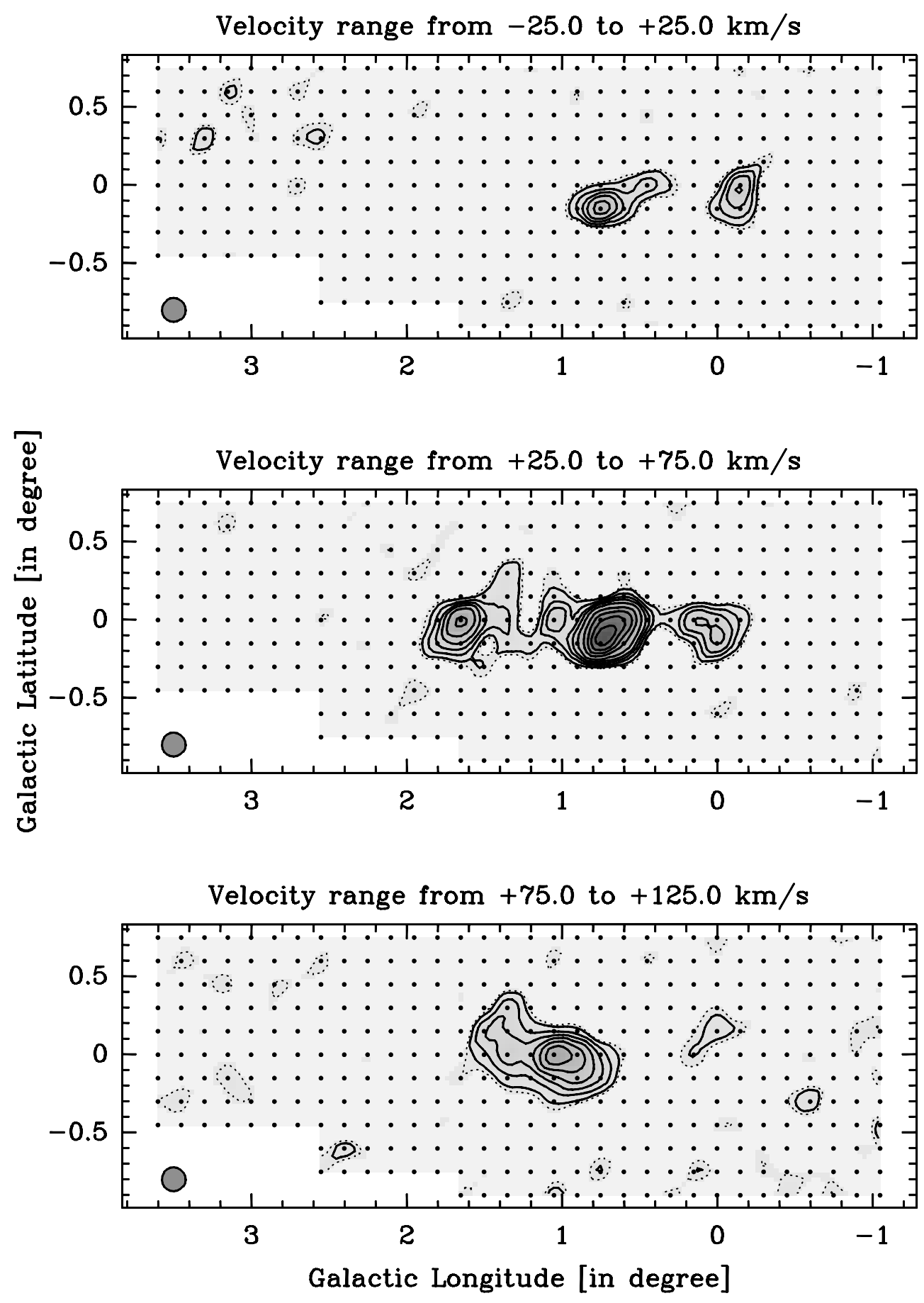

Fig. 11. The integrated intensity of the Galactic center region, in $\operatorname{HNCO}\left(5_{0,5}-4_{0,4}\right)$, in velocity intervals of $50 \mathrm{~km} \mathrm{~s}^{-1}$ width. The solid contour levels start at $1.3 \mathrm{~K} \mathrm{~km} \mathrm{~s}^{-1}$, which is the $3 \sigma$-level, continue with $2.7,4.2,6.0$, and $9.0 \mathrm{~K} \mathrm{~km} \mathrm{~s}^{-1}$ and then increase in steps of $5.0 \mathrm{~K} \mathrm{~km} \mathrm{~s}^{-1}$. The dashed contour is at $0.8667 \mathrm{~K} \mathrm{~km} \mathrm{~s}^{-1}$ which is the $2 \sigma$-value. The circle in the lower left corner of the plots indicates the beam size of 9.2. In the top panel the integrated intensity of the velocity range from -25.0 to $+25.0 \mathrm{~km} \mathrm{~s}^{-1}$ is plotted, in the middle panel the velocity ranges from +25.0 to $+75.0 \mathrm{~km} \mathrm{~s}^{-1}$, and at the bottom the velocity range from +75 to $+125.0 \mathrm{~km} \mathrm{~s}^{-1}$ is displayed 
$l v$-plots, in particular in the latitude cuts, of $\mathrm{C}^{18} \mathrm{O}(1-0)$ (see Figs. 7 and 8 ) where no $\operatorname{HNCO}\left(5_{0,5}-4_{0,4}\right)$ emission appears at velocities higher than $+137.5 \mathrm{~km} \mathrm{~s}^{-1}$ (which corresponds to $-200 \mathrm{~km} \mathrm{~s}^{-1}$ in $\mathrm{C}^{18} \mathrm{O}$ ). Apart from the mentioned differences, Fig. 10 is produced in the same way as Fig. 5.

Most notably, the $\operatorname{HNCO}\left(5_{0,5}-4_{0,4}\right)$ emission is much more restricted to the Galactic plane than the $\mathrm{C}^{18} \mathrm{O}(1-0)$ emission. In general, it appears abruptly at $b=-0.15$ but is not visible at more negative latitudes. Similarly, it disappears abruptly at latitudes more positive than $b=0.0$; only in the area of Sgr D some emission appears at higher latitudes. The strongest source by far is Sgr B2. In relation to the other sources, Sgr B2 is even more prominent in $\mathrm{HNCO}$ than in $\mathrm{C}^{18} \mathrm{O}$. It is not clear to what degree systematic effects at the edge of the spectrometer hide emission features. Therefore, even though no emission at negative velocities was found, some emission might be present. This could be the reason for the seemingly complete absence of Sgr C and could also have weakened the emission of Sgr A. In addition, this could, at least partly, be the reason for the smaller $b$-extension of $\mathrm{HNCO}$; as one can see from Fig. 6 , the $b$-extension of $\mathrm{C}^{18} \mathrm{O}(1-0)$ at negative velocities is larger than at positive velocities. Similar to $\mathrm{C}^{18} \mathrm{O}$, Sgr D (the $l=1.5$-complex of Bally et al. 1988) peaks at about $(l=1.0, b=0.0)$ and has an arc-like extension to $(l=1.35, b=+0.4)$. In the area of $l=1.5$ to 1.85 and $b=-0.25$ to +0 . 1 , there appears a fairly strong emission feature which peaks at $(l=1.65, b=0.0)$. This feature - hereafter called HNCO-1.65-0.0 - is rather interesting because it has only a very weak and barely detected counterpart in $\mathrm{C}^{18} \mathrm{O}$ peaking at $(l=1.75, b=-0.15)$. Especially at the peak position of the HNCO emission, no $\mathrm{C}^{18} \mathrm{O}$ is visible.

In Fig. 11, the integrated intensity of the $\operatorname{HNCO}\left(5_{0,5}-4_{0,4}\right)$ line is plotted in velocity intervals of $50 \mathrm{~km} \mathrm{~s}^{-1}$ width. Because of the limitation in velocity due to the edge of the spectrometer band toward negative velocities and due to the lack of emission at higher positive velocities only the channels of $0 \mathrm{~km} \mathrm{~s}^{-1},+50 \mathrm{~km} \mathrm{~s}^{-1}$, and $+100 \mathrm{~km} \mathrm{~s}^{-1}$ are plotted. Apart from that, the plots are produced as in Fig. 6 .

At $0 \mathrm{~km} \mathrm{~s}^{-1}$, only little $\mathrm{HNCO}$ is visible, in contrast to $\mathrm{C}^{18} \mathrm{O}$ which is widespread and uniformly distributed at this velocity. However, distinct but limited emission regions are visible at $\operatorname{Sgr} \mathrm{A}(l=0.0, b=0.0)$ and at $(l=0.75, b=-0.15)$ somewhat east of Sgr B2. The latter region has an extension to $(l=0.5, b=0.0)$. These regions coincide with the strongest emission regions in $\mathrm{C}^{18} \mathrm{O}$. Because of the reasons mentioned above, the emission from Sgr C and a larger $b$-extension might be systematically suppressed. However, this cannot explain the more limited extension in $l$ of the HNCO emission at this velocity, because the emission at higher positive longitudes tends to have positive velocities. Thus, this point supports the idea that $\mathrm{HNCO}$ is more restricted to limited areas than $\mathrm{C}^{18} \mathrm{O}$. Emission related to Clump 2 seems to appear in some isolated spots, but it seems possible that these features which are just barely rising above the $3 \sigma$ level are introduced by effects caused by the edge of the backend.

At $50 \mathrm{kms}^{-1}, \mathrm{Sgr} \mathrm{B} 2$ is, as in $\mathrm{C}^{18} \mathrm{O}$, by far the most prominent emission feature. It peaks at $(l=0.7, b=$ -0.1 ) which is somewhat (but not significantly) further to the southeast than in the respective $\mathrm{C}^{18} \mathrm{O}$ plot (Fig. 6). The integrated intensity of more than $30 \mathrm{~K} \mathrm{~km} \mathrm{~s}^{-1}$ at this peak position is twice as high as in the $\mathrm{C}^{18} \mathrm{O}$ plot. The second strongest source is HNCO-1.65-0.0 which appears only within this velocity interval. As in the $\mathrm{C}^{18} \mathrm{O}$ plot, Sgr A and D are present with clear emission signatures, but in comparison to Sgr B2, they seem rather weak. In addition, the eastern part of Sgr D is missing due to an emission free valley at $l=1.2$. Sgr C is not visible, but weak emission could be hidden due to effects caused by the edge of the backend. In contrast to $\mathrm{C}^{18} \mathrm{O}$, Clump 2 is not visible in HNCO.

At $100 \mathrm{~km} \mathrm{~s}^{-1}, \operatorname{Sgr} \mathrm{D}$ with the arc-like extension $(l=$ 1.5 -complex $)$ to $(l=1.35, b=+0.4)$ is, as in $\mathrm{C}^{18} \mathrm{O}$, the dominant feature. The shape and extension are nearly the same as in $\mathrm{C}^{18} \mathrm{O}$, only the extension toward $\mathrm{Sgr} \mathrm{B} 2$ is absent. Also as in $\mathrm{C}^{18} \mathrm{O}$, emission is visible slightly north of Sgr A. In contrast to $\mathrm{C}^{18} \mathrm{O}$, Clump 2 is not visible in HNCO.

\subsubsection{Longitude-velocity plots}

In Fig. 12, we have plotted the intensity integrated over two latitude ranges showing the velocity distribution of the $\operatorname{HNCO}\left(5_{0,5}-4_{0,4}\right)$ emission. In part a) of the figure, the latitude ranges from -0.30 to +0.30 covering the complete $b$-extension of the $\mathrm{HNCO}$ emission. In part b) of the figure, the latitude integral from -0.15 to +0 . 15 , which covers the major HNCO emission area, is shown.

In general, both plots show the same features. Because the HNCO emission is concentrated toward the plane, the emission features are more prominent in plot b). There is no emission near $v_{\text {LSR }}=0 \mathrm{~km} \mathrm{~s}^{-1}$. The 3 -kpcarm (Rougoor \& Oort 1960) is beyond the edge of the spectrometer band. The diagonal chain of emission centers which is very prominent in $\mathrm{C}^{18} \mathrm{O}$ is only represented by Sgr A, Sgr B2, and Sgr D. In HNCO, this feature resembles a spiral, starting in the weak emission extending from $\operatorname{Sgr} A$ to $\left(l \approx+0.05, v \approx+90 \mathrm{~km} \mathrm{~s}^{-1}\right)$, continuing through Sgr A itself with a peak at $(l \approx-0.15$, $\left.v \approx+15 \mathrm{~km} \mathrm{~s}^{-1}\right)$, turning toward positive longitudes and velocities for $\operatorname{SgrB} 2\left(l \approx+0.7, v \approx+55 \mathrm{~km} \mathrm{~s}^{-1}\right)$, to $\operatorname{Sgr} \mathrm{D}\left(l \approx+1.0, v \approx+85 \mathrm{~km} \mathrm{~s}^{-1}\right)$, and turning back toward lower velocities for HNCO-1.65-0.0 at $(l \approx+1.65$, $\left.v \approx+50 \mathrm{~km} \mathrm{~s}^{-1}\right)$. The lines of weak emission which appear at $-20 \mathrm{~km} \mathrm{~s}^{-1},+40 \mathrm{~km} \mathrm{~s}^{-1}$, and $+75 \mathrm{~km} \mathrm{~s}^{-1}$ in the longitude range from 2.25 to 3.50 (Clump 2 area) are dubious because these are visible neither in the contour plot 
a) Latitude range from -0.30 to +0.30 degree

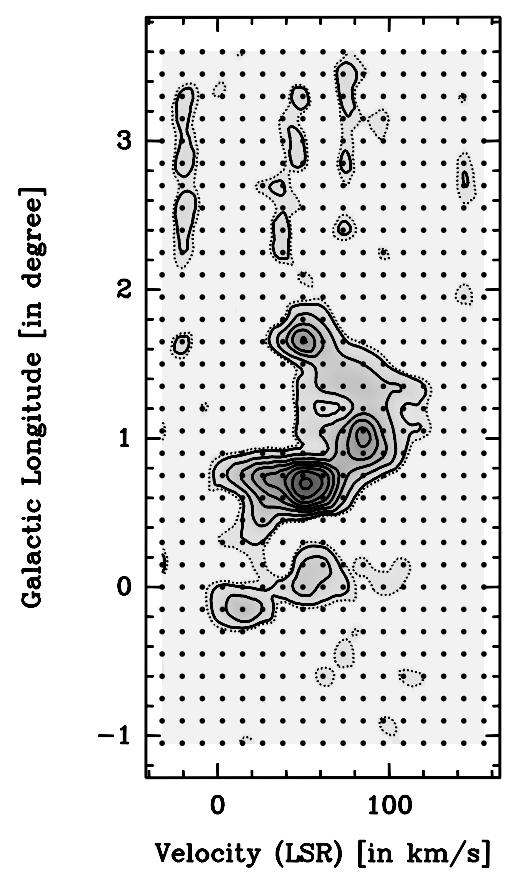

b) Latitude range from -0.15 to +0.15 degree

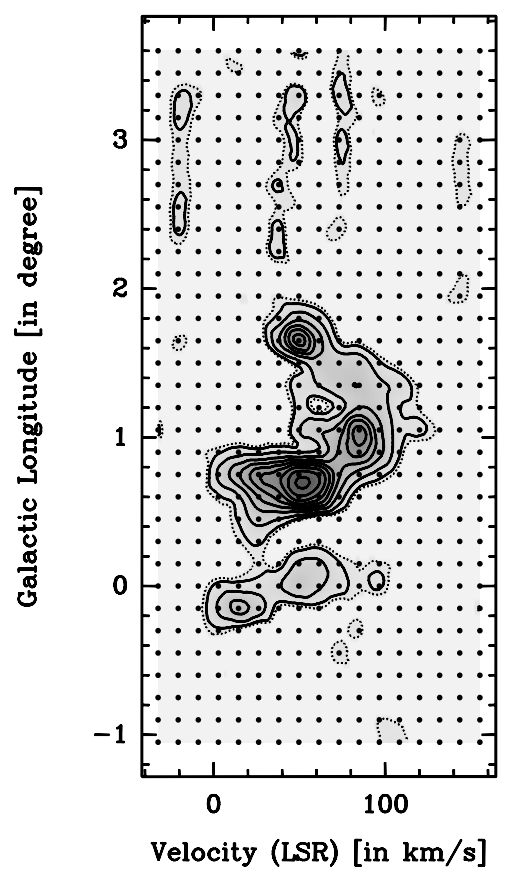

Fig. 12. Longitude-velocity plots showing the velocity distribution of the $\operatorname{HNCO}\left(5_{0,5}-4_{0,4}\right)$ emission. a) The velocity distribution in the latitude range from $-0^{\circ} 30$ to $+0^{\circ} 30$ which covers the complete HNCO emission area. The solid contour levels range from $0.018,0.04$ to 0.22 in steps of $0.03 \mathrm{~K}$ arcdeg where the lowest level is the $3 \sigma$-value. The dashed contour is at $0.012 \mathrm{~K}$ arcdeg which is the $2 \sigma$-value. b) The velocity distribution in the latitude range from -0.15 to $+0^{\circ} .15$ which covers the major $\mathrm{HNCO}$ emission area. The solid contour levels range from $0.0141,0.03$ to 0.205 in steps of $0.025 \mathrm{~K}$ arcdeg where the lowest level is the $3 \sigma$-value. The dashed contour is at $0.0094 \mathrm{~K}$ arcdeg which is the $2 \sigma$-value

of the total integrated intensity (Fig. 10) nor in the channel maps (Fig. 11). In addition, such streamers of emission at these velocities are not found for any other molecular line.

In Fig. 13, we show the intensity at selected latitudes, analogous to Fig. 6 . The latitude cuts range from $b=$ -0.15 to $b=+0.30$ covering the most interesting features.

At $b=-0$. 15 , the emission features show similarity to the features integrated over latitude in Fig. 12 but some distinct deviations are visible. Sgr B2 appears as a riftlike ellipsoidal maximum at $l=+0.75$, with $v$ ranging from $+30 \mathrm{~km} \mathrm{~s}^{-1}$ to $+50 \mathrm{~km} \mathrm{~s}^{-1}$. There is no connection between Sgr B2 and the Sgr A features. The maximum of Sgr $\mathrm{D}$ is shifted to $\left(l \approx+0.9, v \approx+90 \mathrm{~km} \mathrm{~s}^{-1}\right)$, and the connection to HNCO-1.65-0.0 is rather diffuse and only barely tracing a spiral.

At $b=0.0$, the emission features are very close to what is visible in Fig. 12 but more prominent. Especially the spiral structure is more emphasized, and it is clear from this plot that the Sgr A emission at $(l \approx-0.15$, $\left.v \approx+15 \mathrm{~km} \mathrm{~s}^{-1}\right)$ is not part of the spiral-like structure in the $l v$-space.

At $b=+0$. 15 , nearly all $\operatorname{HNCO}\left(5_{0,5}-4_{0,4}\right)$ emission has disappeared. Only in the area near Sgr D (the 1.5-complex) there is some weak but widespread emission present. In addition, Sgr B2 is marginally visible.

At $b=+0.30$, almost no HNCO emission is present. It is not clear if the extended line of emission at $l=+1.35$, ranging in $v$ from $+50 \mathrm{~km} \mathrm{~s}^{-1}$ to $+120 \mathrm{~km} \mathrm{~s}^{-1}$, is real but there is no obvious reason to doubt it. In addition, a weak counterpart in $\mathrm{C}^{18} \mathrm{O}$ exists.

\section{6. ${ }^{12} \mathrm{CO}(1-0)$ measurements}

One goal of the $\mathrm{C}^{18} \mathrm{O}(1-0)$ Galactic Center Survey is a comparison with other isotopomers of $\mathrm{CO}$, especially with ${ }^{12} \mathrm{CO}$. For this purpose it is necessary to determine the comparability of the data, i.e., any positional or other misalignment of our data compared to data from other telescope systems. Because no large scale $\mathrm{C}^{18} \mathrm{O}(1-0)$ survey of the Galactic center region was available up to now, it was decided to observe the Galactic center region in ${ }^{12} \mathrm{CO}(1-0)$ using the same system. The last widely published large scale ${ }^{12} \mathrm{CO}(1-0)$ data of the Galactic center (Liszt \& Burton 1978; Bania 1977, 1980, 1986) are more than 20 years old and not very sensitive. However, there exist more recent data, one set observed in 1984 with the 1.2 m SMWT (Bitran 1987; Bitran et al. 1997), 
a) Latitude: -0.15 degree

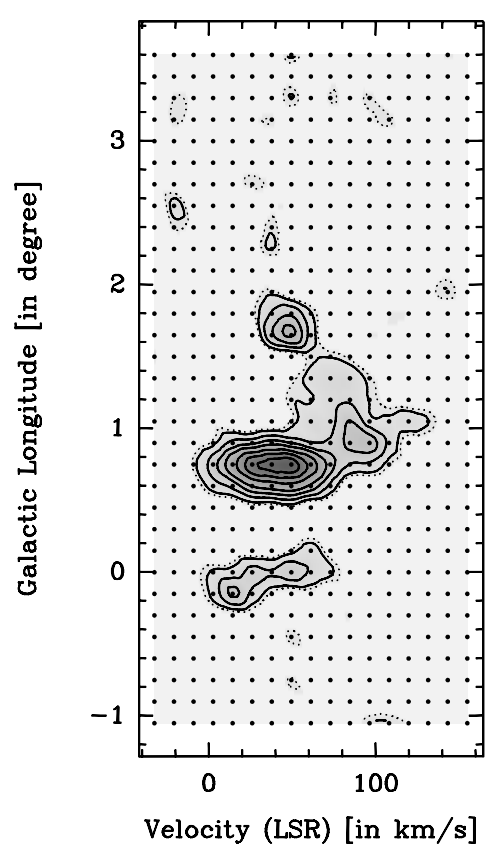

c) Latitude: +0.15 degree

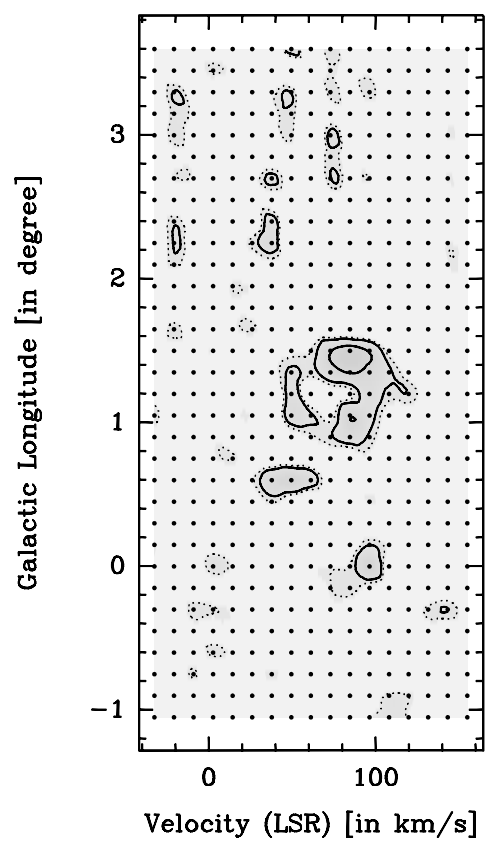

b) Latitude: 0.0 degree

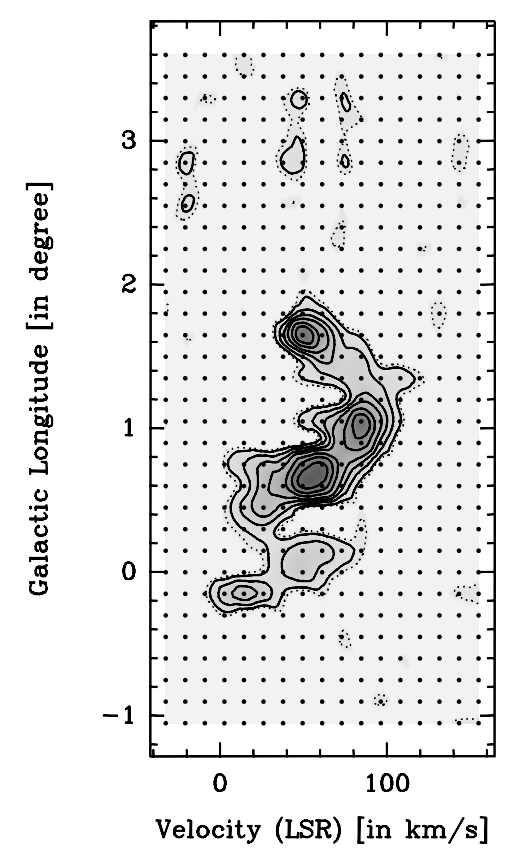

d) Latitude: +0.30 degree

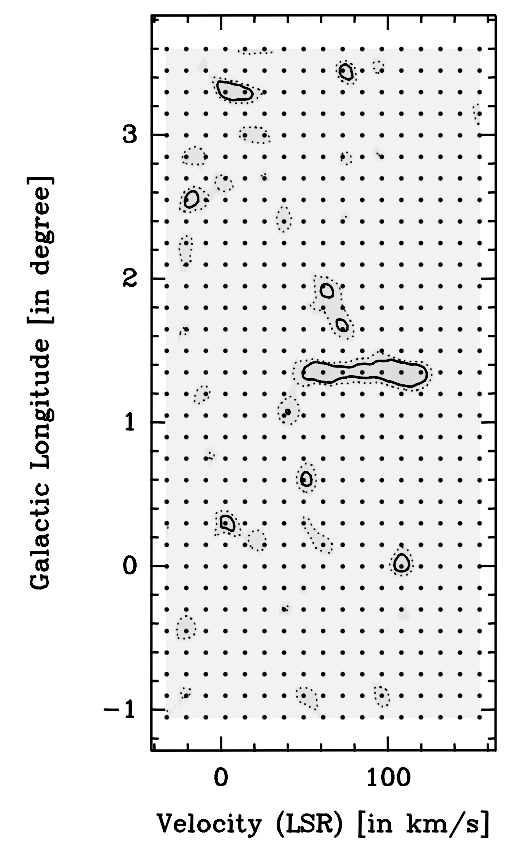

Fig. 13. Longitude-velocity plots of cuts at single $b$ showing the (smoothed) spectra of the $\operatorname{HNCO}\left(5_{0,5}-4_{0,4}\right)$ emission in a contour map representation. The solid contour levels start at $0.0081 \mathrm{~K}$ arcdeg (corresponding to $0.054 \mathrm{~K}$ ), which is the $3 \sigma$-level, continue with 0.017 and $0.028 \mathrm{~K}$ arcdeg, and then increase from $0.04 \mathrm{~K}$ arcdeg in steps of $0.02 \mathrm{~K}$ arcdeg. The dashed contour is at $0.0054 \mathrm{~K}$ arcdeg (corresponding to $0.036 \mathrm{~K}$ ) which is the $2 \sigma$-value. a) In the upper left panel, the $l v$-plot at $b=-0.15$ is shown. b) In the upper right panel, the $l v$-plot at $b=0.0$ is shown. c) In the lower left panel, the $l v$-plot at $b=+0.15$ is shown. d) In the lower right panel, the $l v$-plot at $b=+0^{\circ} 30$ is shown 
and another one which is in observation since 1986 with the $7 \mathrm{~m}$ Bell-Labs-Antenna (Uchida et al. 1997). In addition, Jackson et al. (1996) have very recently announced a ${ }^{12} \mathrm{CO}(1-0)$ survey obtained with QUARRY at the Five College Radio Astronomy Observatory (FCRAO).

\subsection{Observations and data reduction}

For ${ }^{12} \mathrm{CO}$, the AOS frequency resolution per channel of $1.07339 \mathrm{MHz}$ corresponds to $2.79 \mathrm{~km} \mathrm{~s}^{-1}$ which resulted in a total velocity bandwidth of $715 \mathrm{~km} \mathrm{~s}^{-1}$. The ${ }^{12} \mathrm{CO}$ data were taken in March 1994 and August 1994. The observations were done in the upper sideband of the receiver with an image frequency of $112.506104 \mathrm{GHz}$. The observing procedure was the same as for the $\mathrm{C}^{18} \mathrm{O}(1-0)$ observations (see Appendix A).

To make comparisons easier, we made the ${ }^{12} \mathrm{CO}(1-0)$ measurements with the same spacing and coordinate alignment as the $\mathrm{C}^{18} \mathrm{O}$ survey. This has the disadvantage that the ${ }^{12} \mathrm{CO}$ data are slightly less than half sampled because the FWHM beam width of the telescope is 8.8 at the line frequency of the ${ }^{12} \mathrm{CO}(1-0)$ transition, but with this setup point by point comparison becomes possible. Our ${ }^{12} \mathrm{CO}$ map covers the range from $l=3.6$ to -0.9 and from $b=-0.3$ to $+0 \circ 3$. From $l=3.0$ to 3.6 the ${ }^{12} \mathrm{CO}$ observations could be extended to $b=+0.75$ which includes most of Clump 2.

The positions of the grid were typically observed once, with a single 10-minute-scan. In the rare case of bad weather conditions a position was measured twice, so that an $\mathrm{rms}$ noise temperature of about 0.15 to $0.25 \mathrm{~K}$ was achieved. The resulting signal-to-noise ratio of the ${ }^{12} \mathrm{CO}$ data is much better than that of the $\mathrm{C}^{18} \mathrm{O}$ data because the ${ }^{12} \mathrm{CO}$ emission is easily forty times stronger than the $\mathrm{C}^{18} \mathrm{O}$ emission. Thus, this rms is more than sufficient for a reliable comparison. The ofF positions used were the same as for the $\mathrm{C}^{18} \mathrm{O}$ observations (see Appendix $\mathrm{B}$ ).

The data reduction was done in the same way as for the $\mathrm{C}^{18} \mathrm{O}$ data. The baselines subtracted were typically of the order of 3 . Higher order baselines were not necessary, since due to the more intense line emission in ${ }^{12} \mathrm{CO}$, the long period sinusoidal waves (from reflections in the telescope) did not affect the spectra as much as in the case of $\mathrm{C}^{18} \mathrm{O}$.

As in $\mathrm{C}^{18} \mathrm{O}$, the calibration stability was monitored by taking a 10-minute-spectrum toward the position $l=$ $0.625, b=0.0$ (Sgr B2) once or twice per day. It was found to be as stable as for $\mathrm{C}^{18} \mathrm{O}$.

Also as in the case of $\mathrm{C}^{18} \mathrm{O}$, the scaling factor of the AOS calibration has to be determined. Again, we chose to compare the ${ }^{12} \mathrm{CO}(1-0)$ emission of Sgr B2 observed with the AOS at the SMWT to observations of the same source with the $0.5 \mathrm{MHz}$-resolution-filterbank at the $1.2 \mathrm{~m}$ NMWT (compare Sect. 3.4). To obtain sufficient bandwidth, it was necessary to merge sets of spectra centered on $-200,-100,0,100$, and $200 \mathrm{~km} \mathrm{~s}^{-1}$ because at ${ }^{12} \mathrm{CO}(1-0)$ the bandwidth of the 256-channel-filterbank of $128 \mathrm{MHz}$ corresponding to only $332.9 \mathrm{~km} \mathrm{~s}^{-1}$ was not sufficient. Then, the peak intensity of the SMWT and the NMWT were fitted.

With these fits, the "efficiency" of the AOS calibration at the ${ }^{12} \mathrm{CO}(1-0)$ line emission, $\eta_{\mathrm{AOS}-12} \mathrm{CO}$, is:

$\eta_{\mathrm{AOS}-{ }^{12} \mathrm{CO}}=1.536 \pm 0.076$.

Using this result, the following conversions for $T_{\mathrm{A}}^{*}$ and $T_{\mathrm{MB}}$ can be established:

$$
\begin{aligned}
T_{\mathrm{A}}^{*} & =\frac{T_{\mathrm{A}-\mathrm{AOS}}^{*}}{\eta_{\mathrm{AOS}-12 \mathrm{CO}}}=\frac{T_{\mathrm{A}-\mathrm{AOS}}^{*}}{1.536} \\
T_{\mathrm{MB}} & =\frac{T_{\mathrm{A}}^{*}}{\eta_{\mathrm{mf}}}=\frac{T_{\mathrm{A}-\mathrm{AOS}}^{*}}{\eta_{\mathrm{mf}} \eta_{\mathrm{AOS}-12} \mathrm{CO}}=\frac{T_{\mathrm{A}-\mathrm{AOS}}^{*}}{1.260} .
\end{aligned}
$$

\subsection{Results based on the ${ }^{12} C O(1-0)$ data}

In this section, we present the results based on the ${ }^{12} \mathrm{CO}(1-0)$ Galactic center data. The intensities of the data are scaled to $T_{\mathrm{MB}}$, following Eq. (9). In Fig. 14, the full set of survey spectra on the same grid used for $\mathrm{C}^{18} \mathrm{O}$ is given. The mean $\mathrm{rms}$ per channel of all 174 spectra is $166.6 \pm 67.7 \mathrm{mK}$.

\subsubsection{The integrated intensity of ${ }^{12} \mathrm{CO}(1-0)$}

The total integrated emission of ${ }^{12} \mathrm{CO}(1-0)$ is given in Fig. 15 as a contour map. This covers the velocity range from -225.0 to $+275.0 \mathrm{~km} \mathrm{~s}^{-1}$, i.e. the complete emission range of the Galactic center region in the observed area, as can be seen from the $l v$-plot in Fig. 17. The plot was produced in the same way as Fig. 5.

As in $\mathrm{C}^{18} \mathrm{O}$, one finds a coincidence of the main emission regions with the major continuum sources Sgr A (with an extension to Sgr B1) and Sgr B2, Sgr C and Sgr D. The emission near Sgr A and Sgr B1 appears as a ridge from $l=-0.02$ to +0.2 at $b=-0^{\circ} .03$. At the position of Sgr B2, ${ }^{12} \mathrm{CO}$ peaks at $(l=+0.72, b=-0.04)$. Near Sgr C, ${ }^{12} \mathrm{CO}$ peaks at $(l=-0.53, b=-0.12)$, and near Sgr D at $(l=+1.3, b=-0.1)$ with an extension to $(l=+1.05$, $b=-0.15)$. In addition, Clump 2 appears as a strong and widespread emission feature, peaking at $(l=+3$. 15 , $b=+0.3)$. A further comparatively weak maximum is at $(l=+1.95, b=-0.15)$. The global differences to the $\mathrm{C}^{18} \mathrm{O}$ data are:

(1) The ${ }^{12} \mathrm{CO}$ emission at Sgr B2 is not as strikingly strong compared to the other emission maxima.

(2) The ${ }^{12} \mathrm{CO}$ emission is much more widespread than the $\mathrm{C}^{18} \mathrm{O}$ emission. In fact, ${ }^{12} \mathrm{CO}$ emission is visible over all the map. However, the smaller latitude extent of the $\mathrm{C}^{18} \mathrm{O}$ emission might be influenced by the detection limit of the rather weak $\mathrm{C}^{18} \mathrm{O}(1-0)$ line.

(3) The ${ }^{12} \mathrm{CO}$ emission from Clump 2 is much stronger compared to the other features than in $\mathrm{C}^{18} \mathrm{O}$.

As in $\mathrm{C}^{18} \mathrm{O}$, the continuum sources G 0.50 .0 and G $0.9+$ 0.1 (Downes \& Maxwell 1966) do not have counterparts in ${ }^{12} \mathrm{CO}$. 


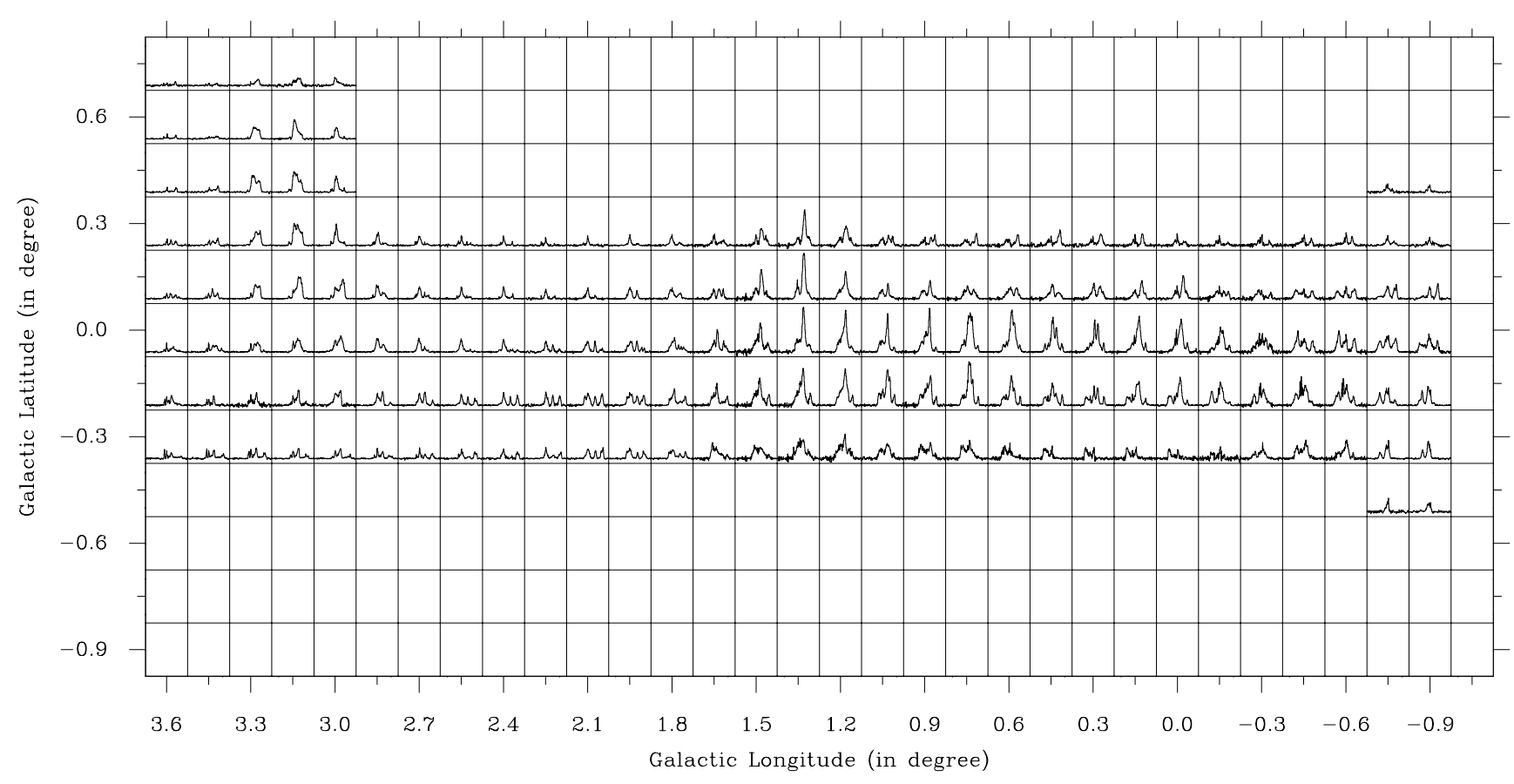

Fig. 14. The full set of spectra of our ${ }^{12} \mathrm{CO}(1-0)$ Galactic Center Survey. The $x$-axis of the spectra is $v_{\mathrm{LSR}}$ and ranges from -357.3 to $+357.3 \mathrm{~km} \mathrm{~s}^{-1}$, the $\mathrm{y}$-axis is $T_{\mathrm{MB}}$ and ranges from -1.5 to $15.5 \mathrm{~K}$. The longitude range of the plot is from $l=3.6$ to -1.05 and the latitude range is from $b=-0.9$ to +0.75

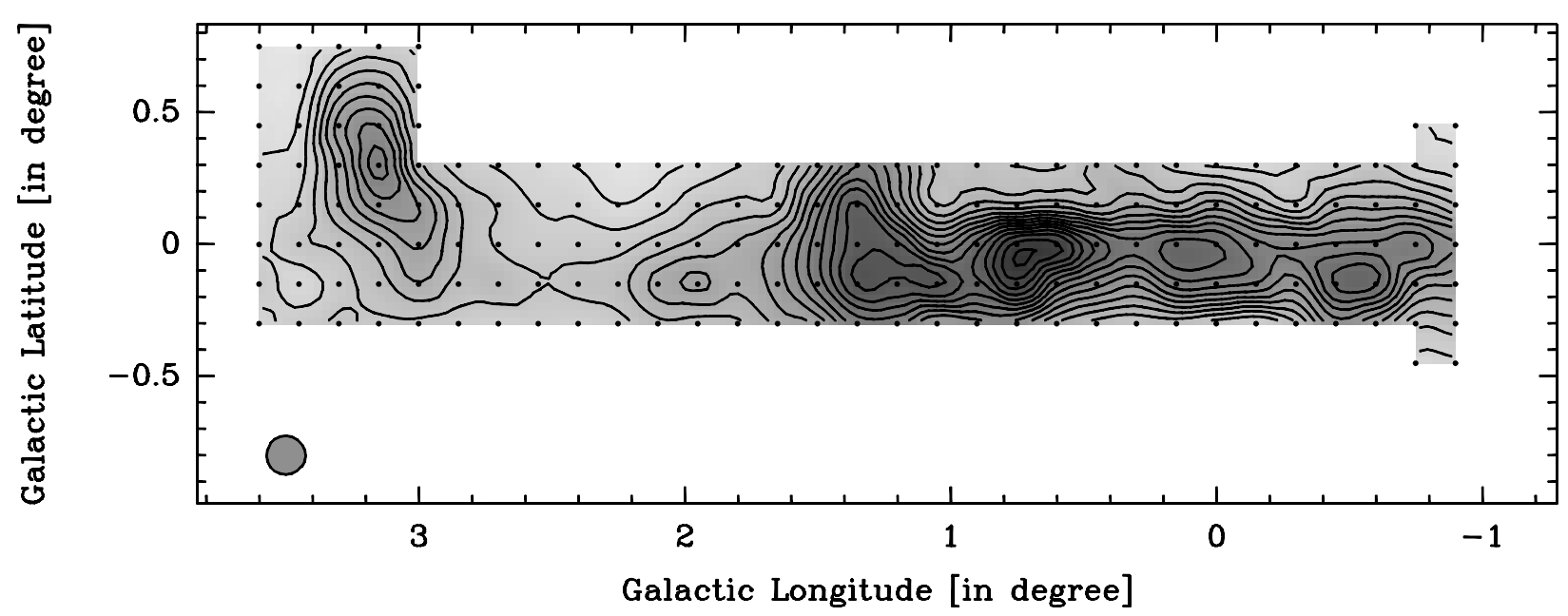

Fig. 15. The integrated intensity of the Galactic center region in ${ }^{12} \mathrm{CO}(1-0)$. The velocity the intensity is integrated over ranges from -225.0 to $+275.0 \mathrm{~km} \mathrm{~s}^{-1}$. The solid contour levels range from 150.0 to 1650.0 in steps of $100.0 \mathrm{Kkm} \mathrm{s}$. The minimum in the contour map is $51.4 \mathrm{~K} \mathrm{~km} \mathrm{~s}^{-1}$ which is well above the $3 \sigma$-value of $18.7 \mathrm{~K} \mathrm{~km} \mathrm{~s}^{-1}$. The circle in the lower left corner of the plot represents the beam size of 8.'8

Channel maps of $50 \mathrm{~km} \mathrm{~s}^{-1}$ width of ${ }^{12} \mathrm{CO}(1-0)$ are given in Fig. 16. The plots are produced in close analogy to Fig. 6. In the following description we mention only the major features. A more detailed discussion of the channel maps of the ${ }^{12} \mathrm{CO}$ data is given by Dahmen (1995). 

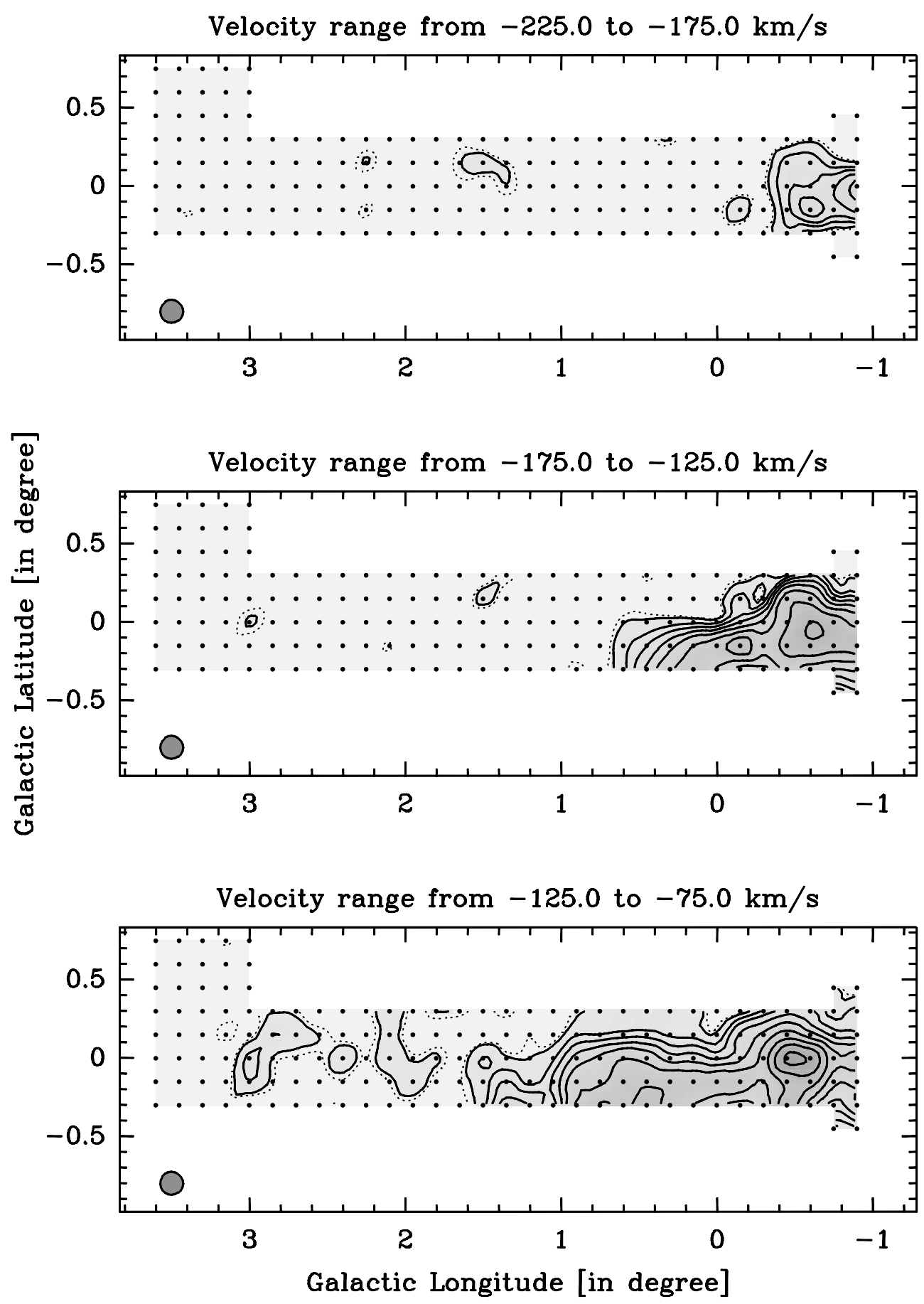

Fig. 16. a) The integrated intensity of the Galactic center region in ${ }^{12} \mathrm{CO}(1-0)$ in velocity intervals of $50 \mathrm{~km} \mathrm{~s}{ }^{-1}$ width. The solid contour levels are at 6.0 (which is the $3 \sigma$-level), 15.0, 25.0, 40.0, 60.0, 90.0, 130.0, 180.0, 240.0, 310.0, 390.0, 480.0, 580.0, and $690.0 \mathrm{~K} \mathrm{~km} \mathrm{~s}^{-1}$. The dashed contour is at $4.0 \mathrm{~K} \mathrm{~km} \mathrm{~s}^{-1}$ which is the $2 \sigma$-value. The circle in the lower left corner of the plots indicates the beam size of 8.8 . a) At the top the integrated intensity in the velocity range from -225.0 to $-175.0 \mathrm{~km} \mathrm{~s}{ }^{-1}$ is plotted, in the middle panel the velocity ranges from -175.0 to $-125.0 \mathrm{~km} \mathrm{~s}^{-1}$, and at the bottom the velocity range from -125 to $-75.0 \mathrm{~km} \mathrm{~s}^{-1}$ is shown 

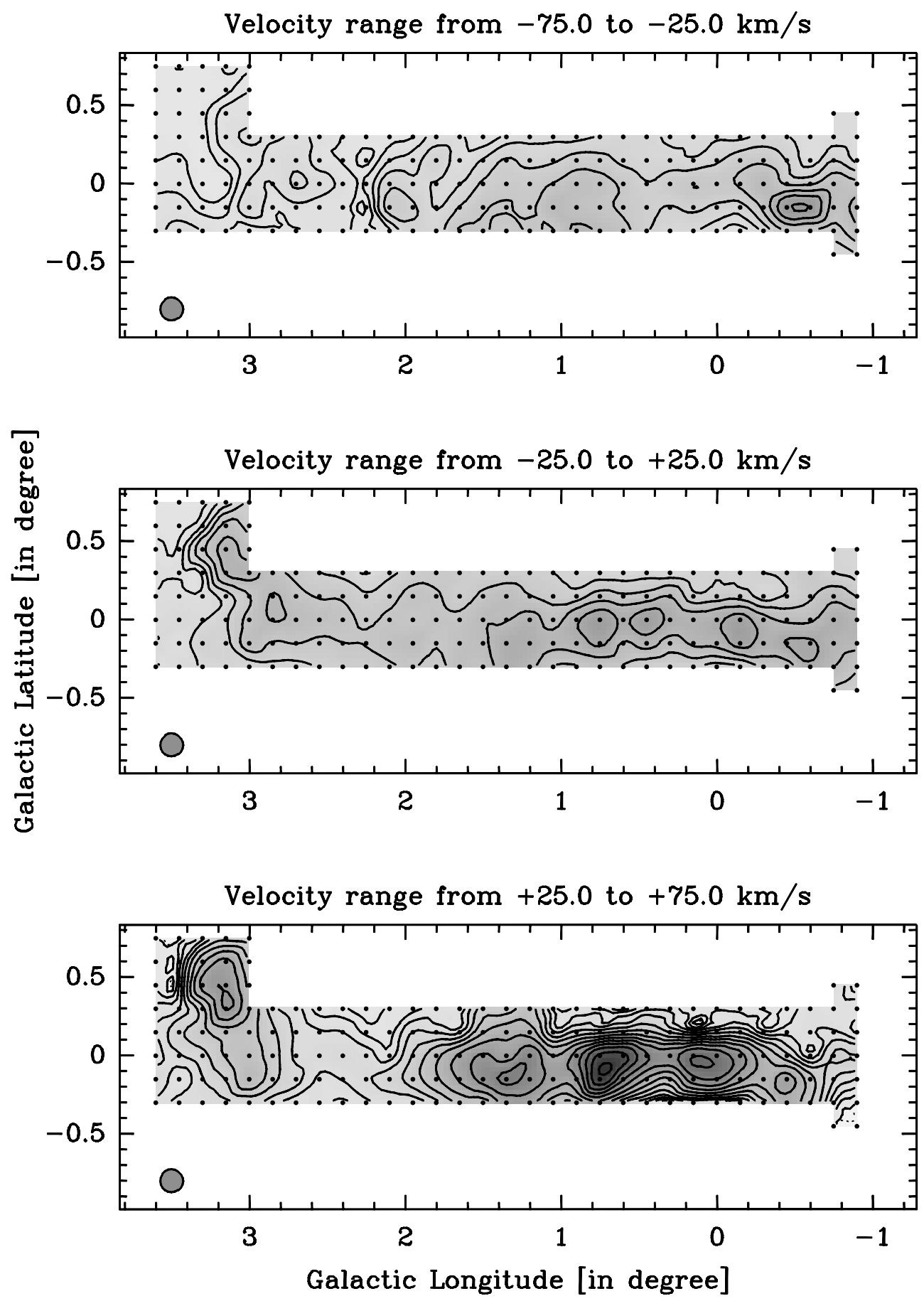

Fig. 16. b) At the top the integrated intensity of the velocity range from -75.0 to $-25 \mathrm{~km} \mathrm{~s}^{-1}$ is plotted, in the middle the velocity ranges from -25 to $+25 \mathrm{~km} \mathrm{~s}^{-1}$, and at the bottom the velocity range from +25 to $+75.0 \mathrm{~km} \mathrm{~s}^{-1}$ is displayed

At $-200 \mathrm{~km} \mathrm{~s}^{-1},{ }^{12} \mathrm{CO}$ appears at the negative longitude edge of the map. A maximum beyond $l=-0.9$, the border of the map, is indicated. In addition, a weak max- imum appears near $\operatorname{Sgr} C$ at $(l=-0.6, b=-0.13)$. At positive longitudes no ${ }^{12} \mathrm{CO}$ emission is visible. 

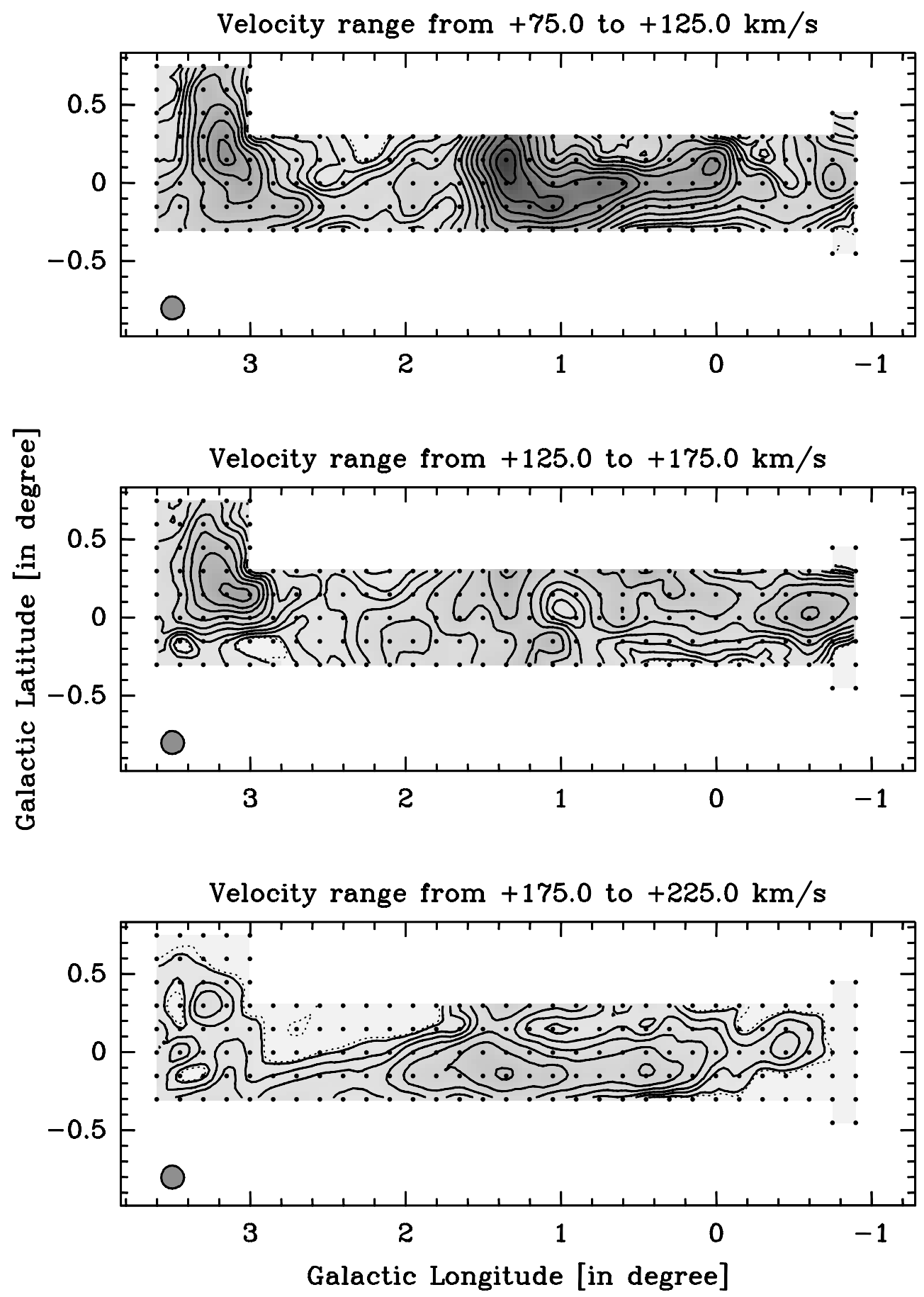

Fig. 16. c) At the top the integrated intensity of the velocity range from +75.0 to $+125 \mathrm{~km} \mathrm{~s}^{-1}$ is plotted, in the middle the velocity ranges from +125 to $+175 \mathrm{~km} \mathrm{~s}^{-1}$, and at the bottom the velocity range from +175 to $+225.0 \mathrm{~km} \mathrm{~s}^{-1}$ is displayed

At $-150 \mathrm{~km} \mathrm{~s}^{-1}$, the ${ }^{12} \mathrm{CO}$ emission has expanded toward $l=+0$. 7 . The main maximum appears near Sgr C at $(l=-0.65, b=-0.05)$.
At $-100 \mathrm{~km} \mathrm{~s}^{-1}$, continous ${ }^{12} \mathrm{CO}$ emission is extended toward higher longitudes up to $l=+1.6$. Beyond this (between $l=+1.8$ and +3 . 1 ) some isolated weak emission 

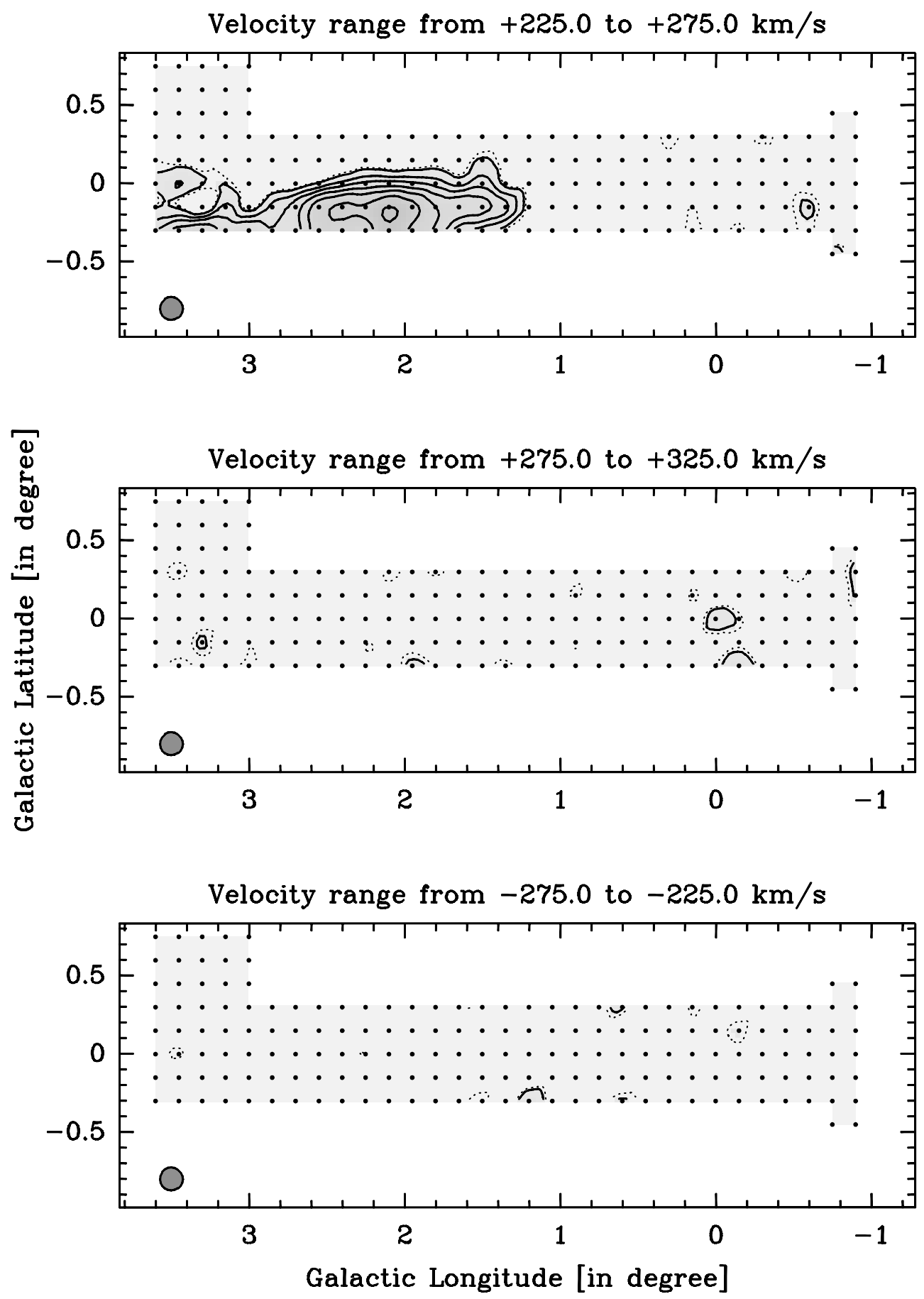

Fig. 16. d) At the top the integrated intensity of the velocity range from +225.0 to $+275 \mathrm{~km} \mathrm{~s}^{-1}$ is plotted, in the middle the velocity ranges from +275 to $+325 \mathrm{~km} \mathrm{~s}^{-1}$, and at the bottom the velocity range from -275 to $-225.0 \mathrm{~km} \mathrm{~s}^{-1}$ is displayed

areas are visible. The main peak appears near $\mathrm{Sgr} \mathrm{C}$ at $(l=$ $-0.5, b=0.0)$. A rather strong ridge starts at this peak and extends along the $b=-0.15$-line to about $l=+0.7$.
At $-50 \mathrm{~km} \mathrm{~s}^{-1}$, the complete map shows ${ }^{12} \mathrm{CO}$ emission at a significant level, associated with the 3-kpcarm gas. In general, the emission is widespread and 
comparatively weak with hardly any clear emission peaks. The ridge of maximum emission does not clearly follow the Galactic plane. The emission seems structureless, compared to the corresponding $\mathrm{C}^{18} \mathrm{O}$ emission. The only distinct peak appears at $(l=-0.52, b=-0.15)$ near Sgr C.

At $0 \mathrm{~km} \mathrm{~s}^{-1}$, the Galactic center ${ }^{12} \mathrm{CO}$ emission is superposed on the emission of the local gas. However, the local component does not contribute much to the integrated intensity because of its small linewidth. Thus, the integrated intensity is dominated by Galactic center gas. As at $-50 \mathrm{~km} \mathrm{~s}^{-1}$, the ${ }^{12} \mathrm{CO}$ emission shows little structure and fills the entire map. It is most widespread and uniformly distributed at this velocity. However, the ridge of emission follows the Galactic plane from the negative longitude edge of the map up to Clump 2 where it turns toward positive latitudes and ends at about $(l=+3.15, b=+0.5)$. Weak maxima appear near Sgr A $(l=-0.15, b=-0.8)$, Sgr B2 $(l=+0.75, b=-0.05)$, Sgr C $(l=-0.55$, $b=-0.05)$, and G $0.50 .0(l=+0.45, b=-0.02)$. This is the only correspondence of a ${ }^{12} \mathrm{CO}$ emission maximum with G 0.5 0.0. Clump 2 shows intense emission peaking at $(l=+3.1, b=+0.35)$. A minimum in the ridge of emission appears at about $l=+2.3$, but even there the emission remains strong. Thus, there is a rather strong connection in the emission of ${ }^{12} \mathrm{CO}$ between the inner Galactic center region and Clump 2.

At $+50 \mathrm{~km} \mathrm{~s}^{-1}$, there is much more structure in the ${ }^{12} \mathrm{CO}$ emission than at $0 \mathrm{~km} \mathrm{~s}^{-1}$ and $-50 \mathrm{~km} \mathrm{~s}^{-1}$. The Galactic center region appears as a strong ridge with maxima between Sgr A and Sgr B1 at $(l=+0.1, b=-0.05)$, near Sgr B2 at $(l=+0.72, b=-0.1), \operatorname{Sgr} \mathrm{C}$ at $(l=-0.58$, $b=-0.17)$, and $\operatorname{Sgr} \mathrm{D}$ at $(l=+1.3, b=-0.12)$. The emission from Clump 2 is stronger at $+50 \mathrm{~km} \mathrm{~s}^{-1}$ than at $0 \mathrm{~km} \mathrm{~s}^{-1}$ and peaks at $(l=+3.15, b=+0.35)$. The emission bridge between Clump 2 and the inner Galactic center region is weaker than at $0 \mathrm{~km} \mathrm{~s}^{-1}$ and has a minimum at about $l=+2.5$. In general, the emission is almost as widespread as at $0 \mathrm{~km} \mathrm{~s}^{-1}$ and $-50 \mathrm{~km} \mathrm{~s}^{-1}$. However, the contrast between the maxima and the minima is much higher. Sgr B2 is the strongest maximum of all, but not as prominent as in $\mathrm{C}^{18} \mathrm{O}$.

At $+100 \mathrm{~km} \mathrm{~s}^{-1}$, $\mathrm{Sgr} \mathrm{D}$ is, as in $\mathrm{C}^{18} \mathrm{O}$, the dominant ${ }^{12} \mathrm{CO}$ emission feature (the $l=1.5$-complex of Bally et al. 1988). However, it peaks at a distinctly different position than in $\mathrm{C}^{18} \mathrm{O}$, namely at $(l=+1.35$, $b=+0$. 15$)$. The ridge of stronger emission starts there and continues toward about $(l=+1.3, b=-0.1)$, turns toward lower longitudes and remains rather strong toward $(l=+0.6, b=0$. 0$)$. Then it follows the $b=0$. 0 -line toward $l=+0.15$ where it turns into the weak maximum near Sgr A at $(l=+0.12, b=+0.02)$ which is the end of the ridge. This ridge is also visible in $\mathrm{C}^{18} \mathrm{O}$ where it has nearly the same extent. In addition, Clump 2 is also rather strong at this velocity, but peaks at a somewhat different position, namely at $(l=+3.15, b=+0.2)$. As at $+50 \mathrm{~km} \mathrm{~s}^{-1}$, there exists a bridge of emission between Clump 2 and the inner Galactic center region.

At $+150 \mathrm{~km} \mathrm{~s}^{-1}$, the ${ }^{12} \mathrm{CO}$ emission is still widespread and covers nearly the complete map whereas the $\mathrm{C}^{18} \mathrm{O}$ emission has completely disappeared. However, the shape of the emission has changed greatly compared to lower velocities. In the inner Galactic center region the emission peaks at $(l=-0.62, b=+0.05)$, near Sgr C. There is a clear minimum of emission at $(l=+1.0, b=+0.05)$. Clump 2 has a strength similar to that of Sgr C and peaks at $(l=+3.05, b=+0.15)$. The emission bridge between Clump 2 and the inner Galactic center region is also present but weaker than at lower velocities.

At $+200 \mathrm{~km} \mathrm{~s}^{-1}$, the intensity of the ${ }^{12} \mathrm{CO}$ emission starts to decline at negative longitudes and positive latitudes. In general, the emission is distinctly weaker than at lower velocities. The main maximum is near $\operatorname{Sgr} \mathrm{D}$ at $(l=+1.35, b=-0.15)$. At longitudes higher than $l=+2.0$ an emission ridge at negative latitudes arises. Clump 2 shows widespread but weak emission without any clear peak.

At $+250 \mathrm{~km} \mathrm{~s}^{-1}$, the ${ }^{12} \mathrm{CO}$ emission from the inner Galactic center region and in Clump 2 has completely disappeared. But there is emission at higher positive longitudes and negative latitudes which first appears at $+200 \mathrm{~km} \mathrm{~s}^{-1}$. It covers the range from $l=+1.2$ to +3.6 and perhaps to higher longitudes beyond the edge of the map and from $b=+0.1$ to -0.3 , probably continuing toward lower latitudes.

The middle and the lower plot of Fig. $16 \mathrm{~d}$ show the channel maps at $+300 \mathrm{~km} \mathrm{~s}^{-1}$ and at $-250 \mathrm{kms}^{-1}$. These confirm that outside the range from $-225 \mathrm{~km} \mathrm{~s}^{-1}$ to $+275 \mathrm{~km} \mathrm{~s}^{-1}$ no ${ }^{12} \mathrm{CO}(1-0)$ emission is visible.

\subsubsection{Longitude-velocity plots}

In Fig. 17, we have plotted the intensity integrated over the latitude range from -0.30 to +0.30 , similar to Fig. 7 . In the following description we mention only the major features.

In the $l v$-space, the ${ }^{12} \mathrm{CO}(1-0)$ emission appears as a broad strip with constant velocity width of about $375 \mathrm{~km} \mathrm{~s}^{-1}$, only the center of which shifts with longitude. At the lower longitude edge of the map $(l=-0.9)$ this center velocity is $\approx-30 \mathrm{~km} \mathrm{~s}^{-1}$, it shifts to $\approx+90 \mathrm{~km} \mathrm{~s}^{-1}$ at $l \approx+2.0$, and remains constant from there up to the upper longitude edge of the map at $l=+3$. 6 . In general, the ${ }^{12} \mathrm{CO}$ emission is much more widespread and homogeneous than the $\mathrm{C}^{18} \mathrm{O}$ emission. The strongest feature is not Sgr B2, as in $\mathrm{C}^{18} \mathrm{O}$, but the 1.5-complex near Sgr D peaking at $\left(l \approx+1.3, v \approx+90 \mathrm{~km} \mathrm{~s}^{-1}\right)$ which has the same position as the corresponding $\mathrm{C}^{18} \mathrm{O}$ peak. In addition to this peak, only two maxima are clearly present: Sgr B2 at $\left(l \approx+0.7, v \approx+50 \mathrm{~km} \mathrm{~s}^{-1}\right)$ and Clump 2 at $\left(l \approx+3.15, v \approx+95 \mathrm{~km} \mathrm{~s}^{-1}\right)$. The emission of gas near $0 \mathrm{~km} \mathrm{~s}^{-1}$ with respect to the LSR frame appears at about 


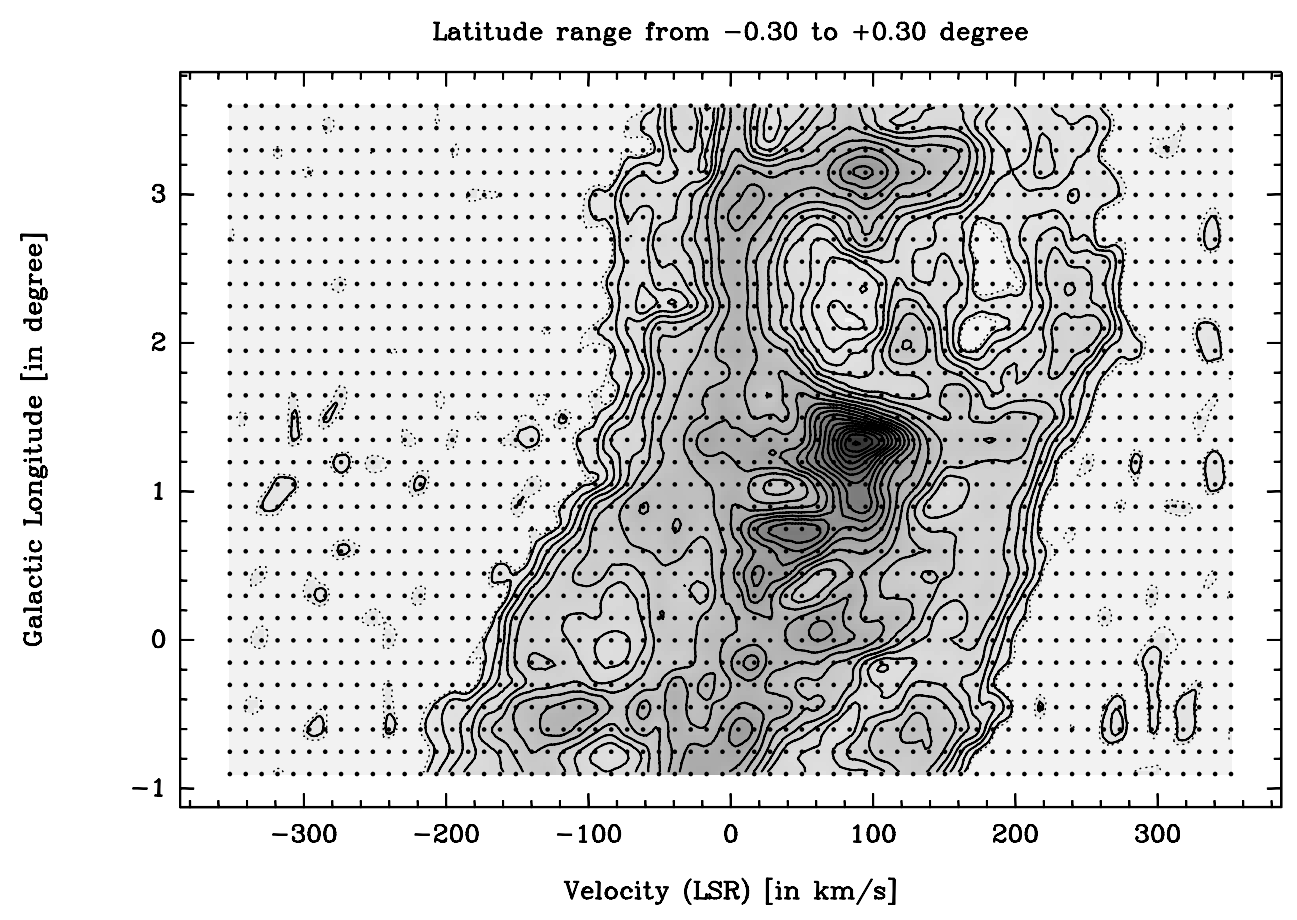

Fig. 17. Longitude-velocity plot showing the velocity distribution of the ${ }^{12} \mathrm{CO}(1-0)$ emission in the latitude range from -0.30 to $+0^{\circ} 30$. The solid contour levels are at $0.084 \mathrm{~K}$ arcdeg (which is the $3 \sigma$-level), $0.2,0.35,0.55,0.8,1.1 \mathrm{~K}$ arcdeg, and then range from 1.5 to 8.0 in steps of $0.5 \mathrm{~K}$ arcdeg. The dashed contour is at $0.056 \mathrm{~K}$ arcdeg which is the $2 \sigma$-value

$+5 \mathrm{~km} \mathrm{~s}^{-1}$ for longitudes larger than $+2^{\circ}$. At lower longitudes a ridge of this gas is not distinguishable from the overall emission but strong emission is always present at this velocity. The 3 -kpc-arm, at about $-50 \mathrm{~km} \mathrm{~s}^{-1}$, is visible in the complete longitude range of the map. However, it is more patchy than the gas near $0 \mathrm{~km} \mathrm{~s}^{-1}$. The diagonal chain of emission centers which is very prominent in $\mathrm{C}^{18} \mathrm{O}$ is represented in ${ }^{12} \mathrm{CO}$ by the prominent peaks Sgr B2 and Sgr D. Sgr C and Sgr A are seen only as weak maxima at $\left(l \approx-0.5, v \approx-40 \mathrm{~km} \mathrm{~s}^{-1}\right)$ and $\left(l \approx-0.15, v \approx-10 \mathrm{~km} \mathrm{~s}^{-1}\right)$, respectively. On the other hand, the emission tail, which ranges from Sgr A to about $\left(l \approx+0.3, v \approx+80 \mathrm{~km} \mathrm{~s}^{-1}\right)$ in $\mathrm{C}^{18} \mathrm{O}$, is also rather prominent in ${ }^{12} \mathrm{CO}$ and seems to connect to the 1.5-complex and to the Expanding Molecular Ring (EMR, Scoville 1972; Kaifu et al. 1972) at about $+170 \mathrm{kms}^{-1}$. From the inner Galactic center region toward Clump 2, a connecting bridge of emission exists not only at $0 \mathrm{~km} \mathrm{~s}^{-1}$ but also at $+120 \mathrm{~km} \mathrm{~s}^{-1}$, connecting the maxima of the 1.5 -complex and of Clump 2. This $+120 \mathrm{~km} \mathrm{~s}^{-1}$-bridge has a maximum at $\left(l \approx+2.0, v \approx+120 \mathrm{~km} \mathrm{~s}^{-1}\right)$. More prominent minima of emission are visible at $\left(l \approx+0.35, v \approx+55 \mathrm{~km} \mathrm{~s}^{-1}\right)$, at $\left(l \approx+1.0, v \approx+30 \mathrm{~km} \mathrm{~s}^{-1}\right)$, in the range from $l=+2.0$ to 2.5 and $v=+60$ to $+100 \mathrm{~km} \mathrm{~s}^{-1}$, and in the range from $l=+2.0$ to 2.8 and $v=+165$ to $+195 \mathrm{~km} \mathrm{~s}^{-1}$.
A more detailed discussion of this latitude integrated plot of the ${ }^{12} \mathrm{CO}$ data can be found in Dahmen (1995). There, $l v$-plots for selected latitudes of the ${ }^{12} \mathrm{CO}$ data, analogous to Fig. 8, are also presented and discussed in detail.

\subsubsection{Latitude-velocity plots}

In Fig. 18, we show the intensity integrated over two longitude ranges, the inner Galactic center from -0.90 to +2.025 (a) and Clump 2 from +2.025 to +3.6 (b), in analogy to Fig. 9.

In general, the emission in $b v$-space has a limited amount of structure, compared to the $l v$-plot. However, some interesting features can be recognized, in particular in comparison to the $\mathrm{C}^{18} \mathrm{O}$ data.

In contrast to $\mathrm{C}^{18} \mathrm{O}$, the ${ }^{12} \mathrm{CO}$ emission in plot "a)" appears as a bulge-like plateau in the range from $b=-0.2$ to +0.05 and $v=+15$ to $+100 \mathrm{~km} \mathrm{~s}^{-1}$. The maximum of emission appears at $\left(b=+0.05, v=+85 \mathrm{~km} \mathrm{~s}^{-1}\right)$, and the $v$-extent of the emission is from -210 to $+280 \mathrm{~km} \mathrm{~s}^{-1}$. Rather strong extensions of the emission are visible along the $v=0 \mathrm{~km} \mathrm{~s}^{-1}$ line, toward $\operatorname{Sgr} \mathrm{C}(b=-0.15, v=$ $\left.-40 \mathrm{~km} \mathrm{~s}^{-1}\right)$ and toward the 1.5-complex $(b=+0.2, v=$ $\left.+110 \mathrm{~km} \mathrm{~s}^{-1}\right)$. 


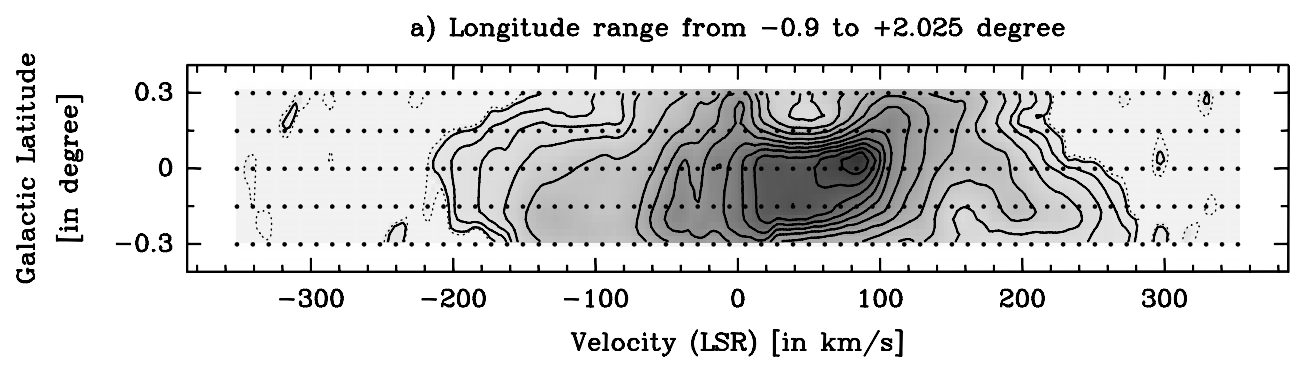

b) Longitude range from +2.025 to +3.6 degree

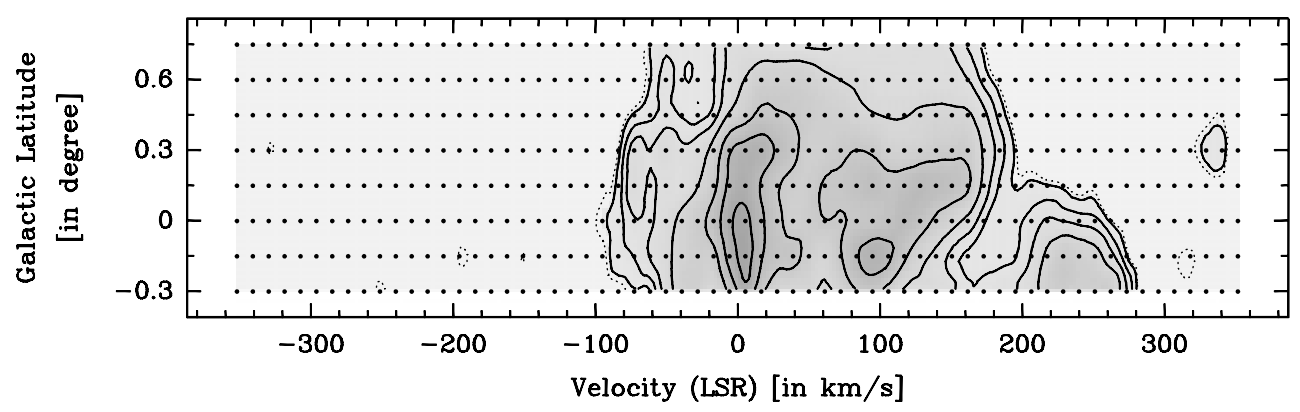

Fig. 18. Latitude-velocity plots showing the velocity distribution of the ${ }^{12} \mathrm{CO}(1-0)$ emission. a) The longitude range from -0.90 to +2.025 . The solid contour levels are at 0.18 (which is the $3 \sigma$-level), $0.45,0.9$, and then range from 1.8 to 18.0 in steps of $1.8 \mathrm{~K}$ arcdeg. The dashed contour is at $0.12 \mathrm{~K}$ arcdeg which is the $2 \sigma$-value. $\mathbf{b}$ ) The longitude range from +2.025 to +3.60 . The solid contour levels are at 0.12 (which is the $3 \sigma$-level), $0.3,0.6$, and then range from 1.2 to 4.8 in steps of $1.2 \mathrm{~K}$ arcdeg. The dashed contour is at $0.08 \mathrm{~K}$ arcdeg which is the $2 \sigma$-value

Plot "b)" in Fig. 18 shows 3 emission features at about $+5 \mathrm{~km} \mathrm{~s}^{-1},+95 \mathrm{~km} \mathrm{~s}^{-1}$ and $+240 \mathrm{~km} \mathrm{~s}^{-1}$. The first two components are also (although not as widespread) visible in $\mathrm{C}^{18} \mathrm{O}$. These represent Clump 2 with its rather large extension toward positive $b$-values. The high velocity component which is restricted to negative latitudes is not visible in $\mathrm{C}^{18} \mathrm{O}$ and represents the elongated ridge visible in the channel maps of $+200 \mathrm{~km} \mathrm{~s}^{-1}$ and in particular of $+250 \mathrm{~km} \mathrm{~s}^{-1}$ (Fig. 16).

\subsection{Comparison with other ${ }^{12} \mathrm{CO}(1-0)$ measurements}

If one compares the ${ }^{12} \mathrm{CO}$ data presented here with the more than 20 years old data of Bania $(1977,1980,1986)$, one first has to take into account that the data of Bania are by a factor of 10 undersampled latitude cuts at $b=-20^{\prime}$, $-10^{\prime}, 0^{\prime},+10^{\prime}$, and $+20^{\prime}$. Thus, these have to be compared with latitude cuts of our ${ }^{12} \mathrm{CO}$ data. Doing this (see Dahmen 1995), it is obvious that Bania's survey has a distinctly lower sensitivity than the observations presented here, even though our data are just 10-minutescans. However, with these restrictions in mind, both data sets show the same features. A comparison of the absolute intensities is not possible because Bania gives only $T_{\mathrm{A}}^{*}$ for his data. However, as already noted, there exist more recent and more complete data sets, one observed in 1984 with the 1.2 m SMWT (Bitran 1987; Bitran et al. 1997), another one which is in observation since 1986 with the $7 \mathrm{~m}$ Bell-Labs-Antenna (Uchida et al. 1997), and one observed in 1993 and 1994 with the FCRAO QUARRY (Jackson et al. 1996). The data of Bitran (1987) and Uchida et al. (1997) are available to us in digital format so that a more detailed comparison was possible.

\subsubsection{Earlier measurements with the $1.2 \mathrm{~m}$ SMWT}

Previous to the ${ }^{12} \mathrm{CO}(1-0)$ observations presented here the Galactic center region was surveyed by Bitran in the time from February to November 1984 and published in his Ph.D. thesis (Bitran 1987; see also Bitran et al. 1997). This survey covers a much larger area, is slightly better sampled $(7 ! 5)$ and has twice the sensitivity of our ${ }^{12} \mathrm{CO}$ observations. We performed a detailed comparison (see Dahmen 1995) between our data and Bitran's to make sure that both data sets are compatible for further analysis.

A comparison of the ${ }^{12} \mathrm{CO}$ Sgr B2 spectrum obtained at the $1.2 \mathrm{~m}$ NMWT in February 1995 with Bitran's respective spectrum shows that over a time scale of 10 years 
and for two different telescopes with different receivers (even though the telescopes are of the same design) the calibration agrees to within $10 \%$ which we consider to be excellent. In addition, the shapes of the observed spectra agree very well.

Contour maps of the integrated intensity of our ${ }^{12} \mathrm{CO}$ data (Fig. 15) and Bitran's survey are in excellent agreement. Both the shape and the location of the visible features are identical which indicates that the system and the pointing of the telescope were very stable over a time scale of 10 years.

Channel maps obtained from Bitran's ${ }^{12} \mathrm{CO}$ data are also in very close agreement with those obtained from our data, as are $l v$-plots. The maxima of the contour plots from Bitran's data, converted to $T_{\mathrm{MB}}$, tend to be $\sim 15 \%$ higher than the corresponding values of our data. Thus, the scaling of the integrated intensity is different from the scaling of a single spectrum by $5 \%$. It is, however, not clear if this difference is in fact significant.

Summarizing, our ${ }^{12} \mathrm{CO}$ data are in excellent agreement with the data of Bitran (1987). The system and the pointing of the telescope are found to be stable over a time scale of 10 years. Only the calibration deviates by $10 \%$ to $15 \%$. However, on a time scale of 10 years and taking into account that the calibration accuracy of the $1.2 \mathrm{~m} \mathrm{NMWT}$ (which was the standard for the data presented here) has recently been improved, this deviation seems to be rather small.

6.3.2. Recent measurements with the $7 \mathrm{~m}$ Bell-LabsAntenna

A large ${ }^{12} \mathrm{CO}(1-0)$ survey of the Galactic center region is being performed with the $7 \mathrm{~m}$ Bell-Labs-Antenna with 1.5 spacing and $2.6 \mathrm{~km} \mathrm{~s}^{-1}$ velocity resolution (Uchida et al. 1997). The survey currently covers a region from $l=-2.25$ to +3.75 in longitude and from $b=-0.6$ to +0.6 in latitude.

Using COMB, the AT\&T Bell Laboratories data reduction package, the data cube was resampled to our resolution in $l, b$ and $v$. All work at the $7 \mathrm{~m}$ telescope is done on a $T_{\mathrm{A}}^{*}$ scale that cannot be readily converted to $T_{\mathrm{MB}}$, preventing the comparison of the absolute calibration of the different data sets. However, the lineshapes of individual spectra toward prominent positions once again agree closely. Contour plots of the total integrated intensity, channel maps and $l v$-plots are in excellent agreement with our and with Bitran's data. A detailed discussion of this can be found in Dahmen (1995).

All three telescope systems have achieved essentially identical results in ${ }^{12} \mathrm{CO}$ when convolved to the same resolution, and we can thus be confident in the accuracy of calibration and pointing of the $\mathrm{C}^{18} \mathrm{O}$ data.

\section{Conclusions}

The large scale survey of the Galactic center region in the $\mathrm{C}^{18} \mathrm{O}(J=1 \rightarrow 0)$ transition line presented here reveals several interesting aspects of the molecular gas near the Galactic center.

(1) The spectra and maps of the $\mathrm{C}^{18} \mathrm{O}(1-0)$ survey demonstrate that there are great differences in the distribution of the optically thin $\mathrm{C}^{18} \mathrm{O}(1-0)$ emission and the ${ }^{12} \mathrm{CO}(1-0)$ emission, generally assumed to be optically thick.

(2) Compared to the ${ }^{12} \mathrm{CO}(1-0)$ emission, the $\mathrm{C}^{18} \mathrm{O}(1-0)$ emission shows much more contrast. This results, as will be invesigated in detail in Paper II, in line intensity ratios of ${ }^{12} \mathrm{CO}(1-0)$ to $\mathrm{C}^{18} \mathrm{O}(1-0)$ which are rather high and variable compared to ratios found in the Galactic disk.

(3) The occurence of molecular gas at velocities strictly forbidden by uniform circular motion is confirmed from $\mathrm{C}^{18} \mathrm{O}(1-0)$. Also confirmed is the displacement by several scale heights above (Sgr D, Clump 2) or below $(\mathrm{Sgr} \mathrm{C})$ the $b=0^{\circ}$-plane.

(4) The emission of the $\operatorname{HNCO}\left(5_{0,5}-4_{0,4}\right)$ is not only rather strong in the Galactic Center but also rather extended, though more confined than $\mathrm{C}^{18} \mathrm{O}$. In particular the feature HNCO-1.65-0.0 is of interest because it has no clear counterpart in $\mathrm{CO}$.

These properties of the molecular gas and especially the differences between the $\mathrm{C}^{18} \mathrm{O}(1-0)$ and the ${ }^{12} \mathrm{CO}(1-$ 0 ) emission will be discussed in Paper II (Dahmen et al. 1997). In addition, the physical and kinematic conditions of the Galactic center region will be discussed.

Acknowledgements. We acknowledge the financial support of a Bennigsen-Foerder research prize awarded to one of us (RM) by the Land Nordrhein-Westfalen, which was essential to carry out this work. Additional financial support for this project was awarded to two us (TLW and LB) by the Commission of the European Communities (contract CI1*-CT93-0332). LB and JM acknowledge support from FONDECYT Grant 1950627. We thank Prof. R. Wielebinski for the generous loan of the AOS, the staff of the Digital Laboratory of the MPIfR for the great support in maintaining the AOS, the people of the Departamento de Astronomía of the Universidad de Chile for the operation of the $1.2 \mathrm{~m}$ SMWT at CTIO, and the staff of the Cerro Tololo Interamerican Observatory (CTIO), in particular the engineers G. Arriagada and M. Lazo who were very helpful in solving technical problems. C. Henkel contributed great support during the preparation phase of this project. K.I. Uchida kindly gave us access to the Bell Labs ${ }^{12} \mathrm{CO}$ data before publication. Not at least, we very much thank all additional observers of this project who have all stayed on Cerro Tololo at least for two and mostly for three weeks, namely C. Lemme, D. Muders, H. Wiesemeyer, F. Wyrowski, T. Megeath, M. Petr, S. Richter, A. Grunert, S. Kohle, and D. Fischer. Finally, we thank the referee J. Bally for helpful comments. 


\section{Appendix}

\section{A. Observing procedure}

\section{A.1. Integration with weighted-OFFs position switching}

Because of the large velocity range covered by the Galactic Center emission, position switching was chosen as the observing mode. This observing mode produces flat baselines only if the atmospheric conditions for the ON and OFF positions are very similar, i.e., if their positions on the sky are not very different and the weather conditions are stable. Unfortunately, the Galactic Center region shows extremely extended emission, so that nearby emission-free positions are difficult to find, in particular with a $9^{\prime}$ telescope beam. Therefore, the OFF positions often had to be several degrees distant from the on position.

Flat baselines result if the difference power, DP, between the OFF and the ON positions is minimized. To achieve this, the following scheme is used (weighted-OFFs mode): The telescope control program picks two OFF positions from a given list of emission-free positions, one at higher and one at lower elevation than the oN position based on a weight assigned to each OFF position for its horizontal distance on the sky from the on position and for the predicted DP, from an atmospheric model, due to the distance in elevation. This elevation weighting takes into account the increment of air masses with decreasing elevation as well as the fact that, in worse weather conditions, there must be a smaller distance in elevation of appropriate OFF positions to the ON position. The weighting formula is as follows:

$W_{i}=\left(\frac{\Delta A z_{i} \cos \left(E l_{i}\right)}{\text { azweight } t_{1 \mathrm{Kdp}}}\right)^{2}+\left(T P_{\text {on }}-T P_{i}\right)^{2}$

where the second term is the predicted difference power, DP, squared. The term, azweight $t_{1 \mathrm{Kdp}}$, is the normalization factor for the distance in azimuth which indicates what distance in azimuth in degrees is weighted equally to a DP of $1 \mathrm{~K}$.

The choice of this normalization factor had to be empirical because distance in azimuth and difference power are very different quantities. In principle, azimuth changes should not matter, since these do not produce any systematic change in power. But atmospheric variations from position to position do occur. Also, as one switches further in azimuth, the time between ON and OFF increases, so that there are gain variations which increase with time. At the $1.2 \mathrm{~m}$ SMWT, 0.65 was determined to be the best choice for azweight $t_{1 \mathrm{Kdp}}$.

After weighting all the OFF positions for their current azimuth and elevation, the program chooses the best ofF position (lowest weight) as the first OFF (subscan A) and the best OFF position on the opposite side in elevation of the ON position as second OFF (subscan B). If the first OFF has an extremely low weighting value (nearly the same elevation as the ON position) only this OFF may be used for the complete scan. See Dame (1992) for further details on the weighted-OFFs mode.

The integration of the two subscans was done in 30second-cycles, the first half of which was spent on the OFF position. Note that the integration time of the scans is the sum of the integration time on source and on the ofF positions.

\section{A.2. Scan calibration}

\section{A.2.1. Obtaining $T_{\mathrm{A}}^{*}$}

Every subscan is started with a calibration lasting 5 seconds. The calibration procedure follows the standard chopper wheel method first described by Penzias \& Burrus (1973) and applied at many other millimeter telescopes. Following this method, the temperature in channel $i$ is calculated from:

$T_{i}=\frac{V_{i}^{\mathrm{ON}}-V_{i}^{\mathrm{OFF}}}{V_{i}^{\mathrm{CHOP}}-V_{i}^{\mathrm{SKY}}} \cdot T_{\mathrm{CAL}}$

where $V_{i}$ are the voltage outputs of each channel during the scan integration on source $\left(V_{i}^{\mathrm{ON}}\right)$, on the ofF position $\left(V_{i}^{\mathrm{OFF}}\right)$, during the calibration on the chopper wheel $\left(V_{i}^{\mathrm{CHOP}}\right)$, and on the sky $\left(V_{i}^{\mathrm{SKY}}\right)$, see Ulich \& Haas (1976), Downes (1989) for details. $T_{\mathrm{CAL}}$ is the elevation and weather dependent conversion factor from the measured voltage to the antenna temperature $T_{\mathrm{A}}^{*}$. This factor corrects for atmospheric attenuation in the signal and image sideband, and implicitly for resistive losses and rearward spillover and scattering (see Eq. (4) in Downes 1989 and Eq. (17) in Ulich \& Haas 1976).

Table 3. Opacities and temperatures of atmospheric oxygen on Cerro Tololo

\begin{tabular}{lll} 
& $\mathbf{1 2}^{\mathbf{1 2}}$ & $\mathbf{C}^{\mathbf{1 8}} \mathbf{O}$ \\
\hline$\tau_{\text {signal }}$ & 0.2054 & 0.0380 \\
$\tau_{\text {image }}$ & 0.0971 & 0.0650 \\
$T_{\text {signal }}$ & $255.0 \mathrm{~K}$ & $255.0 \mathrm{~K}$ \\
$T_{\text {image }}$ & $254.0 \mathrm{~K}$ & $254.0 \mathrm{~K}$
\end{tabular}

Generally, the calibration conversion factor, $T_{\mathrm{CAL}}$, is determined by carrying out an antenna tip, a procedure in which $T_{\mathrm{SKY}}$ is measured as a function of air mass using a two-layer atmospheric model, consisting of a timeconstant upper layer of $\mathrm{O}_{2}$ and a variable lower layer of $\mathrm{H}_{2} \mathrm{O}$, as described in Kutner (1978). The values for the opacity and temperature of the oxygen "layer" as used for the Cerro Tololo (altitude $2215 \mathrm{~m}$ ) are given in Table 3. The antenna tip has to be repeated periodically; every six hours was found to be adequate. The zenith opacity of 
water vapour was typically $\sim 0.1$. A more detailed description of this calibration method is given in Appendix $\mathrm{A}$ of Cohen et al. (1986).

\section{A.2.2. Obtaining $T_{\mathrm{MB}}$}

Because of the possibility of confusion caused by different notations found in the literature, we give a full account of the relevant relations.

The antenna temperature $T_{\mathrm{A}}^{*}$ is the natural result of chopper-wheel calibration. However, $T_{\mathrm{A}}^{*}$ is not appropriate for telescope and line independent comparisons because it is not corrected for all telescope losses (see, e.g., Downes 1989). $T_{\mathrm{A}}^{*}$ is the brightness temperature of an equivalent source which fills the entire $2 \pi$ steradians of the forward beam pattern; it can be thought of as a "forward-beam brightness temperature". Therefore, $T_{\mathrm{A}}^{*}$ still contains the forward beam pattern as a telescope dependent parameter. Besides the main beam (MB), this consists of (1) the spillover of feed power around the secondary, (2) the scattering from the aperture blockage caused by the secondary mirror and its support structure, and (3) the diffraction sidelobes through the finite aperture of the main mirror (Cohen et al. 1986). The appropriate parameter for comparison is the "main-beam brightness temperature", $T_{\mathrm{MB}}$, that is the brightness temperature of a source which just fills the main beam. $T_{\mathrm{MB}}$ is a property of the source itself; provided the source is resolved, different radio telescopes will have the same value.

In the notation of Downes (1989), the forward efficiency, $F_{\text {eff }}$, is defined as the factor which scales $T_{\mathrm{A}}^{\prime}$, the antenna temperature of an equivalent resistor outside the atmosphere (thus, the antenna temperature corrected for atmospheric losses), to $T_{\mathrm{A}}^{*}$ :

$T_{\mathrm{A}}^{\prime}=F_{\mathrm{eff}} T_{\mathrm{A}}^{*}$.

Following the notation of Kraus (1986), the forward efficiency is:

$F_{\mathrm{eff}}=\frac{T_{\mathrm{A}}^{\prime}}{T_{\mathrm{A}}^{*}}=k_{0} \frac{\Omega_{F}}{\Omega_{A}}$

where $k_{0}$ is the resistive loss factor of the telescope, $\Omega_{F}$ the forward-beam solid angle, $\Omega_{A}$ the antenna-beam solid angle. The two latter are defined as follows:

$$
\begin{aligned}
\Omega_{F} & =\iint_{\text {forward } 2 \pi} P_{n}(\vartheta, \varphi) \mathrm{d} \Omega \\
\Omega_{A} & =\iint_{4 \pi} P_{n}(\vartheta, \varphi) \mathrm{d} \Omega
\end{aligned}
$$

where $P_{n}(\vartheta, \varphi)$ is the antenna power pattern normalized to its maximum value as a function of angle. Thus, a beam solid angle is the angle inside of which a fictitious antenna must have a power pattern equal to the maximum value of the antenna in use and outside which an antenna power pattern equal to zero to receive the same complete power as the antenna in use. Hence, the ratio $\frac{\Omega_{F}}{\Omega_{A}}$ is the fraction of the total power which enters the forward beam.

Similar to the definition of $F_{\text {eff }}$, the effective beam efficiency, $B_{\text {eff }}$, is defined as the factor which scales $T_{\mathrm{A}}^{\prime}$ to $T_{\mathrm{MB}}$ (Downes 1989):

$T_{\mathrm{A}}^{\prime}=B_{\mathrm{eff}} T_{\mathrm{MB}}$

Following again the notation of Kraus (1986), the effective beam efficiency is:

$B_{\text {eff }}=\frac{T_{\mathrm{A}}^{\prime}}{T_{\mathrm{MB}}}=k_{0} \frac{\Omega_{M}}{\Omega_{A}}$

where $\Omega_{M}$ is the main-beam solid angle given by the equation:

$\Omega_{M}=\iint_{\text {main beam }} P_{n}(\vartheta, \varphi) \mathrm{d} \Omega$.

Therefore, the ratio $\frac{\Omega_{M}}{\Omega_{A}}$ is the fraction of the total power which enters the main beam.

Summarizing, to scale $T_{\mathrm{A}}^{*}$ to $T_{\mathrm{MB}}$ one has to multiply $T_{\mathrm{A}}^{*}$ by the ratio of $F_{\text {eff }}$ to $B_{\text {eff }}$ (Eqs. (A3) and (A7)):

$T_{\mathrm{MB}}=\frac{F_{\mathrm{eff}}}{B_{\mathrm{eff}}} T_{\mathrm{A}}^{*}$

With the Eqs. (A4) and (A8) the ratio of $B_{\text {eff }}$ to $F_{\text {eff }}$ can be written as:

$\frac{B_{\text {eff }}}{F_{\text {eff }}}=\frac{\Omega_{M}}{\Omega_{F}}$.

In other words, this ratio indicates the fraction of the forward power which enters the main beam.

In the notation of Kutner $\&$ Ulich (1981), which was used previously for the calibration of the data for the $1.2 \mathrm{~m}$ NMWT and SMWT, $T_{\mathrm{A}}^{\prime}$, the antenna temperature of an equivalent resistor outside the atmosphere, is related to $T_{\mathrm{R}}$, the true source Rayleigh-Jeans brightness temperature (called $T_{\mathrm{B}}$ in the notation of Downes 1989), by:

$T_{\mathrm{A}}^{\prime}=\eta_{r} \frac{\Omega_{R}}{\Omega_{A}} T_{\mathrm{R}}$

where $\eta_{r}$ is the resistive loss factor of the telescope, called $k_{0}$ by Kraus (1986), defined in terms of the maximum antenna gain $G$ as:

$\eta_{r}=\frac{G}{4 \pi} \Omega_{A}$

where $\Omega_{R}$ is the solid angle subtended by the response of the source in the beam pattern, given by:

$\Omega_{R}=\iint_{\text {source }} P_{n}(\Psi-\Omega) B_{n}(\Psi) \mathrm{d} \Psi$. 
To separate the convolution of the source structure with the antenna beam pattern, Kutner \& Ulich define the radiation temperature $T_{\mathrm{R}}^{*}$ as the source intensity which is corrected for all effects except the actual coupling of the antenna diffraction pattern to the source brightness distribution. Thus, $T_{\mathrm{R}}^{*}$ is related to $T_{\mathrm{R}}$ by:

$T_{\mathrm{R}}^{*}=\eta_{c} T_{\mathrm{R}}$

where $\eta_{c}$ is the efficiency with which the antenna couples to the source. This is given by:

$\eta_{c}=\frac{\Omega_{R}}{\Omega_{D}}$

with:

$$
\Omega_{D}=\iint_{\text {diffraction area }} P_{n}(\Omega) \mathrm{d} \Omega .
$$

Thus, $\Omega_{D}$ is the diffraction-beam solid angle, and the diffraction area over which it is integrated in this equation is the area which covers the normal diffraction pattern of the antenna. Typically, this area will encompass a region within a few degrees of the telescope axis.

With this, $T_{\mathrm{A}}^{\prime}$ is related to $T_{\mathrm{R}}^{*}$ by:

$T_{\mathrm{A}}^{\prime}=\eta_{r} \frac{\Omega_{D}}{\Omega_{A}} T_{\mathrm{R}}^{*}$

Because the forward spillover and scattering arises from the sky, at sky temperature, and the rearward spillover and scattering from the ground, at ambient temperature, Kutner \& Ulich divided the spillover and scattering efficiency $\frac{\Omega_{D}}{\Omega_{A}}$ into the product of the forward $\left(\eta_{\mathrm{fss}}\right)$ and the rearward $\left(\eta_{\mathrm{rss}}\right)$ part, given by:

$$
\begin{aligned}
& \eta_{\mathrm{fss}}=\frac{\Omega_{D}}{\Omega_{F}} \\
& \eta_{\mathrm{rss}}=\frac{\Omega_{F}}{\Omega_{A}} .
\end{aligned}
$$

Defining the telescope efficiency $\eta_{l}$ and the extended source efficiency $\eta_{s}$ as:

$$
\begin{aligned}
\eta_{l} & =\eta_{r} \eta_{\mathrm{rss}} \\
\eta_{s} & =\eta_{r} \eta_{\mathrm{rss}} \eta_{\mathrm{fss}}
\end{aligned}
$$

Kutner \& Ulich obtained:

$T_{\mathrm{A}}^{\prime}=\eta_{l} T_{\mathrm{A}}^{*}$

$T_{\mathrm{A}}^{\prime}=\eta_{s} T_{\mathrm{R}}^{*}$.

Thus, $T_{\mathrm{R}}^{*}$ is related to $T_{\mathrm{A}}^{*}$ by:

$T_{\mathrm{R}}^{*}=\frac{T_{\mathrm{A}}^{*}}{\eta_{\mathrm{fss}}}$
As Kutner \& Ulich have shown, $\eta_{l}$ can be fitted by an antenna tipping procedure because the observed antenna temperature of the sky, $T_{\mathrm{A}}^{\mathrm{SKY}}$, is then given by:

$T_{\mathrm{AKY}}^{\mathrm{SKY}}=\eta_{l} T_{\mathrm{SKY}}+\left(1-\eta_{l}\right) T_{\mathrm{AMB}}$

Because an antenna tipping was regularly done at the $1.2 \mathrm{~m}$ SMWT (and NMWT) during observations $\eta_{l}$ was always monitored. Averaged over the complete observing time, it was $0.881 \pm 0.023$, thus, very stable.

Comparing the notation of Kutner \& Ulich (1981) with the notation of Downes (1989) it becomes clear that $\eta_{l}$ is $F_{\text {eff }}$ (compare Eqs. (A20), (A21), and (A23) with Eqs. (A3) and (A4)) but that $\eta_{s}$ is not $B_{\text {eff }}$ (compare Eqs. (A19), (A22), and (A24) with Eqs. (A7) and (A8)) because $\Omega_{D}$ is not equal to $\Omega_{M}$. However, as Downes (1989) pointed out, $\eta_{\text {fss }}$ is not a telescope constant, but is a variable which must be evaluated as a function of the diameter of the source to be observed. Thus, $T_{\mathrm{R}}^{*}$ is not the appropriate parameter for comparison purposes because this temperature still contains the diffraction sidelobes through the finite aperture of the main mirror as a telescope specific contribution. Therefore, observers who use the notation of Kutner \& Ulich (1981) are advised to choose $\eta_{\mathrm{fss}}$ as $\frac{B_{\text {eff }}}{F_{\text {eff }}}$ so that $T_{\mathrm{R}}^{*}$ becomes $T_{\mathrm{MB}}$.

For the 1.2 $\mathrm{m}$ Millimeter-Wave Telescopes, the calibration is described in detail by Cohen et al. (1986) for the NMWT and Bronfman et al. (1988) for the SMWT. To derive intensities which are as independent as possible of the parameters of the telescope, they both defined their "Mini" $-\eta$ — which they called the main beam efficiency as the fraction of the forward power that enters the main beam. It is the factor $T_{\mathrm{A}}^{*}$ has to be divided by to yield $T_{\mathrm{R}}$, which they define as the physical temperature of a black body that just fills the main beam. As Cohen et al. (1986) pointed out, this $T_{\mathrm{R}}$ differs from $T_{\mathrm{R}}^{*}$ as defined by Kutner \& Ulich (1981). It is defined in exactly the same way as the main-beam brightness temperature, $T_{\mathrm{MB}}$. Thus, results obtained with the $1.2 \mathrm{~m}$ Telescopes have long been in $T_{\mathrm{MB}}$ even though this was not described as such, and the "Mini" $-\eta$, which is strictly speaking the main-beamto-forward-beam efficiency, $\eta_{\mathrm{mf}}$, is given by:

$\eta_{\mathrm{mf}}=\frac{\Omega_{M}}{\Omega_{F}}=\frac{B_{\mathrm{eff}}}{F_{\mathrm{eff}}}$.

This $\eta_{\mathrm{mf}}$ was determined for the NMWT by Cohen et al. (1986) and for the SMWT by Bronfman (1986) using the theoretical radiation pattern of the feed horn, scalar diffraction theory, and the measured antenna pattern. The result was checked observationally and is the same for both telescopes:

$\eta_{\mathrm{mf}}=0.82$. 
When one applies all these corrections, one obtains the final calibration of $T_{\mathrm{MB}}$ :

$$
\begin{aligned}
F_{\mathrm{eff}} & =\eta_{l} \approx 0.88 \\
B_{\mathrm{eff}} & =\eta_{\mathrm{mf}} \eta_{l}=0.82 \eta_{l} \approx 0.72 \\
T_{\mathrm{MB}} & =\frac{T_{\mathrm{A}}^{*}}{\eta_{\mathrm{mf}}}=\frac{T_{\mathrm{A}}^{*}}{0.82} .
\end{aligned}
$$

\section{A.3. Telescope pointing}

The coordinate system of the telescope was established with roughly 40 stars, sighted through a $3 \mathrm{~cm}$ optical telescope mounted on the primary and coaligned with the radio axis because no point source can be detected with a small telescope at millimeter wavelengths in short integration times (Cohen 1977). This was done twice a year. To check if a realignment of the telescope coordinate system is required, every few days the pointing was checked in the radio by scanning through the limbs of the Sun to determine its center to within $10^{\prime \prime}$ (Cohen et al. 1986; Grabelsky et al. 1987).

During the observations, the pointing accuracy was ensured by monitoring the tracking of the telescope constantly. If the tracking error exceeded the limit of $1^{\prime}$ the integration was interrupted. Therefore, the pointing of the $1.2 \mathrm{~m}$ SMWT was always better than 1.'0 (0.11 beamwidths at the $\mathrm{C}^{18} \mathrm{O}(1-0)$ line frequency).

\section{B. The used OFF positions}

For the weighted-OFFs position switching mode (see Appendix A.1), 59 ofF positions were entered to the list in the telescope control computer. From these, the telescope control program subsequently selected the appropriate OFF positions. These positions, listed in Table 4, were verified to be free of ${ }^{12} \mathrm{CO}(1-0)$ emission stronger than $0.04 \mathrm{~K}$. Most of these are also published in Bitran (1987), Bitran et al. (1997) and Bronfman et al. (1988, 1989).

\section{References}

Altenhoff W.J., Downes D., Pauls T., Schraml J., 1978, A\&AS 35,23

Armstrong J.T., Barrett A.H., 1985, ApJS 57, 535

Bally J., Stark A.A., Wilson R.W., Henkel C., 1987, ApJS 65, 13

Bally J., Stark A.A., Wilson R.W., Henkel C., 1988, ApJ 324, 223

Bania T.M., 1977, ApJ 216, 381

Bania T.M., 1980, ApJ 242, 95

Bania T.M., 1986, ApJ 308, 868

Bitran M.E., 1987, Ph.D. thesis, University of Florida

Bitran M.E., Alvarez H., Bronfman L., May J., Thaddeus P., 1997, A\&AS 124, 1

Bronfman L., 1986, Ph.D. thesis, Columbia University New York
Table 4. The used ofF positions

\begin{tabular}{cc}
$\mathbf{l}\left(^{\circ}\right)$ & $\mathbf{b}\left(^{\circ}\right)$ \\
\hline 338.50 & +7.70 \\
339.00 & +5.00 \\
340.00 & +2.00 \\
342.70 & -6.00 \\
342.90 & +3.70 \\
344.00 & -5.00 \\
344.30 & +5.80 \\
345.00 & -5.00 \\
346.00 & -5.00 \\
346.00 & -3.00 \\
347.00 & +3.00 \\
348.00 & -5.00 \\
348.00 & +4.00 \\
349.00 & -6.00 \\
350.00 & -7.50 \\
351.00 & -6.00 \\
352.00 & -8.50 \\
352.00 & -5.00 \\
353.00 & -5.00 \\
353.00 & +4.00 \\
\hline
\end{tabular}

\begin{tabular}{rr}
$\mathbf{l}\left(^{\circ}\right)$ & $\mathbf{b}\left(^{\circ}\right)$ \\
\hline 353.50 & +6.00 \\
354.00 & +5.00 \\
355.00 & -5.00 \\
355.00 & +5.00 \\
356.04 & -5.23 \\
357.00 & -8.00 \\
357.00 & -5.00 \\
357.00 & +3.00 \\
358.00 & -7.00 \\
358.00 & +8.00 \\
359.80 & -5.75 \\
0.00 & -7.00 \\
0.70 & -3.30 \\
1.00 & -8.00 \\
1.00 & -3.90 \\
1.00 & +6.00 \\
1.90 & -5.35 \\
2.00 & -7.00 \\
2.50 & -5.00 \\
3.80 & -4.80 \\
\hline
\end{tabular}

\begin{tabular}{rr}
$\mathbf{l}\left(^{\circ}\right)$ & $\mathbf{b}\left(^{\circ}\right)$ \\
\hline 4.00 & -6.50 \\
4.75 & +2.50 \\
5.00 & -4.00 \\
5.00 & +5.00 \\
5.50 & -7.50 \\
7.00 & -5.00 \\
8.00 & -5.00 \\
8.50 & +2.75 \\
9.00 & +5.00 \\
9.00 & +7.50 \\
10.00 & +5.00 \\
10.00 & +10.25 \\
11.00 & -4.00 \\
11.00 & +8.00 \\
12.00 & -5.00 \\
12.00 & +5.00 \\
13.00 & +7.50 \\
14.00 & -2.50 \\
15.00 & -5.00 \\
\hline
\end{tabular}

Bronfman L., Cohen R.S., Alvarez H., May J., Thaddeus P., 1988, ApJ 324, 248

Bronfman L., Alvarez H., Cohen R.S., Thaddeus P., 1989, ApJS 71, 481

Churchwell E., Wood D., Myers P.C., Myers R.V., 1986, ApJ 305,405

Cohen R.J., Few R.W., 1976, MNRAS 176, 495

Cohen R.J., Dent W.R.F., 1983, OH in the Centre of the Galaxy. In: Burton W.B., Israel F.P. (eds.), Surveys of the Southern Galaxy. Proceeding of a workshop at the Leiden Observatory, The Netherlands, p. 159

Cohen R.S., 1977, Ph.D. thesis, Columbia University New York

Cohen R.S., 1983, Columbia University Southern Hemisphere Millimeter-Wave Survey Telescope. In: Burton W.B., Israel F.P. (eds.), Surveys of the Southern Galaxy. Proceeding of a workshop at the Leiden Observatory, The Netherlands, p. 265

Cohen R.S., Dame T.M., Thaddeus P., 1986, ApJS 60, 695

Cummins S., Linke R.A., Thaddeus P., 1986, ApJS 60, 819

Dame T.M., 1992, Notes to Observers Manual, HarvardSmithsonian Center for Astrophysics, Cambridge (Mass.), Internal Report

Dame T.M., Ungerechts H., Cohen R.S., et al., 1987, ApJ 322, 706

Dahmen G., 1995, Dissertation, Universität Bonn

Dahmen G., Henkel C., Hüttemeister S., et al., 1993, Astr. Ges. Abstr. Ser. 9, 162

Dahmen G., Hüttemeister S., Wilson T.L., et al., 1996, The Molecular Gas in the Galactic Center Region based on $\mathrm{C}^{18} \mathrm{O}$ Measurements. In: Gredel R. (ed.), The Galactic Center, Proceedings of the $4^{\text {th }}$ ESO/CTIO Workshop, ASP Conf. Ser. 102, 54 
Dahmen G., Hüttemeister S., Wilson T.L., 1997 (Paper II) (submitted to A\&A)

Downes D., Maxwell A., 1966, ApJ 146, 653

Downes D., Goss W.M., Schwarz U.J., Wouterloot J.G.A., 1978, A\&AS 35, 1

Downes D., 1989, Radio Astronomy Techniques. In: Appenzeller I., Habing H.J., Léna P. (eds.), Evolution of Galaxies Astronomical Observations, Lecture Notes in Physics 333. Springer, Berlin - Heidelberg, p. 351

Grabelsky D.A., Cohen R.S., Bronfman L., Thaddeus P., May J., 1987, ApJ 315, 122

Handa T., Sofue Y., Nakai N., Hirabayashi H., Inoue M., 1987, PASJ 39, 709

Heiligman G.M., 1987, ApJ 314, 747

Hoffmann W.F., Frederick C.L., Emery R.J., 1971, ApJ 164, L23

Huntress W.T., Mitchell G.F., 1979, ApJ 231, 456

Hüttemeister S., 1993, Dissertation, Universität Bonn

Iglesias E., 1977, ApJ 218, 697

Jackson J.M., Armstrong J.T., Barrett A.H., 1984, ApJ 280, 608

Jackson J.M., Heyer M.H., Paglione T.A.D., Bolatto A.D., 1996, ApJ 456, L91

Kaifu N., Kato T., Iguchi T., 1972, Nat. Phys. Sci. 238, 105

Kraus J.D., 1986, Radio Astronomy, 2nd ed. McGraw-Hill Book Company, New York

Kutner M.L., 1978, Astrophys. Lett. 19, 81

Kutner M.L., Ulich B.L., 1981, ApJ 250, 341
Lequeux J., 1962, Ann. d'Astrophys. 25, 221

Linhart A., 1994, Diplomarbeit, Universität Bonn

Liszt H.S., Burton W.B., 1978, ApJ 226, 790

Lovas F.J., 1992, J. Phys. Chem. Ref. Data 21, 181

Mauersberger R., 1994, Habilitationsschrift, Universität Bonn

Odenwald S.F., Fazio G.G., 1984, ApJ 283, 601

Oka T., Hasegawa T., Handa T., Hayashi M., Sakamoto S., 1996, ApJ 460, 334

Palmer E.S., 1984, 256-Channel, 0.1 MHz/Channel Multifilter Spectrometer, NASA Goddard Institute for Space Studies, Internal Report

Penzias A.A., Burrus C.A., 1973, ARA\&A 11, 51

Radford S.J.E., Downes D., Solomon P.M., Barrett J., Sage L.J., 1996, AJ 111, 1021

Reich W., Fürst E., Steffen P., Reif K., Haslam C.G.T., 1984, A\&AS 58, 197

Rougoor G.W., Oort J.H., 1960, Proc. Nat. Acad. Sci. 46, 1

Scoville N.Z., 1972, ApJ 175, L127

Snyder L.E., Buhl D., 1972, ApJ 177, 619

Uchida K.I., Morris M., Bally J., Pand M., 1997 (in preparation)

Ulich B.L., Haas R.W., 1976, ApJS 30, 247

Whiteoak J.B., Gardner F.F., 1979, MNRAS 188, 445

Winnewisser G., Hocking W.H., Gerry M.C.L., 1976, J. Phys. Chem. Ref. Data 5, 79

Zylka R., Güsten R., Henkel C., Batrla W., 1992, A\&AS 96, 515 